\title{
Abstracts of the XXVIII Scientific Conference of the SEÑ and the VI Young Researchers Meeting Spanish Nutrition Society (SEÑ)
}

Soria, Spain, June 20-22, 2019

\section{Abstracts}

Guest Editors

Marcela González-Gross, Madrid

J. Alfredo Martínez-Hernández, Pamplona

María Puy Portillo Baquedano, Vitoria

Alfredo Fernández-Quintela, Vitoria

Fermin Milagro Yoldi, Pamplona 
S. Karger

Medical and Scientific Publishers Basel · Freiburg · Paris · London New York · Chennai · New Delhi Bangkok · Beijing · Shanghai · Tokyo Kuala Lumpur · Singapore · Sydney
Disclaimer

The statements, opinions and data contained in this publication are solely those of the individual authors and contributors and not of the publisher and the editor(s). The appearance of advertisements in the journal is not a warranty, endorsement, or approval of the products or services advertised or of their effectiveness, quality or safety. The publisher and the editor(s) disclaim responsibility for any injury to persons or property resulting from any ideas, methods, instructions or products referred to in the content or advertisements.

Drug Dosage

The authors and the publisher have exerted every effort to ensure that drug selection and dosage set forth in this text are in accord with current recommendations and practice at the time of publication. However, in view of ongoing research, changes in government regulations, and the constant flow of information relating to drug therapy and drug reactions, the reader is urged to check the package insert for each drug for any change in indications and dosage and for added warnings and precautions. This is particularly important when the recommended agent is a new and/or infrequently employed drug.
All rights reserved.

No part of this publication may be translated into other languages, reproduced or utilized in any form or by any means, electronic or mechanical, including photocopying, recording microcopying, or by any information storage and retrieval system, without permission in writing from the publisher or system, without permission in writing from the publisher or,
in the case of photocopying, direct payment of a specified fee to the Copyright Clearance Center (see 'General Information').

(C) Copyright 2019 by S. Karger AG

P.O. Box, CH-4009 Basel (Switzerland)

e-ISBN 978-3-318-06581-7

\section{KARGER}




\section{CONFERENCES}

1

\section{The polyhedron of the Mediterranean diet}

\section{Juan Manuel Ruiz Liso}

Spanish Representant of Mediterraen Diet at UNESCO

Unfortunately, something as important as Mediterranean Diet(MD) has not affected the population, conceptually, in an integral way, because its two words of origin imply misunderstandings. And this is because we have forgotten the etymology of them, precise and concise -even- in the Dictionary of Sebastian de Covarrubias of 1610:Diet comes from the Greek <<Diaita>> and its translation is "lifestyle" with all the positive connotations that it entails. Mediterranean does not mean riparian of this sea. It is, according to Covarrubias, <<what is in the middle of the earth separated from the sea. And from here it was called the Mediterranean Sea, because it is far from the Ocean Sea, and it crosses through the middle of the earth, dividing the Africa of Europe ... (sic) >>. It is, then, the interior Spain, the truly Mediterranean and more than its coastal provinces. Therefore we must move away from the dietetic and gastronomic vision that coastal products are the "Mediterranean". The MD is also an album of traditions, of many generations, in which sayings and songs have great value. In this context, women are the best transmitters of this way of life.

Fulfilling food and dietary patterns exclusively is not MD. This is a polyeder of different faces in which as important as food, although with different weight, are physical exercise and sport, the healthy environment, sustainability, cross-sectional education in schools, communication, optimism, siesta, and other elements that we will see in our exhibition, pondering fresh and local products.

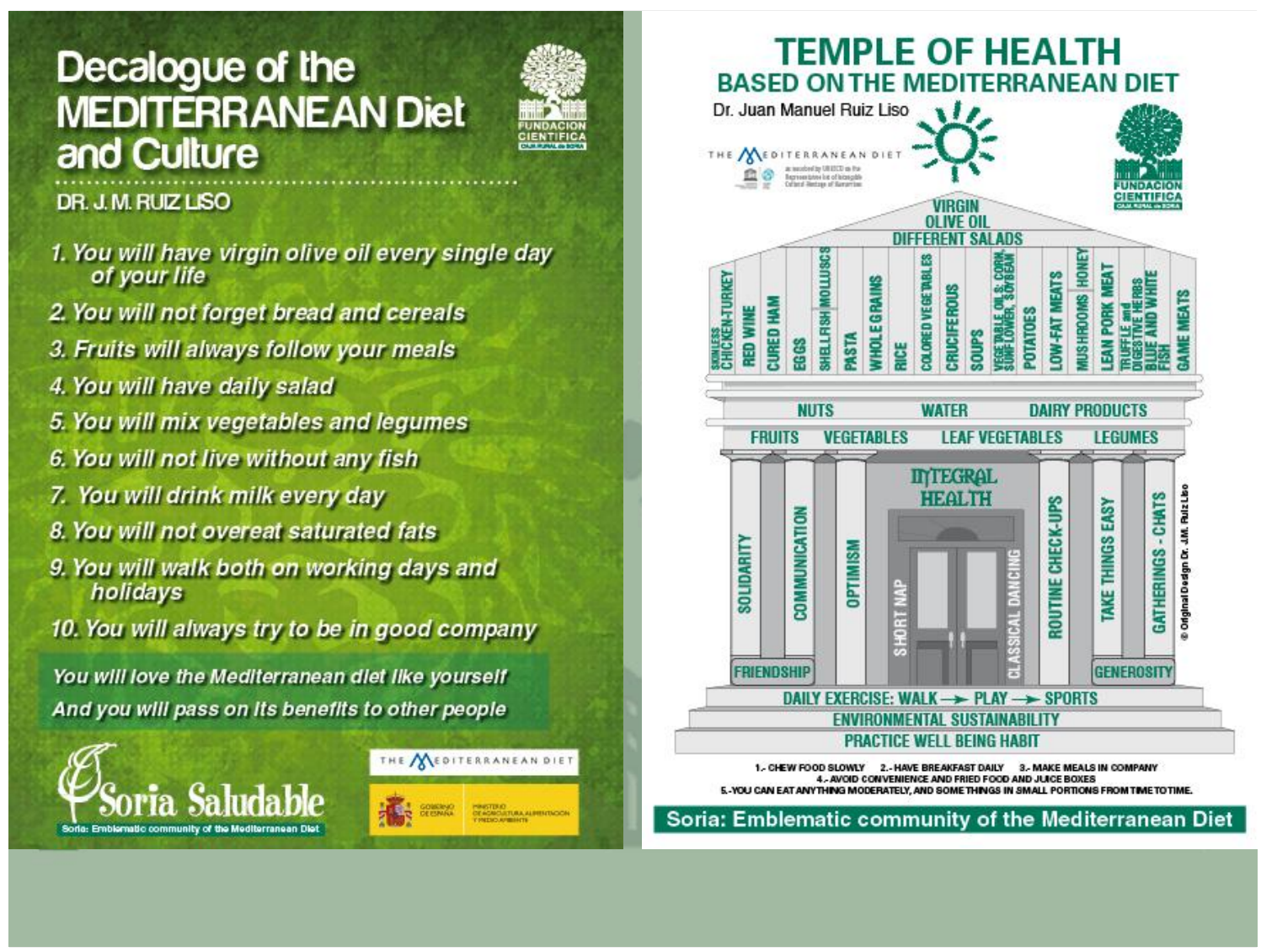


Monitoring urban wastewater: an innovative approach for assessing community health

\section{Félix Javier Hernández Hernández}

Full Professor Analytical Chemistry, head of Analytical Chemistry and Public Health group (Institute for Pesticides and Water), and of the GLP-certified Pesticide Residue Analysis Laboratory, UnivJaume I, Castellón, Spain.

Urban wastewater contains a complex mixture of chemical substances with a wide range of human excretion products of external or internal bodily origin resulting from exposure to xenobiotics (e.g. illicit drugs, food or environmental toxicants and infectious agents) and internal processes (e.g. specific disease or exposurelinked proteins, genes and metabolites). The quantitative measurement of specific endo- and exogenous residues (biomarkers) in wastewater can provide valuable evidence of the quantity and type of xenobiotic chemical, biological or physical agents to which the surveyed population is exposed. Thus, theanalysis of WWoffersanonymized, but comprehensive and objective information on the health status of urban inhabitants and surrounding environments in real time. Wastewater-Based Epidemiology (WBE) in a cutting-edge approach able to extract epidemiological information from urban WW. WBE is currently used to report on worldwide illicit drug use trends, providing valuable data to the European Monitoring Centre for Drugs \& Drug Addiction. WBE has been also applied to verify wastewater fingerprints of alcohol, tobacco and counterfeit medicines.

WBE can be applied to monitoring WW for chemicals that serve as indicators of the collective status of human health (or stress/disease) or any other facet relevant to assess time-trends in community-wide health.Of particular interest is the monitoring of a broad suite of endogenous biomarkers of key physiologic processes (as opposed to xenobiotics or their metabolites). This approach can be consideredanalogous to a hypothetical community-wide collective clinical urinalysisor to a hypothetical en massehuman biomonitoring program. In this case, the target analytes are not xenobiotics, but rather biomarkers of endogenous biochemical processes.

In this presentation, the WBE fundamentals are briefly discussed focused on the mostwidely applied area, i.e. estimation of drugs use. Later, the criteria for selecting suitablebiomarkers are discussed, and the potential of this approach for investigation of dietary markers in wastewater, as indirect indicators of health/wellness, is commented. Examples of candidate markers for consumption of nutritious foods includealkylresorcinols and polyphenols, while candidates for consumption of potentially harmful foods include: metabolites of ethyl alcohol, of the mycotoxin DON, or methyl histidine.

C. Ort, et al. (2014). Addiction 109: 1338-1352

E. Gracia-Lor,et al. (2017).Environ Int99: 131-150

http://www.emcdda.europa.eu/news/2016/12/latest-data-reveal-drug-taking-habits-in-over-50-europeancities\#news

C.G. Daughton(2018). Sci Total Environ 619-620: 748-764

\section{What contribution do fatty acids make to cardiovascular risk ?}

\section{Jacques Delarue}

Professor of Nutrition. Department of Nutritional Sciences and Human Nutrition Laboratory of Brest University Hospital (France).

The Seven Countries Study demonstrated that there was 10 times higher incidence of cardiovascular mortality in East Finland as compared to Crete. This was attributed at this time to hypercholesterolemia induced by high saturated fat diet consumed in Finland in 70's. Very recently, the protective effect of a Mediterranean diet associated to a high intake of olive oil or of nuts decreased by $30 \%$ the primary CV risk in people at high risk (Predimed study). Between these times, progress has been made to better delineate the contribution of different fat types to CV risk. It has been clearly demonstrated that a high consumption of trans fat and of saturated fat increases the CV risk. Conversely, polyunsaturated fat (PUFAs) decrease CV risk. Long chain omega 3 fatty acids decrease primary and secondary CV risk, which is far less controversial thanduring the past recent years since new randomized studies demonstrate or confirm their protective effects. It is very important to consider what should be substituted to saturated fat in order to decrease the CV risk. Refined carbohydrates (CHO) when substituted for saturated fat increase CV risk whereas PUFAs decrease it. Whole grains when substituted to saturated fat also decrease CV risk. Thus, trans fat must be eliminated from industrial processes, whole fat should not exceed $30 \%$ of total energy intake and saturated 
fat should be less that $10 \%$ of TEI. An amount of $\geq 1 \mathrm{~g} / \mathrm{d} 1 \mathrm{~g}$ of EPA + DHA is required for secondary CV prevention and of $\geq 500 \mathrm{mg} / \mathrm{d}$ for primary prevention.

4

PalaeoChef. From the termites to the plankton

Ana Mateos Cachorro; Jesús Rodríguez Méndez

Centro Nacional de Investigación sobre la Evolución Humana (CENIEH), Burgos

Introduction. Humans are primates that changed their food habits during millions of years of evolution. Our species, Homo sapiens, has a broad and diversified diet that reflects the biological features and behavioural acquisitions of our most distant ancestors. In essence, feeding is one of the keys to understand our past, present and future as a human species. Many of our adaptive successes may be attributed to the metabolic and physiological gear modelled by evolution along several millions of years, but also to our abilities obtaining, processing and consuming foods.

Objectives. To review the main adaptive events occurred during more than 3 millions years of human evolution related to brain expansion, anatomical and physiological changes, environmental restrictions and the technological and cultural system in different hominin species. In addition, the metabolic constraints of plio-pleistocene hominins during different physical activities are evaluated, to test hominin energetic efficiency.

Conclusions. For millions of years, the species of the human lineage discovered the nutritional and healthy advantages of a variety of food resources and of food processing and preparation. Almost everything that is edible for our species was incorporated into the human diet, by different cultures. The challenge for humans will be maintain the energy balance of our physiological engine while not compromising the survival and viability of our species on Earth. The pleasure of eating in a diversified way, may contribute to sustain our species on the planet nowadays as it did millions of years ago in the African savannas.

Conflict of interest. Authors declare no conflict of interest.

Acknowledgements. METHOD International Focus Group (1604F) founded by the INQUA Humans and Biosphere Commission (HabCom).

Key words. Hominins. Human diet. Energy balance.

5

\section{Recent genetic variability in hominids and itsrelevance in the current distribution of macronutrients}

\section{Jesús Rodríguez Huertas}

Catedrático de Fisiología de la Universidad de Granada.

The distribution of macronutrient intakes has been questioned in the last few years, reducing the carbohydrate intake. The ketogenic and paleolithic diets are spreading disturbingly andleading to a decrease in the health of the population.

The paleolithic diet is based on the premise that humans are genetically adapted to the diet of their paleolithic ancestors. Therefore, these diets include foods supposedly available before the Neolithic revolution, mainly based on meat, fish, fruits, vegetables, nuts and roots, and exclude grains, legumes, dairy products, salt, refined sugars and processed oils.

However, mutations and/or polymorphisms were selected during the Holocene, at the Neolithic stage. This selection allowed us the intake of foods that have played akey role in the development of our species. During that time, humans learned to raise crops and livestock. Consequently, humans were no longer dependent on hunting, fishing, and gathering wild plants. The lactase persistence, the variation in the number of copies ofthe salivary amylase gene or the gluten tolerance allowed us to incorporate foods that made us more human, such as milk, starches and cereals.

Some ethnic groups, such asInuit, underwentdifferentadaptations and these groupsare used as an argument in defence of ketogenic diets.However, the Inuit diet, rich in protein and omega-3 polyunsaturated fatty acids (PUFAs), is a consequence of a genome adaptation at several loci related with fatty acid desaturases. The selected alleles are associated with multiple metabolic and anthropometric phenotypes.

In the light of the foregoing,very relevantgenetic adaptations were produced duringthe Neolithic period, which determinedthe greater energy intakefrom carbohydrates in our modern diet. 


\title{
Dieta celtibérica
}

\author{
Alfredo Jimeno Martínez \\ Universidad Complutense de Madrid, Madrid
}

En la necrópolis de Numancia los análisis de los restos de los productos de la molienda incrustados en los molinos, se ha podido concretar en la molienda de bellota, trigo y cebada. Los componentes alimenticios muestran diferencia de sexos tanto en los ajuares como en la dieta alimenticia. Se conserva mayor consumo de cereales, vegetales verdes, legumbres y carne asociados a los enterramientos con armas. Por el contrario en las tumbas que no contienen armas, se observa una dieta más rica en frutos secos, bayas y tubérculos asociados con adornos.

\section{Polyphenols and obesity}

\section{María del Puy Portillo Baquedano}

Faculty of Pharmacy. University of the Basque Country Centro de Investigación Biomédica en Red (CIBEROBN)

Background: Overweight and obesity represent a public health problem due to their high prevalence, not only in adults but also in children. Obesity induces a great number of co-morbidities such as insulin resistance and diabetes, hypertension, dyslipidemia and fatty liver among others. Usually, overweight and obesity are treated by using hypocaloric diets. Nevertheless, the adherence to this dietetic intervention is low very often and consequently the effectiveness of the treatment is lower than expected. In this context scientific community is looking for new molecules, naturally present in foodstuffs that can be useful for overweight and obesity prevention and management as complementary tools. Objectives: The aim of this study is to review the results provided in the literature concerning the effects of polyphenols as anti-obesity molecules. Results: A great number of studies have shown that several polyphenols are able to reduce body fat accumulation. On the most studied phenolic compounds is resveratrol (3,4',5-trihidroxistilbeno), a stilbene present in grapes and wine, peanuts and berries. Numerous preclinical studies carried out in cultured cell and animals models have showed that resveratrol reduces triglyceride accumulation in adipocytes. The vast majority of them have used an experimental design were this molecule is administered at the same time than an obesogenic diet. Although very few studies have addressed the effects of resveratrol when administered alone or in combination with energy restriction, data reported show that, under these experimental conditions, resveratrol is not effective. Conclusion: Resveratrol seems to be useful to prevent obesity but not to treat this pathology or to improve effects of an energy restricted diet.

\section{Food bioactive amines and related disorders}

\section{Carmen Vidal Carou}

Departament de Nutrició, Ciències de l'Alimentació i Gastronomia, Facultat de Farmàcia i Ciències de l'Alimentació, Universitat de Barcelona, Institut de Recerca en Nutrició i Seguretat Alimentària (INSA·UB), Universitat de Barcelona,

Histamine intolerance (HIT) mainly arises from the failure of diamine oxidase (DAO) enzyme to metabolize histamine from food at intestinal level and is characterized by a wide range of gastrointestinal nonspecific symptoms and extra-intestinal symptoms, such as headache, atopic dermatitis and eczema. Current treatment for HIT consists on the follow-up of low-histamine diets based on the exclusion of foods that patients relate to the onset of symptoms. In addition, and considering the key role of DAO enzyme in the breakdown of dietary histamine, orally administered DAO supplements have been proposed as a strategy to enhance histamine degradation and improve the quality of life of intolerant individuals undergoing tough dietary restrictions.

This work aims to review the state-of-the-art in the efficacy of low-histamine diets and DAO supplementation and to share recent developments on new strategies for the diagnostic and control of HIT. 
Several clinical studies reported in the literature have unanimously demonstrated the efficacy of lowhistamine diets, with a percentage of patients showing an improvement of symptoms ranging from $33 \%$ to $100 \%$. In addition, different success rates have also been reported by the use of exogenous DAO supplementation, although clinical studies dealing with this topic are still scarce and small-sized.

Histamine degradation capability of commercial DAO supplements has been confirmed in vitro, with great variability among the different products available in the market. Moreover, a screening has been performed to select new potential sources of this enzymatic activity, with interesting leads with several plant-origin matrixes and certain strains of probiotic bacteria. Finally, a new approach for the diagnosis of HIT has been developed based on the determination of the urinary metabolomic profile of histamine.

Due to the growing awareness of HIT, it is important to promote further research to unfold new and stronger evidence on this disorder. Efforts need to be devoted in designing further clinical studies to continue stablishing the efficacy of the currently proposed treatment strategies.

\section{Proteins and Sarcopenia}

\section{Alfredo Córdova Martínez}

Dept. of Biochemistry, Molecular Biology and Physiolog. Faculty of Health Sciences - SORIA. University of Valladolid

The current definitions of sarcopenia, collected by Dennison et al. (2017), include both a loss of muscle mass and a decrease in muscle function associated with age. Sarcopenia, in general, has a prevalence of 5-13\% in people of $60-70$ years old and $11-50 \%$ in people over 80 years old. In addition, Cruz-Jentoft et al. (2014), have observed a prevalence between 1 and $29 \%$ in elderly people living in their homes, and between 14 and $68 \%$ in institutionalized elderly.

Many risk factors associated with sarcopenia. In a recent review (Marzetti et al., 2017), they indicate that diseases that involve chronic inflammation and metabolic imbalances, as well as nutritional deficiencies, especially in terms of energy and proteins, have a big impact on muscle health, promoting the appearance of sarcopenia. Two categories of sarcopenia are distinguished: a) primary related to age (there is no other obvious cause except aging) and b) secondary, when there are one or several other obvious causes. In this case, one of the fundamental factors is the consequence of an insufficient dietary intake of energy and / or proteins.

With respect the prevention and treatment of sarcopenia, Cruz-Jentoft et al. (2014), showed that nutritional interventions and those based on physical exercise improve muscle strength and physical performance. Steffl (2017) in a meta-analysis concluded that physical activity is determinant independent of the type of exercise that is carried out. On the other hand, the evidence suggests that the maintenance of adequate protein intake attenuates muscle atrophy due to disuse (Wall and van Loon, 2013). There are studies (Martone et al., 2017; Deutz et al., 2014; Bauer et al., 2013), suggesting that protein recommendations in elderly people are very low $(0.8 \mathrm{~g} / \mathrm{kg} /$ day) proposing that an optimal intake would be $1-1.2 \mathrm{~g} / \mathrm{kg} /$ day, and even 1.2 to $1.5 \mathrm{~g} / \mathrm{kg} /$ day in the elderly with acute or chronic diseases. In addition, Paddon-Jones and Leidy (2014). Have indicated that not only the total amount of protein is important, but also the amount ingested in each meal, suggesting a contribution of approximately $30 \mathrm{~g}$ of protein/daily food.

Key words: Sarcopenia, nutrition, proteins, physical exercise.

\section{La vitamina $D$, más allá del sistema óseo}

\section{Pilar Riobó Serván}

\section{Fundación Jiménez Diaz. Quiron salud. Madrid}

Vitamin D is currently one of the hottest topics in medicine. Over the last decades, scientific studies have demonstrated that the pandemic global vitamin D deficiency not only has a negative impact on demineralization of bones, but also facilitates development and progression of multiple diseases of civilization, including cardiovascular diseases, diabetes, autoimmune disease, and cancer; these have denominated the pleiotropic effects of vitamin D.It is well known that vitamin D inhibits proliferation and induces differentiation of the cells, and is essential for maturation of immune cells. Lymphocytes, neutrophils, monocytes, and dendritic cells express VDR and also activate circulating 25(OH)D3 through hydroxylation by CYP27B1. It has been recently suggested that vitamin D could be useful in the prevention 
and treatment of autoimmune diseases such as multiple sclerosis, type 1 diabetes mellitus, rheumatoid arthritis, or systemic lupus erythematous. Sessional respiratory infections, such as influenza, have been associated to vitamin D deficiency. A low level of vitamin D is associated with an increased risk of any type of cancer and a decrease survival rate. In clinical studies, vitamin D supplementation is associated with an increase in overall survival and lower risk of relapse Vitamin D intake and blood 25(OH)D levels were inversely associated with the risk of colorectal cancer. But in the Vitamin D Assessment (ViDA) randomized clinical trial that included 5108 community adults in New Zealand, the cumulative incidence of cancer for a median follow-up period of 3.3 years was $6.5 \%$ among participants receiving 100000 IU of vitamin D3 monthly and $6.4 \%$ among participants receiving placebo. So, monthly high-dose vitamin D supplementation may not be associated with cancer prevention and should not be used for this purpose.Even more, recent studies have shown that vitamin D can be used in adjuvant radio-therapy. Observational studies support an association between a low blood 25-hydroxyvitamin D level and the risk of type 2 diabetes. But among persons at high risk for type 2 diabetes not selected for vitamin D insufficiency, vitamin D3 supplementation at a dose of 4000 IU per day did not result in a significantly lower risk of diabetes than placebo.

The involvement of vitamin D in the regulation of the cardiovascular system and its possible effect on hypertension is currently under investigation. Also, vitamin D deficiency has been associated with several neurological diseases (multiple sclerosis, stroke, Alzheimer's and Parkinson's diseases), as the vitamin D receptor is expressed in several brain structures and regulates the expression of neurotrophinsand neurotransmitters. If confirmed in clinical trials, vitamin D supplementation may reduce long-term disability.

\section{Critical nutrients in sports nutrition}

\section{Marcela González Gross}

ImFINE Research Group. Department of Health and Human Performance. Faculty of Physical Activity and Sport Sciencies-INEF. Universidad Politécnica de Madrid. Spain.

CIBEROBN, Biomedical Research Networking Center for Physiopathology of Obesity and Nutrition, Carlos III Health Institute, Madrid, Spain.

Sports nutrition is evolving fast in the last years. First meant only for elite athletes, the increasing number of people who is practicing exercise on a regular basis has broaden the spectrum also on amateur athletes. The American College of Sports Nutrition in the last position stand indicates clearly that recommendations are for "active adults and competitive athletes".

Nutrient deficiencies that are highly prevalent in the general population also affect active subjects, as vitamin D, iron, calcium, folate. Low intake of antioxidants have been identified in many studies, but increased intake beyond needs can be detrimental for performance. Vegetarian and vegan diets are gaining in popularity among both professional and recreational athletes, which increases deficiency risk of critical nutrients and makes supplementation essential. Protein and amino acid intake has shifted from total daily recommendations to distributed intakes during the day, with a special focus on pre and post-training intake. Water is a critical nutrient both during the day and before, during and after exercise, together with electrolytes and glucose. Interestingly, most athletes, irrespective of their sport modality, eat fewer carbohydrates than recommended. This has risen the doubt if recommendations are adequate or not.Recommendations on sugar intake restriction meant for the general (sedentary) population may be are affecting also active subjects' carbohydrates intake. Complexity of sports nutrition is even higher than general nutrition, as not only biological and genetic factors have to be taken into account. Sport modality, training schedule, rules, and environment, among others,determine nutrient needs. In the ageing master athlete, adequate nutrient intake is essential to preserve joint health, bone health, cardiovascular health, cognitive function, etc. The optimal combination of adequate nutrient intake and training programme is a challenge that research is facing currently. 


\section{Nutrition in sports performed in hypoxic conditions}

\section{Enrique Roche Collado}

Institute of Bioengineering and Department of Applied Biology-Nutrition, University Miguel Hernandez, Alicante Institute for Health and Biomedical Research (ISABIAL Foundation), Alicante, Spain. CIBER, Fisiopatología de la Obesidad y la Nutrición, CIBERobn, Instituto de Salud Carlos III (CB12/03/30038). Spain.

A massive expansion of mountain tourism and the practice of sports at extreme altitude have been observed in the last decades, despite the extreme hazardous conditions of this environment.These new forms of sports and recreation represent an increase in altitude-associated disorders and related accidents. The exposure to the hypoxic conditions that represent high-mountain expeditions, is associated to an increased production reactive oxygen species and oxidative stress. However, there is little information regarding prevention of altitude-related disorders to preparea trekking at extreme altitudes as well as strategies for recovery after this type of expeditions. In this context, acclimatization strategies have been shown to be the best solutions to avoid altitude-related disorders in order to prepare a mountain expedition. Therefore, we have designed a protocol that combines high intensity intermittent endurance-resistance training in normobaric hypoxia with mountain trekking at mid altitude together with a dietetic plan rich in proteins and supplemented with leucine (to serve as guconeogenic substrate), iron (to facilitate hemo synthesis) and vitamin E (to reinforce antioxidant defenses).The application of this training-dietetic protocol reduced significantly the incidence of acute mountain sickness. Regarding thereturn to moderate altitude after the expedition, we have observed that oxidative stress persists resulting in an incomplete recovery. In particular, neutrophils displayed high levels of the oxidative stress marker malondialdehyde, together with low catalase activity and increased plasmatic protein carbonyls. This suggests that the intake of antioxidants might continue several days after a mountain expedition. In conclusion, the presented data reinforce the role of antioxidants in the prevention, adaptation and recovery of altitude-related oxidative stress.

\section{Advances in sports nutrigemonics: impact of supplementation on performance in athletes}

\section{Diego Fernández-Lázaro}

Faculty of Health Sciences - SORIA. University of Valladolid

Sport and exercise performance are significantly influenced by nutrition, yet individuals respond differently to the same foods, nutrients and supplements consumed. For this reason, the importance of a personalized sports nutrition plan, these strategies encompass overall dietary patterns, macronutrient ratios, micronutrient requirements, eating behaviors and the judicious use of supplements and ergogenic aids. In this sense,

the genetics play an influential role in determining how an athlete responds to foods and supplements, because the genetic variants affect the way we absorb, metabolize, utilize and excrete nutrients, and genediet interactions that affect metabolic pathways relevant to health and performance are now widely recognized. Personal genetic testing can provide information that will guide the practical application, for personalized nutrition, of the scientific knowledge on health and performance. The objective of this conference is to examine the scientific evidence on specific and most used supplements on athletes, whereby genetic variants appear to modify individual responses related to athlete health and athletic performance. Although, some have not been studied in athletes exclusively, they have been carried out in healthy individuals. The nutrigenomics approach to sport nutrition. An athlete is exposed to a food, beverage, nutrient or bioactive. A genetic variant such as a single nucleotide polymorphism (SNP) associated with that exposure modifiers the individual's requirement for or response to that exposure. Their unique response depends on their version of the gene or genotype impacting response to various micro- and supplements such as caffeine, vitamin A, Iron, Folate, and Vitamin B12 (Sport Anemia-Related), vitamin C and vitamin D. In this sense, the genetic variants that modify the association between various dietary factors and performance-related outcomes are showed in figure 1 . Therefore, the current science nutrigenomic linking genetic variation to nutritional or supplemental needs with a focus on sport performance and more specifically, personalized nutrition pursuits aim to develop more comprehensive and dynamic supplement recommendations based on shifting,interacting parameters in an athlete's intrinsic and extrinsic sport factorsenvironment throughout their athletic career. 


\section{Healthy lifestyles for nutritional well-being}

\section{Francisco José Tinahones Madueño}

Departamento de Medicina Interna, Instituto de Investigación Biomédica de Málaga (IBIMA). Hospital Universitario Regional de Málaga, España. CIBER Fisiopatología de la Obesidad y la Nutrición. Instituto de Salud Carlos III. Madrid, España. Departamento de Endocrinología y Nutrición, Hospital Universitario Virgen de la Victoria, Universidad de Málaga. Instituto de Investigación Biomédica de Málaga (IBIMA), Málaga, España.

Obesity is already recognized as a worldwide pandemic, affecting more than 600 million people and, according to the World Health Organization, increasing to a ratio of 30 million cases per year. Combating it is one of the greatest challenges of the 21st century. It is shown that there are different pathologies such as cardiovascular disease, diabetes mellitus or cancer associated with excess weight that leads to a higher incidence of mortality in this population. It is well established that proneness to obesity depends on a complex interplay between numerous factors that are subjected to both genetic and environmental influences. Most of the relevant literature emphasizes that obesity is explained by suboptimal macronutrient composition of the diet and insufficient physical activity. Current guidelines for obesity management recommend weight loss for all obese individuals and overweight individuals with comorbidities. To achieve this weight loss there are several lines of action, therapeutic measures and/or more recommended, changes in the lifestyle of the subject, that is, hypocaloric diet and increased physical activity. Adiposity and lifestyle behaviours both have important function in predicting metabolic status.There are enough findings demonstrating the beneficial health effects when is introduces lifestyle-based modifications to resolve metabolic risk factors in individuals with weight excess, particularly those with significant metabolic alterations. With healthy lifestyle, even slightly weight loss ( $<5 \%$ of initial body weight $)$ and 30 min of daily physical activity, significantly improves the metabolic risk factors.

\section{Food and Societyin Spain of the21st Century: Food Decision and Sustainability}

\section{Gregorio Varela Moreiras}

Faculty of Pharmacy. University San Pablo-CEU. Madrid. Spain.

There is a growing body of recent evidence linking different social aspects of food (shopping location, culinary skills, sociability, but also sustainability and diet decisión-making) with healthier dietary choices and better adherence to nutritional guidelines. In fact, the most recent food pyramids, such as the Mediterranean Diet Pyramid and the one designed for the Spanish population, include these items as the first step to achieve a better and healthy diet. Paradoxicallly, however, there is a lack of information in Spain, whereas significant social changes (e.g. incorporation of women to work, urbanization, typology of families, etc.) have recently occurred.

This study was designed as a cross-sectional survey of a nationally representative sample of adults $(>18$ years old) living in Spain. In summary, in 2015 a questionnaire of 50 items was specifically developed focused on sociodemographic aspects, food eating patterns, shopping habits, cooking habits and skills, and on nutrition knowledge and perception. In 2017, a follow up was run with these same items, but also questions about food decision and sustainability were added. The survey was administered by telephone by professionally trained surveyors via computer-assisted telephone interviewing (CATI). Participants resided in all territories of Spain, except for the Canary Islands and the Autonomous Cities of Ceuta and Melilla. A total of 2026 respondents (1223 women and 803 men) participated.

A summary of the main results are presented at the Congress: it is essential to highlight the importance of the role played by women. They are the main supporters concerned in preserving adequate dietary habits. Economic factors, more than health or food sustainability, are commonly considered by the population, although increasingly a healthy diet is considered similar to a sustainable one. Interestingly, a relatively high number of social and economical factors are shown to be associated with a normal weight achievement in the Spanish population. Undoubtedly social aspects are key elements to be considered in education and intervention nutrition policies.

This study was funded by Fundación MAPFRE (Madrid, Spain), a non-profit foundation committed to the development of people and social welfare. 


\section{Diabetes prevention through lifestyle changes and behavioural support: the PREVIEW Project.}

\section{Santiago Navas-Carretero}

Department of Nutrition, Food Sciences and Physiology; Center for Nutrition Research, University of Navarra, 31008 Pamplona, Spain.

CIBERObn, Fisiopatología de la Obesidad y Nutrición, Instituto de Salud Carlos III, Madrid, Spain. IdiSNA, Navarra Institute for Health Research, 31008 Pamplona, Spain.

Introduction: Type 2 diabetes prevalence is alarmingly increasing worldwide, mainly due to the obesity pandemia, as type 2 diabetes and obesity are closely related. In this context, different approaches regarding obesity management and type 2 diabetes prevention have been prioritised in research, and some of them, as the DPS (Diabetes Prevention Study) in Finland, the DPP (Diabetes Prevention Program) in the USA, or the study DiOGenes in Europe have shown positive results in reducing the incidence of type 2 diabetes mediated by lifestyle strategies.

Objective:

The main objective of the PREVIEW intervention was to find an effective mean of management of obesity and prevention of type-2 diabetesover the whole lifespan. Specifically, we aimed at testing if a high-protein, lowGI (glycemic index) diet, in combination with moderate or high intensity physical activitycould reduce the incidence of type- 2 diabetes inpre-diabetic subjects compared with the currently recommended diet(i.e. less protein and a higher GI).

Methods:

The study was designed as a multicentre intervention, in8 centres: HEL in Finland, UCPH in Denmark, UM in the Netherlands, UNOTT in the United Kingdom, UNAV in Spain, MU in Bulgaria, UNSYD in Australia and UOA in New Zealand. The subjects recruited would be allocated into one of the four groups: High-Protein and Moderate-Physical Activity, High-Protein \& High-Physical Activity, Moderate-Protein \& Moderate Physical Activity or Moderate-Protein \& High-Physical Activity. Behavioural changes were assessed with the PREMIT tool.

Results:

A total of 2,223 adults were randomised in the study, and $40 \%$ remained until CID7 (last Visit), but if we stick to those succeeding the $8 \%$ weight loss $(n=2,022)$, a $47 \%$ of those achieving weight loss arrived to the end of the study.Preliminary differential results among the four groups are modest, mainly due to the high effectiveness of the four groups, in which type 2 diabetes incidence was below $5 \%$, which demonstrates that the behavioural intervention was effective.

\section{Conclusion:}

Although data remain to be analysed, it can be said that adhering to a weight maintenance programme may be effective for reducing incidence of type 2 diabetes, independently of protein content and physical activity intensity, which may be effectively acquired through applying behaviour change techniques, as demonstrated with the PREMIT tool.

Keywords: PREVIEW project; Type 2 diabetes prevention; Diet and Physical Activity; Behaviour change. Conflict of interest: The authors declare they do not have a conflict of interest. The PREVIEW Project was funded by the Seventh FrameworkProgramme of the European Community forResearch, Technological Development andDemonstration Activities (2007 to 2013),KBBE.2012.2.2-03 Impact of lifestyle on wellbeingand diet-related disease.

17

The role of alcohol consumption on microbiota in healthy subjects. The ERAB Study.

\section{Ascensión Marcos Sánchez}

Immunonutrition Research Group. ICTAN. Consejo Superior de Investigaciones Cientificas (CSIC). Spain.

Introduction: Nowadays, there is a high controversy about the effect of alcohol consumption on health.The scientific literature on harmful effects of "binge drinking" on different organs is vast, although the study of moderate consumption of alcohol is acquiring a great interest even to evaluate possible benefits. On the other hand, microbiota has been classified as a new organ linked to homeostasis and metabolism, whose composition depends on several factors of lifestyle, such as diet. Objective and methods: Based on these concepts, we performed a study on 261 healthy adults to find out the role of the consumption of several alcoholic drinks on the gut microbiota.Alcohol consumption habits were analyzed by a specific questionnaire, and subjects were divided according to the following criteria: 1) Amount of alcohol (AoA): a) 
None-Low AoA (N=124), average consumption <5g alcohol/day; b) Moderate AoA: $\geq 5 \mathrm{~g} / \mathrm{d}$ and $\leq 20 \mathrm{~g} / \mathrm{d}$ (men) or $\leq 12$ g/d (women) (N=99); c) High AoA: > 20 g/d (men) or > 12 g/d (women) (N=38); and 2) Beer consumption: a) Abstemious ( $<1,5 \mathrm{~g}$ alcohol/día; $\mathrm{n}=44$ ); b) Beer consumers ( $>70 \%$ beer intake of the total alcohol consumption, wine intake $<2 \mathrm{~g} /$ dayand spirits $<6 \mathrm{~g} / \mathrm{d} ; \mathrm{n}=34$ ). Gut microbiota composition was analysed by 16sRNA sequencing (V3+V4, Illumina). Results: Main phyla and genus were not associated with different alcohol intake, but some differences were observed in minor groups ( $<\% 5$ abundance). Indeed, the high consumption group showed higher levels of Borrelia, Caldanaerobacter and Bulleidia compared to the moderate group ( $\mathrm{q}=0.011,0.040$ and 0.050 , respectively). In addition, beer intake showed positive associations with Bulleidia and Erysipelothrix, whereas wine was not associated to any bacterial group. Since beer seemed to be related to certain bacterial groups, we aimed to analyze differences between beer consumers and abstemious subjects. In this sense, the familyClostridiaceaeshowed higher levels in beer consumers compared to abstemious $(\mathrm{P}=0,029)$, as well as the genus Blautia and specificallyBlautia coccoides $(\mathrm{P}=0.027$ and $\mathrm{P}=0.044$, respectively). Conclusions:Although there were not associations between the alcohol consumption patterns established in this study and the main phyla and genera, the changes observed in minor bacterial groups ( $<5 \%$ abundance) may suggest that microbiota could be affected more by a high consumption of alcohol than by a moderate or regular consumption of alcohol. Further studies are necessary to find out the meaning and consequences of these results.

\section{Reversion of non-alcoholic fatty liver disease (NAFLD) by means of customized nutritional and lifestyle intervention: The FLIPAN study.}

\section{Josep A. Tur Marí}

1Research Group on Community Nutrition and Oxidative Stress, University of the Balearic Islands \& CIBEROBN, Palma de Mallorca, Spain.

Introduction: Non-alcoholic fatty disease (NAFLD) is the leading chronic liver disease, being characterized by lipid accumulation in the liver, which may progress to liver fibrosis, steatohepatitis (NASH), and cirrhosis. There are few treatment options, with exercise and diet featuring as the most widely advocated and practiced approach. However, there is insufficient data on the best exercise and diet approach, and there are few non-lifestyle therapies apart from bariatric surgery to induce weight loss and subsequent disease remission.

Objective: This application focuses on a personalized diet and exercise intervention with the expectation that this approach will be effective in decreasing body weight and fat, and reversing NAFLD.

Subjects \& Methods: The study is set up as multicenter RCT with 150 participants randomized to standard/control diet (AHA), Mediterranean diet plus high meal frequency, or Mediterranean diet plus exercise. The primary outcome is body weight and fat loss, and NAFLD status assessed by NMR/ultrasound/elastography. Secondary outcomes include biomarkers, liver enzymes, body composition, and quality of life measures. The intervention and data collection will be done at baseline, 6, 12 and 24 months.

Results: Preliminary results showed efficacy on reversal of body weight and fat, as well as NAFLD markers.

Conclusion: Benefits and impact of the study include new knowledge for diet/exercise recommendations and strategies for treating/preventing NAFLD and enhanced societal benefit due to reduced health burden and cost.

Acknowledgements:This study was fundedbyFundació La Marató TV3 (project201630.10), Health Department of Navarra Government (project 61/2015) and CIBEROBN(CB12/03/30038 and CB12/03/30002). 


\section{Genetic and epigenetic of childhood obesity}

\section{Concepción Aguilera García}

Department of Biochemistry and Molecular Biology . Institute of Nutrition and Food Technology.
University of Granada. Spain.

Obesity is a prevalent health problem affecting modern societies. It is particularly severe for children in developedcountries.Family and twin studies have suggested that obesity is $40 \%$ to $70 \%$ heritable, yet genome-wide association studies (GWAS) for BMI have identified only a small fraction of the implied genetic substrate for human obesity. There is an increasing evidence for the role of epigenetic factors in the development of obesity. Epigenetics is defined as heritable modifications in the expression of genes that can not be explained by changes in the ADN sequence. Epigeneticchanges are modulated by environmental exposure (including nutrition and physical activity), so that epigenetics is presented as a possible factor involved in the development of diseases such as obesity. Currently the study of obesity epigenome focuses mainly on the analysis of ADN methylation patterns, particularly due to the stability in the extracted ADN compared to other epigenetic marks, such as histone modifications. In addition, due to its biological plasticity, it is an attractive biomarker with great potential for clinical utility. One of the tools that is helping to know the epigenetic characteristics of obese individuals are studies of type EWAS (epigenome-wide association study), which are characterized by analyzing thousands or millions of CpGs in a certain number of individuals. The realization of EWAS in peripheral blood of children and its relationship with obesity is of great interest both for the study of the influence of maternal obesity and for the detection of epigenetic markers in childhood in order to make an early diagnosis of development of the disease and its metabolic complications. The validity of this type of studies is evident. Several studies have demonstrated the stability of epigenetic marks in the form of ADN methylations during the first years of life, as well as the influence of environmental factors during pregnancy. The objectives of this communication are: 1) to understand the molecular basis of the regulation of gene expression through epigenetic modifications. 2) to describe the main results of epigenome-wide association studies in peripheral blood associated with obesity in both adults and children.

Keywords: genetic, epigenetic, ADN methylation, obesity, adipose tissue, human

20

\section{Pregnancy and Fetal Programming}

\section{Elvira Larqué Daza}

University of Murcia. Spain.

An individual is born at conception and not at delivery. According to the Barker's hypothesis of the early onset of human metabolic diseases, early life experiences may induce permanent changes in structures and functions of certain organs and tissues, through a process of biological programming of the foetus. Several prenatatal and postnatal factors are associated with an increased risk of developing cardiovascular diseases and related co-morbidities such as obesity. Maternal lifestyle during pregnancy is considered an important relevant factor for childhood obesity preventative efforts. There are several models for the prediction of childhood overweight and obesity, but most of them have not been externally validated and the factors considered differ greatly among studies since the outcomes are predicted at different ages. Actions to be considered are promoting healthy nutrition and normal weight status at reproductive age and during pregnancy, monitoring infant growth carefully in order to detect excessive weight gain. During pregnancy, women should achieve proper gestational weight gain, and to avoid malnutrition, smoking, and free sugar intake higher than $10 \%$ energy. It is important also to consider the maternal nutritional habits during lactation, since maternal diet may influence both maternal milk nutrient composition and microbiota. 


\title{
Innovation activities: new beverages, reformulation and alternatives for a good choice for every moment and occasion
}

\author{
Rafael Urrialde de Andrés
}

Health and Nutrition Department. Coca-Cola Iberia

Since las 20 years, we have changed the way for working from food safety to nutrition knowledge. The risk analysis is a process consisting of three interconnected components: risk assessment, risk management andrisk communication. This is basic for food safety while ingredients, nutritional value and behavior are the most important elements for nutritional parameters. New beverages, new formulas with other current nutritional values for people need including reformulation, can be a good tool to manage the diet intake. We have and will have more choice with many alternatives to complete the food and beverage portfolio. The most important value for new reformulation is for reducing Sugar, fata, saturated fatty acids and salt values and the same time to maintain full taste. Reduction sweet taste is other goal for new dietetic paraments for helping to reduce specific nutrients. We have many kinds of consumers and the element to choose a food and beverage can be different person by person and region by region. There is reformulation pledge national food safety and nutrition authority with food and beverages industry for reducing $10 \%$ of several nutrients as added sugars, fat and salt in the Spanish diet. These goals are including tin the NAOS strategy for helping to energy and nutrient intake. Finally, the innovation activities are linked with information and communication claims now. The information and communication claims under the European and Spanish Regulations. They are: low energy, energy-reduced, energy-free, low-fat, fat free, low saturated fat, saturated fat-free, low sugars, sugars-free, with no added sugars, low sodium/salt, very low sodium/salt, sodium free or salt free, reduced (name of the nutrient), light/lite and they are other for saying bout benefits of nutrients and substance with physiology effects. It is needing to coordinate the innovation, reformulation and products activities with conditions of European and Spanish Regulations

References

Urrialde R. Salt, fat and sugar reduction in the food, for getting the goals for Spanish population. Nutr Hosp, 2018;35(№ extra 4):116-120. Doi: http://dx.doi.org/10.20960/nh.2136

Unión Europea. Reglamento (CE) n.. $1924 / 2006$ del Parlamento Europeo y del Consejo de20 de diciembre de 2006, relativo a las declaraciones nutricionales y depropiedades saludables en los alimentos. Diario Oficial de la Unión Europea, 2006;404:9-25.

\section{Contribution of artichokes to the nutritional well-being}

\author{
Perla Gómez Di Marco
}

Universidad Politécnica de Cartagena (IBV-UPCT-Murcia). Spain.

Introduction: Used as a food and a medicine from centuries, artichokes have an extensive history. Stems, leaves and heart inside play an important role in human nutrition, especially in the Mediterranean area. Recently, they have increasing significance due to its potential effects on human's health.

objectives: -To review the relationships between artichoke's bioactive compounds and their effects on healthiness. -To discuss about the technology available for the food industry to preserve artichoke's quality after harvest.

Methods: Scientific evidence was reviewed to highlight most relevant information related to the objectives. Studies carried on by our Research Institute for keeping artichoke's quality were also summarized.

Results: Artichokes are low in fat while rich in vitamins, minerals, and having some of the highest concentrations of antioxidants and fiber of any vegetable. The main phenolic compounds are the caffeic acid derivatives, including caffeoylquinic acid derivatives, as cynarin. Chlorogenic acid is the main monocaffeoylquinic acid, whereas luteolin-7-0-glucoside is the major flavonoid. All of them have shown in pharmacological test systems antioxidative, liver-protective, anti-microbial and lipid-lowering effects. Cynarin is known to stimulate the production of bile, which enables to digest fats and absorb vitamins. Flavonoids have also anti-inflammatory activity. The luteolin glucosides with caffeoylquinic acids played a crucial role in the inhibiting effect on the-novo synthesis of cholesterol and reduced lipoprotein low density (LDL) oxidation, being responsible of antiatherogenic action. Inulin, a fructan complex, also plays an important nutritional role in regulating sugars metabolism. There are other polyphenol-type antioxidants that could contribute to prevent and manage prostate cancer, breast cancer, and leukemia. In-vitro studies have shown that antioxidants rutin, quercetin, and gallic acid are able to induce apoptosis and reduce the proliferation of cancer cells. 
Although most artichoke recipes call for the vegetable to be steamed, stir-fried or braised, they can also be processed by less degradative methods.

Conclusions: several studies have proven that artichokes are a possible way to maintain and improve general health by influencing on many essential physiological functions. Technology available for preserving quality is safe and effective, even when it can be further improved.

Conflict of interest: Author declares no conflict of interest.

Key words: Cynara scolymus / disease prevention / antioxidants / health /

Juices: 100 \% Fruit, 'LiquidHealth to Drink'

$M^{a}$ Cruz Arcas Miñarro

AMC Innova. Spain.

AMC Innova is the Scientific-Technical unit of AMC Natural Drinks, in charge of all R\&D activities in the company.

Through itshealth, nutrition and sustainability policies, AMC Innova is committed to comply with the European nutritional guidelinesthroughout the whole R\&D process.

From our deep commitment of improvingconsumers quality of life, we are part of all European selfregulatory bodiesas well as the Fruit Juice MattersPlatform,dedicated tosupply relevant scientific content, endorsed by the most prestigious international universities; demonstrating the benefits of $100 \%$ fruit and vegetable juices and how they can help to improve population health. Worth noting that $100 \%$ juices are only squeezed fruit, with no added sugars, additives or preservatives, only containing the vitamins and minerals naturally present in fruits and vegetables and, therefore, full of nutritional benefits.

According to the above, the scientific community concludes that juice is not a sugary drink due to its complex matrix, that receives direct influence from the nutritional composition of the natural raw material from which it comes. Compounds involved in the benefits of juice are, among others: carotenoids, flavanones, phenols, vitamins, minerals, soluble and insoluble fibre.

Due to the synergistic effect of these compounds in a single glass of juice, it has been demonstrated that its intake is not related to a weight increase, and also that: it has a low glycaemic index; itspotassium content helps maintain blood pressure; regular juice consumers have lower levels of uric acid; there is no relationship between consumption and type II diabetesand it has favourable effects on cholesterol reduction.

24

Meat as a functional food. The AFUSAN group

\section{Francisco Sánchez-Muniz}

Departamento de Nutrición y Ciencia de os Alimentos. Facultad de Farmacia. Universidad Complutense de Madrid.

Meat/meat products are recognized human foods with cultural and nutritional importance. Their composition is highly dependent on several aspects (animal type, age, gender, part, size, feeding, sacrifice season, etc), thus, any generalization can be misleading. They are especially rich in protein (16-25\%); fat (11-30\%), and minerals (e.g. Fe, Zn) and vitamins (niacin, tiamin, retinol, B6 and B12); thus contributing to cover a large percentage of intakes and RDAs in meat consumers. Present nutritional guidelines recommend reducing meat/meat products consumption due to their saturated fat/cholesterol content. The search of extra-value meat with health implications is an important topic. Functional meats are meat that in addition to its intrinsic nutritional value are able to improve body functions and reduce degenerative disease risk. Animal genetic selection, feeding and postmortem actuation on reducing/ substituting "bad" components, and incorporating bioactive ingredients in meat can be involved. The AFUSAN group, Spanish acronym of "Functional foods, Health and Nutrition", is researching in obtaining healthier meat-products by incorporating several bioactive food/ingredients (walnut, seaweeds, glucomannans, spirulina, chia-oil, hydroxytyroxol, silicon and carob fruit extracts) to different meat types. Those meat-products have been tested in high-cardiovascular-disease-risk subject and in animal models (growing, aging, gene conditioned for obesity/metabolic syndrome) feeding them with high-energy/high-saturated and cholesterol diets. Results demonstrated that meat is an adequate matrix for incorporating functional ingredients that would permit, without reducing its consumption, to partially block the potential negative effects related to saturated fat/cholesterol content. In our most recent research we have investigated on the benefits of 
including silicon-enriched-meat in the frame of those high energy/high saturated and cholesterol diets on some biomarkers of non-alcoholic-fatty-liver disease, considered a clear manifestation of the metabolic syndrome. Silicon-enriched-meat reduces lipotoxicity and their oxidative and inflammatory linked mechanisms suggesting the utility of incorporating silicon to obtain functional and healthier meat-products.

Parcially supprted by Santander-UCM PR75/18-21603 project. 


\title{
The effect of the ToyBox-intervention on parental perceptions, attitudes and knowledge on European preschool children's screen time: The ToyBox-study
}

\author{
M.L. Miguel-Berges ${ }^{a}$, A.M. Santaliestra-Pasias ${ }^{a}$, T. Mouratidou ${ }^{a}$, O. Androutsos ${ }^{b}$, M. Craemer ${ }^{c}$, S. Galcheva ${ }^{d}$, \\ Koletzko B', L.A. Moreno ${ }^{a}$ \\ ${ }^{a}$ Growth, Exercise, Nutrition and Development (GENUD) Research Group, University of Zaragoza, Zaragoza, \\ Spain. \\ ${ }^{\mathrm{b}}$ Department of Nutrition and Dietetics, School of Health Science and Education, Harokopio University, Athens, \\ Greece. \\ 'Department of Movement and Sports Sciences, Ghent University, Belgium. \\ dMedical University Varna, Varna, Bulgary. \\ 'Dr von Hauner Children's Hospital, University of Munich Medical Centre, Munich, Germany.
}

Background: Preschool children spend high proportions of hours being sedentary. Screen time (e.g. television/DVD viewing and computer/video games use) is negatively associated with several health outcomes, but interventions aiming to reduce preschool children's sedentary time are scarce. Parents play a critical role in developing and shaping their children's sedentary behaviour, particularly in the early years of life. Objectives: To assess parental perceptions, attitudes and knowledge of their preschool children's sedentary behaviour and the association with children's time spent on TV/video/DVD/video games and total screen time. Methods: Data was obtained from 4836 children aged 3.5 to 5.5 years, participating in the multi-centre ToyBox-study during the baseline and follow-up measurements. Data on children's sedentary behaviour was collected via the standardized proxy-administered parental questionnaire. Multilevel logistic regression was used to examine the associations between parental perceptions, attitudes and knowledge and time spent on TV/video/DVD/video games and total screen time of their children. Results: Regarding total screen time, $66.6 \%$ of the sample exceeded the recommendations at $\mathrm{T} 0$, and $71.8 \%$ at $\mathrm{T} 1$ in the control group, whereas in the intervention group the proportion varied from $69.7 \%$ at $\mathrm{T} 0$ to $72.5 \%$ at $\mathrm{T} 1$. The odds of exceeding the total screen recommendations in preschool children were significantly higher when parents did not set rules on screen-viewing/using for their children, in the intervention and control groups $(\mathrm{p}<0.05)$. Also, the odds of exceeding the TV/video/DVDs viewing's recommendations were significantly higher when parents thought (at T0 and T1) that the recommendations were to spend more than 3 hours per day. Conclusion: Preschool children whose parents have rules limiting their sedentary time were less likely to spend a high amount of time at TV/video/DVD/video games, whereas children with permissive parents were more likely to have high levels of TV/video/DVD/video games viewing. Improving parenting practices may be a promising approach to decrease screen time of preschool children. Conflict of interests: None.

\section{Breakfast characteristics and its association with daily macronutrient intake. A systematic review and meta-analysis}

N. Giménez-Legarre ${ }^{a, b}$, P. Flores-Berrantes, ${ }^{a, b}$, M.L. Miguel-Berges ${ }^{a, b}$, A.M. Santaliestra-Pasías ${ }^{a, b, c}$, L.A. Moreno ${ }^{a, b, c}$

${ }^{a}$ GENUD (Growth, Exercise, Nutrition and Development) Research Group, Facultad de Ciencias de la Salud, Universidad de Zaragoza, Zaragoza (España).

Instituto Agroalimentario de Aragón (IA2), Instituto de Investigación Sanitaria Aragón (IIS Aragón) España.

'Centro de Investigación Biomédica en Red de Fisiopatología de la Obesidad y Nutrición (CIBERObn). España.

Background: Breakfast (BF) consumption has been associated with favourable health outcomes, which includes a high proportion of nutrient intake adequacy or a low risk for chronic diseases. Objectives: To investigate the associations between BF consumption and its relationship with daily energy and macronutrients intake. Methods: An exhaustive search was carried out in three datasets in March 2019. 
Two independent reviewers performed the data extraction and assessed quality and risk of bias following the PRISMA methodology and using the Cochrane risk of bias tool. Meta-analysis was performed comparing results based on type of BF: skip BF, Ready To Eat Cereal (RTEC) BF and other types of BF. DerSimonian and Laird estimators using random effects models were applied for continuous data. Effect sizes were calculated for each outcome. Results: Out of 3105 articles, we identified 31 full-text articles for inclusion and 7 were considered for meta-analysis. The mast common topic was RTEC consumption. Children who usually skip BF had significantly lower intake of energy (SMD,-5.414; 95\%CI: -8.126, -2.702), carbohydrates (SMD,-8.214; 95\%CI: -11.377, -5.052), fibre (SMD,-8.436; 95\%CI: -12.638, -4.234) and protein (SMD,-6.057; 95\%CI: $8.358,-3.756$ ) than children who usually have BF. However BF skippers had a higher fat intake (SMD,4.596; 95\%CI: $2.042,7.150)$. On the other hand, children who usually eat RTEC-BF had significantly lower fat intake (SMD,11.101; 95\%CI: 7.159, 15.044), and higher intake of energy (SMD,-7.005; 95\%CI: -11.512, -2.498), carbohydrates (SMD,-9.287; 95\%CI: -13.448, -5.125), fibre (SMD,-6.672; 95\%CI: -11.023, -2.321) and protein (SMD,-3.032; 95\%CI: $-4.613,-1.452$ ) than children who usually skip BF. Conclusion: BF consumption is associated with better energy and macronutrient intake. RTEC have a direct association with energy, carbohydrates and protein intake. Conflict of interests: Authors declare no conflicts of interest.

\title{
Prevotella enterotype is associated with higher body mass index, HOMA level, and fiber intake
}

\author{
A. Cuevas-Sierra ${ }^{a}$, J.I. Riezu-Boj ${ }^{a}$, E. Guruceaga ${ }^{b}$, J.A. Martínez ${ }^{a}$, F.I. Milagro ${ }^{a}$
}

${ }^{a}$ Departamento de Ciencias de la Alimentación y Fisiología. Centro de Investigación en Nutrición. Universidad de Navarra. Pamplona, España.

${ }^{b}$ Departamento de Bioinformática. Centro de Investigación Médica Aplicada. Universidad de Navarra. Pamplona, España.

Background: Scientific research has evidenced that categorization of individuals according to microbial enterotypes could be a potential tool in personalized nutrition and obesity management. Objectives: To analyze the enterotypes in an adult population, and their influence on body mass index (BMI) and insulin resistance (HOMA-IR). Methods: Baseline fecal and blood samples of 64 normal weight $\left(\mathrm{BMI}<24.9 \mathrm{~kg} / \mathrm{m}^{2}\right)$, 110 overweight (25-30 kg/m²) and 186 obese ( $\left.>30 \mathrm{~kg} / \mathrm{m}^{2}\right)$ Spanish subjects were collected. DNA from fecal samples was sequenced with the Illumina $16 \mathrm{~S}$ protocol, based on the amplification of the V3-V4 variable regions of the 16S rRNA gene. The microbial classification was performed by targeted clustering using methods described by Arumugam et al. (2011). Fiber intake was estimated with a food frequency questionnaire (Shatenstein et al., 2005). ANOVA and ANCOVA tests adjusted by sex and total energy intake were performed. Results: Three enterotypes were found in this population: Bacteroides, Prevotella and Oscillibacter. ANOVA analysis showed significant differences in mean BMI, HOMA-IR and fiber intake between the three enterotypes. Post hoc analyses showed that Prevotella enterotype had significantly higher BMI, HOMA-IR and fiber intake than Bacteroides. ANCOVA test showed that subjects with the Prevotella enterotype had higher values of BMI $(p<0.001)$, HOMA-IR $(p=0.043)$ and fiber intake $(p<0.001)$ than Bacteroides+Oscillibacter. Conclusion: Prevotella enterotype could be relevant in the development of obesity and insulin resistance, and is directly related to fiber intake. Conflict of interests: Authors declare no conflicts of interest. Acknowledgements: This research was funded by CIBERobn and Government of Navarra (Microbiota project, PIO38, and Nutribiota project). 


\title{
Comparative analysis of the anthropometric profile of female elite basketball and volleyball players
}

\author{
A. Miguel-Ortega ${ }^{a}$, J.M. Calleja-González ${ }^{b}$, J. Mielgo-Ayuso ${ }^{c}$ \\ ${ }^{\mathrm{a}}$ Facultad de Humanidades y Ciencias Sociales. Universidad Isabel I. Burgos, España. \\ ${ }^{b}$ Facultad de Educación y Deporte. Universidad del País Vasco. Vitoria, España. \\ 'Escuela Universitaria de Fisioterapia. Universidad de Valladolid. Soria, España.
}

Background: Body composition discharges a crucial role on the physical performance of basketball and volleyballers. Anthropometric profiles are particularly important in both disciplines because absolute size contributes significantly to the total variance associated with athletic success. Objectives: Analyze the anthropometric profile, body composition and physical performance of female elite level volleyball and basketball players over a season. Methods: Age, height, body mass and anthropometry data were collected from 11 volleyball players and 12 from basketballers (both female elite level groups) (age: 25,111 \pm 4,656 yr). The anthropometric measures collected were: fat mass ( 8 folds), musculoskeletal mass (11 perimeters) and 3 bone diameters. The parameters of physical performance examined were: jump test (SJ, CMJ, ABK, DJ), speed $20 \mathrm{~m}$, agility (Illinois test), medical ball throw (3 kg), RSA test with change of direction and test of intermittent endurance. Results: It has been observed that although the average intergroup height only differs by $1,742 \mathrm{~cm}$, the intergroup body mass average differs by more than eight kilograms. In turn, the basketball group presented a lower level of fat mass $(19,916 \pm 4,440)$ and residual mass $(69,761 \pm 13,624)$ as well as higher values of muscle mass $(10,284 \pm 13,810)$ and bone mass $(0,038 \pm 0,061)$ vs. volleyball group $(20,145 \pm 7,930,72,797 \pm 7,696,7,037 \pm 0.909$ and $0.021 \pm 0.003)$. The analysis of the data indicates that the anthropometric and compositional characteristics of both groups respond with the recommendations associated with the somatic characteristics of their respective sports. Conclusion: These results show the need for education in the field of nutrition as well as the need for further research on dietary habits among athletes. It is recommended to increase muscle mass and slightly reduce body fat. Conflict of interests: The authors declare no conflict of interests.

\section{Preventive effects of folic acid supplementation on ototoxicity in mice}

C. Moráis-Moreno ${ }^{a}$, M.P. Garzón-Riveros ${ }^{b, c}$, S. Murillo-Cuesta, ${ }^{d, e}$, M.A. Pajares ${ }^{f}$, J. Pérez-Miguelsanz ${ }^{g}$, I. VarelaNieto $^{d, e}$, G. Varela-Moreiras ${ }^{a}$, T. Partearroyo ${ }^{a}$

aDepartamento de Ciencias Farmacéuticas y de la Salud, Facultad de Farmacia, Universidad San Pablo-CEU, CEU Universities. Madrid, Spain.

${ }^{b}$ Departamento de Anatomía. Facultad de Medicina. Universidad Complutense de Madrid. Madrid, Spain. "Instituto de Investigaciones Biomédicas "Alberto-Sols" (CSIC-UAM)

${ }^{d}$ Centro de Investigación Biomédica en Red (CIBERER)

e Instituto de Investigación Sanitaria del Hospital Universitario La Paz (IdiPAZ). Madrid, Spain.

fDepartamento de Biología Estructural y Química, Centro de Investigaciones Biológicas (CSIC). Madrid, Spain. ${ }^{9}$ Departamento de Anatomía. Facultad de Medicina. Universidad Complutense de Madrid. Madrid, Spain.

Background: Background: According to the World Health Organization, the incidence of auditory disorders, especially noise-induced hearing loss (HL), is increasing dramatically. In addition to genetic and environmental ethiologic factors such as noise and ototoxic drugs, nutritional imbalance is an emerging contributor. Epidemiological and experimental studies revealed a correlation between HL and increased plasma homocysteine (Hcy) secondary to methionine cycle impairment. Betaine homocysteine Smethyltransferases (BHMTs) and methionine synthase are responsible for Hcy remethylation, hence their decreased levels and/or malfunction lead to hyperhomocysteinemia (HHcy). Objectives: Analysis of the impact of an ototoxic drug (paracetamol; APAP) in cochlear morphology and function of control and Bhmt-/mice, as well as potential preventive effects of folic acid (FA) supplementation. Methods: Two-month-old Bhmt-/- mice ( $\mathrm{n}=47)$, with greater dependence on folate metabolism for Hcy remethylation, and Bhmt+/+ mice $(n=42)$ were fed control or FA supplemented diets for 30 days. The last day APAP (250 mg/kg) or placebo were injected intraperitoneally. Hearing was evaluated by recording auditory brainstem responses 
at the beginning of the experiment and after treatments. Picrosirius red staining was used for evaluation of the cochlear lateral wall cytoarchitecture. Plasma and hepatic metabolite levels were determined by HPLC or on Spinlab $100 \circledR$ autoanalyzer. Results: Loss of Bhmt expression exerted no evident impact in mice hearing acuity. Single APAP administration did not induce ABR threshold shifts. However, following ototoxic treatment, the stria vascularis and spiral ligament surface areas changed 5-17\% between Bhmt-/- mice under different dietary treatments; cochlear structures of Bhmt-/- mice receiving APAP plus FA supplementation resemble those of the control group. Enhanced susceptibility to APAP ototoxic damage was associated with HHcy. Conclusion: BHMT plays a central role in cochlear methionine metabolism. Nutritional interventions with FA could be of protective value for human hearing by modulating Hcy levels. Conflict of interests: The authors declare no conflict of interest.

6

\title{
Influence of water intake and balance on body composition
}

\author{
A.I. Laja-García ${ }^{a}$, C. Moráis-Moreno ${ }^{a}$, M.L. Samaniego-Vaesken ${ }^{a}$, T. Partearroyo ${ }^{a}$, G. Varela-Moreiras ${ }^{a}$
}

aDepartamento de Ciencias Farmacéuticas y de la Salud, Facultad de Farmacia, Universidad San Pablo-CEU. Madrid, Spain.

Background: At present, obesity and overweight are major public health concerns. It is known that their classical determinants (diet and physical activity), do not sufficiently explain its high prevalence. In this regard, in the last years several investigations have suggested that water intake and water balance could have important implications for weight management and body composition. Objectives: The aim of this study was to examine the effect of water intake and water balance on body composition in healthy young adults from Spain. Methods: The study involved 358 healthy subjects (121 males; 237 females) aged between 18-39 years. Water intake was assessed through "the hydration status questionnaire". Anthropometric measurements (height, weight and waist circumference) were acquired according to the recommendations of the International Standards for Anthropometric Assessment. The estimates of total body water (TBW), fat mass (FM) and fat free mass (FFM) were estimated by bioelectrical bioimpedance analysis. Differences in anthropometrics and body composition variables were assessed through the ANOVA test and considered significant at $\mathrm{p}<0.05$. Results: Total water intake was correlated with body water content in both, females $(r=0.180, p=0.006)$ and males $(r=0.266, p=0.003)$. We found an inverse association between the consumption of water $(\mathrm{ml}) / \mathrm{kg}$ per weight with body weight, body fat mass and waist circumference in females and males. In addition, we observed significant differences in body water content in females according to water balance. Conclusion: Higher consumption of water seems to be related with a better body composition. Therefore, adequate water intake could contribute to prevent overweight and obesity in young adults. Conflict of interests: The authors declare no conflict of interest.

\section{7}

\section{PREDIMED-Plus trial: One-year changes in the quality of dietary carbohydrate intake and concurrent changes in cardiovascular risk factors}

\author{
C.I. Fernández-Lázaro ${ }^{a}$, E. Toledo ${ }^{a}$, J. Salas-Salvadó ${ }^{b}$, D. Corella ${ }^{c}$, M. Fito ${ }^{d}$, J.A. Martínez ${ }^{e}$, P. Buil-Cosiales ${ }^{d}$
}

aDepartment of Preventive Medicine and Public Health, IDISNA, University of Navarra, Pamplona, Spain. ${ }^{b}$ Department of Biochemistry and Biotechnology, Universitat Rovira i Virgili, Nutrition Unit, Reus, Spain. 'Department of Preventive Medicine, University of Valencia, Valencia, Spain.

${ }^{\mathrm{d} C I B E R}$ Physiopathology of Obesity and Nutrition (CIBERObn), Carlos III Institute of Health, Madrid, Spain.

eDepartment of Nutrition, Food Sciences, and Physiology, Center for Nutrition Research, University of Navarra, Pamplona, Spain.

Background: cardiovascular disease (CVD) is the worldwide leading cause of morbi-mortality. Quality of dietary carbohydrate (CHO) intake rather than quantity may play an important role on reducing certain CVD risk factors such as overweight/obesity, hypertension, hyperglycemia, and hypercholesterolemia.. Objectives: To examine the effect of 6 and 12-month changes in dietary quality of CHO on CVD risk factors in the participants of the PREDIMED-Plus, a multicenter, randomized, lifestyle, primary prevention trial of 
CVD. Methods: A total of 5,373 overweight/obesity Spanish adults (aged 55-75 years) with metabolic syndrome at baseline were allocated to a weight-loss lifestyle intervention based on an energy-restricted Mediterranean diet, physical activity promotion, and behavioural support (Intervention Group) or an energy unrestricted Mediterranean diet (Control Group). Four criteria (fiber intake, glycemic index, wholegrain/total-grain carbohydrate ratio, and solid/total carbohydrate ratio) were used to assess the dietary CHO quality intake. The relationship between changes in the carbohydrate quality index (CQI) and concurrent changes in risk factors was assessed. Results: The majority of participants improved their CQI by increasing their consumption of fruits, vegetables, and legumes, and decreasing their consumption of cereals, white bread, and soda drinks. After 6 months, body weight, waist circumference, systolic BP, diastolic BP, fasting blood glucose, HbA1c, and triglyceride levels monotonically decreased across successive quintiles of improvement in CQI. At 12-month follow-up CVD risk factor improvements were additionally observed for HDL-CH and for the ratio total cholesterol:HDL-cholesterol. The most important favorable risk factor changes (expressed in units of SD) between the upper and lower quintiles of CQI were observed for waist circumference $(-0.29 ; 95 \% \mathrm{CI}-0.34$ to -0.25$)$, weight $(-0.21 ;-0.24$ to -0.18$)$, and HbA1c $(-0.20 ;-0.26$ to -0.14$)$. Conclusion: Improvements in CQI were strongly associated with favorable CVD risk factor changes in the PREDIMED-Plus randomized intervention trial. Conflict of interests: None.

\title{
Long-term effects of a novel adiponectin agonist treatment of gestational diabetes in rats
}

\author{
A. Gázquez-García ${ }^{a}$, F. Rodríguez-Mulero ${ }^{a}$, L.E. Martínez-Gascón ${ }^{b}$, M.D. Albadalejo Otón ${ }^{b}$, E. Larqué Daza $^{a}$
}

${ }^{a}$ Department of Physiology, Biomedical Research Institute of Murcia (IMIB-Arrixaca-UMU), University Clinical Hospital "Virgen de la Arrixaca", University of Murcia, Murcia, Spain.

${ }^{b}$ Department of Clinical Analysis, Biomedical Research Institute of Murcia (IMIB-Arrixaca-UMU), Santa Lucia General University Hospital, Cartagena, Murcia, Spain.

Background: Gestational diabetes mellitus (GDM) reduces docosahexaenoic acid (DHA) in fetal plasma which could affect their development. Adiponectin agonists have shown anti-diabetic properties by enhancing insulin sensitivity. Objectives: To evaluate the effects of adiponectin agonist administration on glycaemia and fatty acids profile in pregnant diabetic rats and their long-term consequences in offspring. Methods: Pregnant Sprague Dawley rats were randomized in three groups: diabetic rats (GDM, n=11), diabetic rats treated with adiponectin agonist (GDM+ADI, $\mathrm{n}=12)$ and controls without GDM $(\mathrm{n}=13)$. Diabetes was induced with streptozocin $\left(50 \mathrm{mg} / \mathrm{kg}\right.$ ) and GDM+ADI received $50 \mathrm{mg} / \mathrm{kg}$ per day of AdipoRon ${ }^{\circledR}$ from day 14. Maternal plasma and fetus were collected at delivery. In some offspring at adult state (12wk), plasma and brain were also collected (GDM n=20, GDM+ADI $n=14$, Control $n=16$ ) for glycaemia and lipid analyses. Results are expressed as mean \pm SEM. Results: Adiponectin agonist GDM treatment tended to reduce fasting glucose level in pregnant diabetic rats (GDM: 168 $\pm 70 \mathrm{a} \mathrm{mg/dL,} \mathrm{GDM+ADI:} 139 \pm 40 \mathrm{ab} \mathrm{mg} / \mathrm{dL}$, Control: $91 \pm 64 \mathrm{~b}$ $\mathrm{mg} / \mathrm{dL}, \mathrm{P}=0.039)$. Fetal weight was significantly reduced by GDM $(\mathrm{P}=0.002)$, while total fat content tended to be increased in these groups (GDM: $6.83 \pm 0.33 \mathrm{mg} / \mathrm{g}$, GDM+ADI: $6.43 \pm 0.24 \mathrm{mg} / \mathrm{g}$, Control: $6.02 \pm 0.21 \mathrm{mg} / \mathrm{g}$, $\mathrm{P}=0.102)$. In offspring at 12 weeks of age, $90 \mathrm{~min}$ after an oral overload of glucose, maternal adiponectin agonist treatment also reduced plasma glucose levels (GDM: 102 $\pm 5 \mathrm{a} \mathrm{mg} / \mathrm{dL}, \mathrm{GDM}+\mathrm{ADI}: 93 \pm 14 \mathrm{ab} \mathrm{mg} / \mathrm{dL}$, Control: $81 \pm 4 \mathrm{~b} \mathrm{mg} / \mathrm{dL}, \mathrm{P}=0.010$ ). DHA content in plasma was significantly reduced in both GDM and GDM+ADI groups compared to controls (GDM: 3.16 $\pm 0.20 \mathrm{a} \%$; GDM+ADI: $3.32 \pm 0.18 \mathrm{ab} \%$; Control: $4.22 \pm 0.37 \mathrm{~b} \%, \mathrm{P}=0.013$ ), while no differences were observed in FA profile of brain, which seems to be strongly regulated. Conclusion: Adiponectin agonist administration in pregnant rats improves glycaemia in mothers and offspring while this treatment does not significantly improve lipid profile in the offspring of diabetic rats at long-term. Conflict of interests: All authors declare no conflicts of interest.

\section{Hydroxytyrosol supplementation impairs skeletal muscle glucose transport in trained rats: an analysis of the GLUT4/AKT axis}

\author{
J.R. Huertas ${ }^{a}$, S. Al-Farazi ${ }^{b}$, A. Rueda-Robles ${ }^{b}$, J. Aragón ${ }^{b}$, J. Plaza-Díaz ${ }^{c}$, R.A. Casuso ${ }^{b}$
}


anstituto de Nutrición y Tecnología de los Alimentos, Centro de Investigación Biomédica, Universidad de Granada, Granada, Spain.

"Institute of Nutrition and Food Technology "José Mataix", Biomedical Research Center, Department of Physiology, University of Granada, Granada, Spain.

'Department of Biochemistry and Molecular Biology II, School of Pharmacy, Campus de Cartuja s/n, University of Granada, Granada, Spain.

Background: Exercise improves skeletal muscle glucose uptake by increasing the expression of the glucose transporter 4 (GLUT4). However, a high antioxidant intake could hamper this process. It has been shown that hydroxytyrosol (HT), a powerful antioxidant found in olive oil; improves insulin sensitivity in vitro. Nevertheless, whether HT supplementation could prevent the exercise-induced adaptation on the GLUT4/AKT axis remains unknown. Objectives: Our purpose was to describe the effects of a medium and a high HT dose for 10 weeks on the GLUT4-AKT axis in exercised rats. Methods: 40 male Wistar rats were distributed in 4 groups for 10 weeks: Sedentary (Sed), Exercised (EXE), EXE with $20 \mathrm{mg} / \mathrm{kg} /$ day of HT (EXE20), EXE with $300 \mathrm{mg} / \mathrm{kg} /$ day of HT (EXE-300). At the end of the study, the animals were overnight fasted anesthetized and sacrificed. Soleus muscle was excised and frozen at $-80^{\circ} \mathrm{C}$. GLUT4 RNA levels were quantified by real time PCR, in addition GLUT4 total AKT and AKT phosphorylation at Tr308 (AKTp) was assessed by Western Blot. Results: The One way ANOVA showed the exercise increased the expression of GLUT4 as well as the AKTp/AKT ratio $(p<0.05)$. This effect was not observed in none of the supplemented groups. Nevertheless, the EXE-300 group showed a significant rise of the GLUT4 mRNA levels $(p<0.05$ ) while neither EXE nor EXE-20 showed an increment of the GLUT4 mRNA levels. Conclusion: Our results show that mild to high doses of HT supplementation blunts the expression of GLUT4 as well as the degree of AKT activation induced by exercise. The fact that the EXE300 group show an increased transcription of GLUT4 which is not translated into effective protein expression needs to be further explored. Conflict of interests: The authors declare no conflicts of interest. Acknowledgement: The experiments were approved by the ethics committee of the University of Granada (Granada, Spain; n: 28/06/2016/116).

10

\title{
Body composition based on bioelectrical impedance analysis and therapeutic exercise in fibromyalgia syndrome
}

\author{
L. Ceballos Laita ${ }^{a}$, T. Mingo-Gómez ${ }^{a}$, F. Navas-Cámara ${ }^{a}$, Z. Verde-Rello ${ }^{a}$, A. Fernández-Araque ${ }^{a}$, E. Estébanez-de- \\ Miguel $^{b}$, E. Bueno-Gracia ${ }^{b}$, S. Caudevilla-Polo ${ }^{b}$
}

${ }^{a}$ Facultad de Fisioterapia, Campus Universitario Duques de Soria, Universidad de Valladolid, Soria, España. ${ }^{\mathrm{b}}$ Facultad de Ciencias de la Salud, Universidad de Zaragoza, Zaragoza, España.

Background: Fibromyalgia syndrome is a chronic condition characterized by widespread musculoskeletal pain, fatigue and sleep disturbances. Overweight and obesity in women with fibromyalgia diagnosis may increase the pain and fatigue, as well as the risk for a variety of illnesses such as diabetes, hypertension and cardiovascular disease. Exercise therapy has shown positive effects on pain and physical function, but the effects on fibromyalgia patients is still unclear. Objectives: The aim of this study was to determinate the effects of 30 sessions of therapeutic exercise in body composition, pain, fatigue and sleep disturbances in a case series of women with fibromyalgia syndrome. Methods: Baseline data was collected on 16 women diagnosed with fibromyalgia according to the American College of Rheumatology. 3 sessions a week of therapeutic exercise based on American College of Sports Medicine for 10 weeks were performed between October to December. Body composition (\% muscle mass, \% fat mass and \% visceral fat) was recorded by bioelectrical impedance analysis (TANITA SC240). Pain intensity, fatigue and sleep disturbances were measured by visual analog scale. Results: 14 women completed the study, 2 women dropped out for personal decisions. The results of the study showed a statistical significative increase of the muscle mass $(\mathrm{p}=$ $0.04)$, a decrease of fat mass $(p=0.04)$ and an improvement of sleep disturbances $(p=0.04)$. Fatigue showed a tendency to improve after therapeutic exercise program $(p=0.05)$. No statistical differences were shown in pain intensity and visceral fat ( $p>0.05$ ). Conclusion: In conclusion, therapeutic exercise based on American College of Sports Medicine for 10 weeks seems to decrease fat mass, to increase muscular mass, and to improve fatigue and sleep disturbances in women diagnosed with fibromyalgia syndrome. Conflict of interests: Authors declare no conflicts of interest. 
Maresin 1 improves glucose homeostasis and insulin signaling in human adipocytes and in lean and obese mice

\author{
L. Martínez-Hernández ${ }^{a}$, P. González-Muniesa ${ }^{b, d c}$, N. Sáinz ${ }^{e}$, X. Escotée ${ }^{e}$ J.A. Martínez ${ }^{b, c, d}$, M.J. Moreno-Aliaga ${ }^{b, c, d}$ \\ aUniversidad de Navarra, Departamento de Ciencias de la Alimentación y Fisiología. Centro de Investigación en \\ Nutrición, Pamplona, España. \\ bUniversidad de Navarra, Departamento de Ciencias de la Alimentación y Fisiología. Centro de Investigación en \\ Nutrición, Pamplona, España. \\ 'CIBERObn Physiopathology of Obesity and Nutrition, ISCIII, Madrid, Spain. \\ 'IDISNA, Navarra's Health Research Institute, Pamplona, Spain. \\ eUniversidad de Navarra, Centro de Investigación en Nutrición, Pamplona, España.
}

Background: MaR1 is a DHA-derived lipid mediator with anti-inflammatory and proresolving properties that has been revealed to exert insulin sensitizing actions in murine models of obesity. Objectives: The first aim of the present study was to characterize the ability of MaR1 to prevent the alterations induced by the proinflammatory cytokine TNF- $\alpha$ on insulin actions on glucose uptake and Akt phosphorylation in cultured human adipocytes from overweight/obese subjects. In addition, we investigated the effects of both acute and chronic administration of MaR1 on Akt activation in the absence or presence of insulin in white adipose tissue (WAT) and skeletal muscle of lean and diet-induced obese (DIO) mice. Results: MaR1 (0.1 nM) prevented the inhibitory effect of TNF- $\alpha$ on insulin-stimulated 2-DG uptake and Akt phosphorylation in human adipocytes. Moreover, acute treatment with MaR1 (50 $\mu \mathrm{g} / \mathrm{kg}, 3 \mathrm{~h}$, i.p.) induced Akt activation in WAT and skeletal muscle of lean mice. However, MaR1 did not further increase the stimulatory effect of insulin on Akt activation. Interestingly, intragastric chronic treatment with MaR1 (50 $\mu \mathrm{g} / \mathrm{kg}, 10$ days) reduced the hyperglycemia induced by the high fat diet (HFD) and improved systemic insulin sensitivity. In parallel, MaR1 partially restored the impaired insulin response in skeletal muscle of DIO mice. Additionally, MaR1 reversed HFD-induced lower Akt activation in WAT in non-insulin-stimulated DIO mice, but did not restore the defective Akt activation in response to acute insulin observed in DIO mice. Conclusion: In summary, our results suggest that MaR1 attenuates the impaired insulin signaling and glucose uptake induced by proinflammatory cytokines. Moreover, the current data also support that MaR1 treatment could be useful to reduce the hyperglycemia and the insulin resistance associated to obesity, in part by improving Akt signaling. Conflict of interests: The authors declare they do not have any conflict of interest.

12

\title{
Endocrinometabolic disturbances of mice lacking SSAO/VAP-1 by Aoc3 gene deletion are mostly reproduced in mice expressing a vascular adhesion protein-1 (VAP-1) devoid of amine oxidase activity (SSAO)
}

\author{
V. Jargaud ${ }^{a, b}$, F. Tercée ${ }^{a, b}$, X. Collet ${ }^{a, b}$, P. Mauriège ${ }^{c}$, A. Bouloumié $^{a, b}$, S. Jalkanen ${ }^{d}$, C. Stolen $^{d}$, M. Salmi $^{d}$ \\ anstitute National de la Santé et de la Recherche Médicale, INSERM U1048, I2MC, Toulouse, France. \\ buniv. Paul Sabatier, Toulouse, France. \\ 'Dept. of Kinesiology, Fac. of Medicine and PEPS, Laval Univ., Québec, Canada. \\ ${ }^{\mathrm{d}}$ MediCity and Biotie Therapies, Turku, Finland.
}

Background: Vascular Adhesion Protein-1 (VAP-1) encoded by Aoc3 gene exhibits Semicarbazide-Sensitive Amine Oxidase activity (SSAO). VAP-1/SSAO is highly expressed in endothelial, smooth muscle and fat cells, while also found in blood. Its multifaceted role remains unclear, since it facilitates leucocyte adhesion, oxidizes endogenous or dietary amines, and generates hydrogen peroxide and aldehydes. Its involvement in immunity is now well described, leading to the development of VAP-1/SSAO inhibitors and antibodies as novel anti-inflammatory agents. However, the exploration of VAP-1/SSAO functions connected with metabolism regulation is still incomplete. Objectives: In an attempt to decipher the respective role of adhesion molecule and that of amine oxidase generating highly reactive products, we compared the phenotype of mice with total Aoc3 gene knock-out (AOC3KO) and of mice expressing a mutated VAP-1 lacking SSAO activity (knock-in line called AOC3KI). Methods: In an attempt to decipher the respective role of adhesion molecule and that of amine oxidase generating highly reactive products, we compared the phenotype of mice with total Aoc3 gene knock-out (AOC3KO) and of mice expressing a mutated VAP-1 
lacking SSAO activity (knock-in line called AOC3KI). Results: In both lines, no tissue SSAO activity was found, while AOC3 mRNA was absent in adipose tissues (AT) of AOC3KO only. Although food consumption was unchanged, both genders of AOC3KO and AOC3KI were heavier and fatter than respective controls. Other common features were found in AT: loss of benzylamine insulin-like effect in adipocytes, unchanged insulin sensitivity and adiponectin expression, inflammatory marker down-regulation (CD45, IL 6). Glucose tolerance and liver mass remained unchanged, while circulating lipid profile was distinctly altered, with increased cholesterol in AOC3KO only. Conclusion: These results suggest that the lack of primary oxidase activity of mutated VAP-1/SSAO is sufficient to reproduce the changes in metabolic homeostasis observed in AOC3KO mice, save those related with cholesterol handling. Conflict of interests: Authors declare no conflicts of interest with this work.

13

\title{
Metabolic alterations in IGF1R-deficient mice: effects of ageing and obesity
}

\author{
P. Pérez-Matute ${ }^{a}$ I. López ${ }^{b}$, M. Íñiguez ${ }^{c}$, E. Recio-Fernández ${ }^{c}$, R. Torrens ${ }^{b}$, S. Piñeiro-Hermida ${ }^{b}$, E. Wirthgen ${ }^{d}$, A. \\ Hoeflich $^{d}$
}

anfectious Diseases, Microbiota and Metabolism Unit. Infectious Diseases Department. Center for Biomedical Research of La Rioja (CIBIR). Logroño, Spain.

${ }^{b}$ Lung Cancer and Respiratory Diseases Unit. Center for Biomedical Research of La Rioja (CIBIR). Logroño, Spain. Infectious Diseases, Microbiota and Metabolism Unit. Infectious Diseases Department. Center for Biomedical Research of La Rioja (CIBIR). Logroño, Spain.

${ }^{\mathrm{d} I n s t i t u t e}$ for Genome Biology, Leibniz-Institute for Farm Animal Biology (FBN). Dummerstorf, Germany

Background: Insulin-like growth factors (IGF) and their tyrosine kinase receptor (IGF1R) play pivotal roles in the physiology of vertebrate organisms, by regulating somatic growth, The objective was to analyze the metabolic effects of IGF1R deficiency in a mouse model and to investigate the effects of ageing and a high fat diet in these mutant mice. Methods: UBC-CreERT2, Igf1rfl/fl mutant mice were used to suppress signalling in all adult tissues and Igf1r deletion was postnatally induced with tamoxifen. Two different approaches were carried out: 1) IGF1R mutant mice sacrificed at 13 weeks old and 2) IGF1R mutant mice sacrificed at 12 months old (two tamoxifen administrations to ensure deletion of IGF1R). To test the effects of IGF1R deficiency on obesity, aged-mice were fed with either a standard laboratory diet or a high fat diet for 10 weeks before their sacrifice. Results: Short-term study: mutant mice were insulin-resistant, with high IGF-1 and growth hormone plasma levels. Male mutant mice also presented increased hepatic triglyceride content and an increased expression of SREBP1c gene. This model of IGF1R deficiency reflects the same metabolic alterations observed in patients, and, therefore, could be a useful tool for a deep knowledge of the mechanisms underlying the pathophysiology of this mutation. Long-term study: our results showed a clear metabolic sexual dimorphism dependent on IGF1R and suggest that male mice deficient in this receptor are able to counteract the negative effects induced by ageing and by a high-fat diet in metabolism. In addition, IGF1R deficiency in male aged mice seems to decrease inflammation in adipose tissue. Conflict of interests: None.

14

\section{Oral microbiota associated with gender could drive the glycemic response in saliva samples}

M. Minty ${ }^{a, b}$, P. Loubieres ${ }^{a, b}$, F. Tercé $e^{a, b}$, R. Burcelin ${ }^{a, b}$, V. Blasco-Baque Bd,e $^{c, d}$

anstit. National de la Santé et de la Recherche Médicale, INSERM U1048, I2MC, Toulouse, France

bUniversité Paul Sabatier, Toulouse, France.

'Institut des Maladies Métaboliques et Cardiovasculaires (I2MC), INSERM U1048, Toulouse, France.

¿Université Paul Sabatier III (UPS), Toulouse, France.

${ }^{e} \mathrm{CHU}$ Toulouse, Service d'Odontologie Toulouse, France.

Background: Saliva, besides its many known functions in absorption and digestion, has a great interest in medical diagnosis.It is $s$ an ideal biofluid for studying disease-related biomarkers because of its easy sampling. To test the detection capabilities of saliva, we focused on metabolic diseases, particularly diabetes. 
Objectives: In this work, we provide evidence supporting the idea that studying saliva biomarkers could provide an early diagnosis and subsequent follow-up of systemic diseases such as diabetes. Methods: We measured physiognomical and saliva biological parameters mainly related to lipid metabolism. We have studied two main types of salivary parameter: firstly, levels of metabolites and enzyme activities involved in lipid metabolism, and secondly the nature of the microbiome, by sequence analysis of the microbial $16 \mathrm{~S}$ rRNAs. We have set up a pilot study on 10 men and 10 women of the same age before and after feeding to compare fasting salivary samples between men and women to test the idea that gender may be a key factor for driving the insulin response. Results: Our results show that in the fasted state, cholesterol, triglyceride and free fatty acids in the saliva discriminate between women and men, but not after feeding. On the other hand, when analysed as a whole, salivary lipase and cholesterol mainly discriminate between the fasting and feeding state. In addition, some bacterial species were also modified after feeding. Conclusion: In agreement with other published results, we confirm the growing interest in saliva as a diagnostic biological fluid for metabolic diseasesand we discuss the biological significance of these changes in oral and systemic pathologies, and the potential for their use in oral biosensors in predicting systemic diseases. Conflict of interests: Authors declare no conflicts of interest.

15

\section{Are High Carbohydrate Low Fat (HCLF) diets fine strategies to approach obesity and overweight?}

L. Amezketa García ${ }^{a}$, M. Martínez-Pineda ${ }^{a}$

${ }^{a}$ Nutrition and Food Science Unit. Department of Animal Production and Food Science. Faculty of Health and Sport Sciences, University of Zaragoza, Huesca, Spain.

Background: Nowadays new approaches are being used to body weight control in overweight and obese patients. In the last years, high carbohydrate low fat (HCLF) diets have been proposed as a new alternative strategy in weight management and its related body excess weight parameters prevention. In this type of diets $\leq 20 \%$ of the energy intake comes from fats and $\geq 60 \%$ of the energy intake comes from carbohydrates. Objectives: The objective of the work is to examine the available scientific evidence about how HCLF diet influence in weight management and other health related parameters such body composition, glycemia, insulinemia and inflammatory markers like C-reactive Protein, in healthy overweight (BMI $>25)$ and obese (BMI 30-44) subjects. Methods: A systematic review has been applied. Results: The results obtained have been scarce and only a maximum of 12 months of dietary intervention. The studies showed that HCLF diet seems to be effective in weight management, with a decrease in body weight, between $-1.6 \mathrm{~kg}$ and $-11.3 \mathrm{~kg}$ especially in population with higher BMI and regardless studies duration. Body composition improves, with a reduction in fat mass between $1.4 \%$ to $4.5 \%$. An intake of low glycemic index foods and regular moderate physical activity together with HCLF diet contribute positively to the weight management and body composition. The evidence found indicates that, in short term, this type of diet could be useful in the management of other cardiovascular risk measures related. Other parameters analyzed, as insulin resistance, lipid profile (LDL cholesterol, HDL cholesterol, triglycerides, total cholesterol) and C-reactive protein, have also improved in subjects with this kind of dietary intake. Conclusion: Results are encouraging; however, more studies are needed to clarify the effect of HCLF diets long-term, the evolution of other health indicator parameters, as well as the adherence to this type of diet. Conflict of interests: The authors declare no conflicts of interest.

The delipidating effect of Resveratrol and its metabolites in obese hepatocytes

J. Trepiana ${ }^{a}$, S. Gómez-Zorita ${ }^{a, b}$, E. Irles ${ }^{c}$, ,A. Mosqueda-Solís ${ }^{a}$, MP. Portillo ${ }^{a}$

${ }^{a}$ Nutrition and Obesity group, Department of Nutrition and Food Sciences, Faculty of Pharmacy, University of the Basque Country (UPV/EHU) and Lucio Lascaray Research Centre, Vitoria, Spain.

${ }^{b}$ CIBER Physiopathology of Obesity and Nutrition (CIBERObn), Institute of Health Carlos III, Spain. ${ }^{c}$ CIBER Diabetes and Associated Metabolic Diseases (CIBERdem), Molecular Neuroendocrinology, Principe Felipe Research Center, Valencia, Spain. 
Background: Liver steatosis is considered the hepatic component of the metabolic syndrome associated with obesity and insulin resistance. This condition is characterized by an excessive lipid accumulation in the form of triglycerides and cholesterol in the liver. Due to the low bioavailability of resveratrol, we aimed at investigating whether resveratrol metabolites exert any beneficial effect in steatosis prevention. Methods: An in vitro model of obese hepatocytes was created incubating mouse AML-12 hepatocytes with $0.3 \mathrm{mM}$ of palmitic acid (PA) for $18 \mathrm{~h}$. Resveratrol (RSV) and its metabolites trans-resveratrol-4'-0-glucuronide (R-4G), trans-resveratrol-3-0-glucuronide (R-3G), trans-resveratrol-3-0-sulfate (R-S) and dihydroresveratrol (DH$\mathrm{R}$ ), were co-incubated with PA at 1, 10 or $25 \mu \mathrm{M}$. Cell toxicity, hepatic triglycerides, FAS and MTP activities, DGAT2, UCP2 and p-ACC protein expressions were analyzed. Results: Neither RSV nor its metabolites prevented the toxicity induced by PA during the co-incubation. As it was expected, PA increased triglyceride levels (+92\%) compared with the control cells. Interestingly, RSV and its metabolites $(1 \mu \mathrm{M})$, significantly prevented liver fat accumulation vs PA group. However, at higher concentrations, not delipidating effect was observed, except with R-DH $(10 \mu \mathrm{M})$ incubation. According to that, RSV prevented the rise of FAS activity presented in the PA-induced obese group (RSV $1 \mu \mathrm{M},-62 \%)$. Moreover, RSV $(10 \mu \mathrm{M})$ prevented the MTP activity increase induced by PA. Regarding the protein expression, a tendency to increase UCP2 protein expression was observed with RSV $25 \mu \mathrm{M}$; consequently, RSV could facilitate fatty acid oxidation. No significant changes were observed in DGAT2 and p-ACC protein expressions. Conclusion: The present study shows for the first time the delipidating effect of resveratrol metabolites in the liver. Both, the parent compound and its metabolites are involved in the prevention of hepatic fat accumulation of resveratrol.

Conflict of interests: The authors declare no conflict of interest.

17

\section{Effects of virgin olive oils differing in their bioactive compound contents on postprandial plasma insulin and gastrointestinal hormones in healthy adults.}

MD. Mesa ${ }^{a, b}$, E. Sánchez Rodríguez ${ }^{a}$, S. Biel Glesson ${ }^{d}, J A$. Espejo Calvo ${ }^{d}$, M. Fito ${ }^{e}$, M. Roca ${ }^{f}$, E. Martínez de Victoria ${ }^{a}$, A. Gil ${ }^{a, b}$

${ }^{a}$ Department of Biochemistry and Molecular Biology II, Institute of Nutrition and Food Technology "José Mataix", Biomedical Research Center, University of Granada, Granada, Spain.

${ }^{b}$ Instituto de Investigación Biosanitaria de Granada (ibs.GRANADA), Granada, Spain.

'Fundación Pública Andaluza para la Investigación Biosanitaria de Andalucía Oriental "Alejandro Otero" (FIBAO), Granada, Spain.

dInstituto para la Calidad y Seguridad Alimentaria (ICSA), Granada, Spain.

eSpanish Biomedical Research Networking Centre, Physiopatology of Obesity and Nutrition (CIBERObn), Madrid, Spain. Cardiovascular Risk and Nutrition Research Group, Barcelona, Spain.

fFood Phytochemistry Department, Instituto de la Grasa, Consejo Superior de Investigaciones Científicas (CSIC), University Campus Pablo de Olavide, Sevilla, Spain.

Background: The present work is part of the NUTRAOLEUM study aimed to evaluate the healthy effect of virgin olive oils (VOOs) enriched with phenolic and triterpenes compounds from the olive fruit on healthy adults. Objectives: Here we present postprandial data of plasma insulin and selective hormones related to the control of food intake. Methods: Eighteen subjects (20-50 years) from the general population of Granada (Spain), were selected for the postprandial assay, and randomly assigned to a crossover, controlled, double-blind, intervention. After a 2-wk washout period, subjects received (30 mL) of three oils: 1) a control VOO obtained by traditional procedures (124 ppm of phenolic compounds and 86 ppm of triterpenes); 2) an optimized VOO (OVOO) (490 ppm of phenolic compounds and $86 \mathrm{ppm}$ of triterpenes); and 3) a functional olive oil (FOO) high in phenolic compounds (487 ppm) and enriched with triterpenes (389 ppm). The oil was accompanied with a standard piece of bread $(80 \mathrm{~g})$. Blood samples were taken before $(0 \mathrm{~h})$ and after the oils intake, at 30, 45, 60, 120, 240 and 360 minutes. Plasma gastrointestinal hormones and insulin were determined by the xMAP technology (Luminex). Postprandial area under the curve (AUC) was calculated. Results: The postprandial release of gastric inhibitory polypeptide was higher after the intake of the control VOO than after the OVOO ( $\mathrm{p}=0.044)$, and a tendency of lower insulin release was observed after the intake of FOO compared to control VOO $(\mathrm{p}=0.055)$. The AUCs for glucagon like peptide-1, ghrelin, pancreatic polypeptide and peptide YY were similar after the intake of the three olive oils. Conclusions: The enrichment of olive oil in its own bioactive compounds seems to improve the postprandial response in relation to a control VOO with low amounts of bioactive compounds in healthy subjects. (Clinical Trials NCT02520739). Conflict of interests: The authors declare no conflicts of interest. Acknowledgement: The 


\title{
Acting on key-factors of long-term weight maintenance: physical rehabilitation programs at the Clinique du Château de Vernhes
}

\author{
M. Despeaux ${ }^{a}$, M. Bonnefoy ${ }^{b}$, C. Rusques ${ }^{b}$, M. Boulanger ${ }^{b}$, D. Guillard ${ }^{b}$, J. Alvarez ${ }^{b}$, F. Sanguignol $^{a}$ \\ aUnité Recherche. Clinique du Château de Vernhes. Bondigoux. France. \\ ${ }^{b}$ Capacity. Clinique du Château de Vernhes. Toulouse. France.
}

Background: obesity is a pandemic disease causing premature death and severe morbidity. One of the biggest challenges in obesity management is weight-relapse following an initial weight-loss program. Patient education and physical activity are key factors in long-term weight maintenance. We aimed to evaluate whether physical rehabilitation training could decrease weight-relapse and enhance physical abilities. Objectives: To evaluate how effective can physical rehabilitation weight-relapse prevention programs be with obese patients. We assessed the benefits of a 4 months ambulatory physical rehabilitation program on body composition, on cardiovascular and physical performances. Methods: The physical rehabilitation program was a prospective, observational, descriptive cohort study. A 4 months physical rehabilitation program was negotiated with the patients, in accordance with the joint educational diagnosis needs that were identified. Anthropometric (weight, waist size and impedancemetry), cardiovascular (blood pressure, heart rate and V02max) and physical evaluation (3RM strength score, flexibility test and endurance test) were performed on inclusion and at end-point. Results: $74.4 \%$ of the 42 rehabilitation participants completed the program. Mean age was 57.6 y.o. ( \pm 11.9 ), $59 \%$ were men and all patients were obese. After 4 months, waist-size and body fat-mass were significantly reduced $(-5.1 \mathrm{~cm} \pm 4.2, \mathrm{P}<0.001 ;-5.2 \% \pm 4.9$, $\mathrm{P}<0.001)$ but weight was unchanged $(-.9 \mathrm{~kg} \pm 18.2, \mathrm{P}=0.722)$. Systolic $(-7.0 \mathrm{mmHg} \pm 10.0, \mathrm{P}<0.001)$ and diastolic $(-3.6 \mathrm{mmHg} \pm 6.9, \mathrm{P}<0.001)$ blood pressure were decreased, as well as resting heart rate $(-5.2 \mathrm{bpm}$ $\pm 8.1, \mathrm{P}<0.001)$ and VO2max $(6.4 \mathrm{~mL} / \mathrm{min} / \mathrm{kg} \pm 5.9, \mathrm{P}=0.003)$. Similarly, strength $(+6.1 \mathrm{~kg} \pm 4.1, \mathrm{P}<0.001)$, flexibility $(0.3 \mathrm{~cm} \pm 0.7, \mathrm{P}<0.001)$ and endurance $(19,83 \mathrm{sec} \pm 23,24, \mathrm{P}<0,0001)$ were enhanced. Conclusion: patients with obesity may improve abdominal fat-mass loss, cardiovascular and physical capacities when undergoing a 4 month ambulatory tailored physical activity program based on educational diagnosis.

Conflict of interests: No conflicts of interest.

19

\section{Studying the food preferences after bariatric surgery: The BARIATASTE pilot study}

E. Guyot ${ }^{a}$, S. Iceta ${ }^{b}$, J.A. Nazare ${ }^{c}$, A. Dougkas ${ }^{d}$, E. Disse $^{e}$,

${ }^{a}$ Center for Research in Human Nutrition Rhône-Alpes, Institut Paul Bocuse Research Center, Univ Lyon, CarMeN Laboratory, INSERM, INRA, INSA Lyon, Université Claude Bernard Lyon 1. Pierre-Bénite, France.

${ }^{b}$ Referral Center for Eating Disorder, Hospices Civils de Lyon, PSYR2 - Psychiatric disorders: from Resistance to Response, Université Claude Bernard Lyon 1. Bron, France.

'Center for Research in Human Nutrition Rhône-Alpes, Univ Lyon, CarMeN Laboratory, INSERM, INRA, INSA Lyon, Université Claude Bernard Lyon 1. Pierre-Bénite, France.

dInstitut Paul Bocuse Research Center. Ecully, France.

eIntegrated Center for Obesity, Department of Endocrinology and Diabetes, Hospices Civils de Lyon, CarMeN Laboratory, Université Claude Bernard Lyon 1. Pierre-Bénite, France.

Background: The most effective treatment for obesity is bariatric surgery. However, nutritional complications are common and a weight regain may occur in almost $20 \%$ of the patients. Clinical experience and scientific literature suggest changes in dietary choices and preferences after bariatric surgery, which may contribute to the weight loss and/or the weight regain. Yet, there is inconsistency among the studies as they do not use objective measures to assess food preferences and few compare the different types of surgical technics. Our objective is to compare the liking and wanting of consuming certain foods varying in composition and appearance, among patients with a sleeve gastrectomy and a gastric bypass. Methods: In a cross-sectional clinical trial at the Integrated Center for Obesity of Lyon, 90 patients were divided into three 
groups: a control group of patients with unoperated severe obesity (OB), a group of patients with a sleeve gastrectomy (LSG) and a group of patients with a gastric bypass (RYGB) 6-24 months post-surgery. We assess food preferences using the Leeds Food Preference Questionnaire (LFPQ), a behavioral computer task. We also assess binge eating, impulsivity and food addiction with self-report questionnaires. Results: Preliminary analysis showed a significant difference in liking scores, with the OB group having higher scores for high fat savory (HFSA), high fat sweet (HFSW) and low fat sweet foods (LFSW) ( $<<0.0001)$ relative to LSG and RYGB groups. Additionally, LSG had greater score of liking for HFSA than RYGB ( $p=0.001)$. There was a high correlation between the binge eating score and the liking scores for HFSA, HFSW and LFSW ( $\mathrm{p}<$ 0.001). Our results increase the knowledge regarding changes in food preferences after bariatric surgery. Conclusion: This pilot study will be followed by a prospective study using the LFPQ and an experimental restaurant to assess eating behavior in an ecological setting. Conflict of interests: The authors declare that there is no conflict of interest.

20

\title{
The role of untargeted metabolomics in food consumption biomarkers identification
}

\author{
L. Lacalle-Bergeron ${ }^{a}$, J.V. Sancho ${ }^{a}$, F.J. López ${ }^{a}$, F. Hernández ${ }^{a}$, C. Ortega-Azorín ${ }^{b, c}$, E.M. Asensio ${ }^{c}$, O. Coltell, ${ }^{c, d}$ \\ ${ }^{a}$ Research Institute for Pesticides and Water (IUPA), University Jaume I, Castellón de la Plana, Spain. \\ ${ }^{b}$ Department of Preventive Medicine, University of Valencia, Valencia, Spain. \\ ${ }^{\mathrm{C} C I B E R}$ Fisiopatología de la Obesidad y Nutrición (CIBERObn), Instituto de Salud Carlos III, Madrid, Spain. \\ ${ }^{\mathrm{d}}$ Department of Computer Languages and Systems, University Jaume I, Castellón de la Plana, Spain
}

Background: Diet is one of the most important lifestyle factors associated with health status. However, the common observational approaches for data collection may contain several recall bias that may significantly affect the sequent associations found. Metabolomics can be applied to discover new Biomarkers of food intake (BFIs) with the final goal to provide a more objective food consumption measurements, a complete picture of the dietary intake and help to unravel the molecular mechanisms whereby diets affects positively or negatively to health.New and powerful analytical technologies like ion mobility spectrometry coupled to high resolution mass spectrometry (IMS-HRMS) provide new tools to facilitate the complicated task of biomarkers elucidation. Objectives: Identification of short-term and long-term biomarkers of orange consumption in plasma samples based on the metabolomic profile. Evaluation of the potential of the IMSHRMS in untargeted metabolomics. Methods: Plasma samples were obtained for an acute (4h after consumption) and a medium-term intervention study (after 1 month of continued intake). The crossover, randomized trial enrolled 30 healthy participants (aged 25 \pm 2.8 ). Plasma samples were collected at baseline (after fasting), and at $4 \mathrm{~h}$ postprandial. The samples were injected in UHPLC-IMS-QTOF MS. Results: Up to the moment, compounds as stachydrine, betonicine, $\mathrm{N}$-methyltyranine-O-sulfate, synephrime-O-sulfate and $\mathrm{N}$-methyl-proline have been tentatively identified as markers of acute orange consumption. Among them, stachydrine and betonicine have been also identified in the 1-month intervention study. Conclusions: IMSHRMS has demonstrated to be a powerful advance in the elucidation step in metabolomics, providing an additional molecular descriptor, the CCS. Some biomarkers were already reported as coming from the citrus, validating the results obtained. As future works, there is a need for final identity confirmation of the BFI of acute orange consumption using a reference standard. Regarding the chronic study, the elucidation of the potential endogenous biomarkers is still in process. Conflict of interests: The authors declare that there is no conflict of interest.

21

\section{Associations between breast milk hormones and infant anthropometry at four months of age}

B. Pastor Villaescusa ${ }^{a}$, M. Grunewald ${ }^{a}$, ML. Mearin ${ }^{b}$, B. Koletzko ${ }^{a}$, H. Demmelmair ${ }^{a}$

${ }^{a}$ Dr. von Hauner Children's Hospital, LMU-Ludwig-Maximilians-Universität München. Division of Metabolic and Nutritional Medicine, Munich, Germany.

beiden University Medical Center, Department of Pediatrics, Leiden, The Netherlands. 
Background: Breastfeeding is recommended as the optimal infant feeding, providing a wide variety of compounds that are not present in infant formulas, and could play relevant roles in early growth. However, there are controversial findings about the associations of human milk hormones with infant growth during the first months of life. Objectives: We aimed to determine the association of milk adiponectin, leptin, insulin, and IGF-II with infant anthropometry at the age of 4-month. Methods: 144 pairs of mothers and infants participating in the PreventCD trial were studied. Adiponectin, leptin and insulin concentrations in human milk were determined by ELISA, and IGF-II by RIA. Associations were analyzed with multiple linear regressions stratified by sex. The infant length, weight and BMI were introduced as dependent variables, and the milk hormones (insulin, adiponectin, leptin, and IGF-II) were included as predictors, with the exact age (days) and feeding mode (exclusive or mixed breastfeed). P-value $<0.025$ was considered significant (Bonferroni correction). Results: Adiponectin values were significantly higher in milk for female than for male infants $(\mathrm{P}=0.019)$. In females, $15.6 \%$ of the length variance was explained by the predictive model, with a significant association between IGF-II and length $(\beta=0.298, P=0.009)$. Moreover, insulin and adiponectin showed significant associations with infant weight in the model $(\beta=27.3, P=0.005 ; \beta=-43.9, P=0.005$, respectively). The model explained $24.4 \%$ of infant weight variance. IGF-II was the only variable showing a significant association with BMI (means ratio $=0.1, \mathrm{P}=0.015$ ) and explaining $9 \%$ of the outcome. In males, no associations were found. Conclusion: Our data suggest that milk insulin and adiponectin could influence the infant weight in females, and milk IGF-II levels might be relevant for the female infant length at 4-month. Conflict of interests: The authors declare that there is no conflict of interest.

\title{
Sodium/potassium ratio and blood pressure levels in Spanish schoolchildren according to the presence of family history of hypertension
}

\author{
E. Cuadrado-Soto ${ }^{a}$, A.Peral-Suárez ${ }^{a}$, LM. Bermejo-López ${ }^{a, b}$, E. Rodruíguez-Rodríguez ${ }^{b, c}$, P. Sánchez- \\ Rodruíguez ${ }^{a}$, RM. Ortega ${ }^{a, b}$, A. Aparicio ${ }^{a, b}$, AM. López-Sobaler ${ }^{a, b}$.
}

\author{
aDepartment of Nutrition and Food Science, Faculty of Pharmacy, Complutense University of Madrid, \\ Madrid, Spain. \\ ${ }^{\mathrm{b}}$ Research Group VALORNUT-UCM (920030), Faculty of Pharmacy, Complutense University of Madrid, \\ Madrid, Spain. \\ 'Department of Chemistry in Pharmaceutical Sciences, Analytical Chemistry, Faculty of Pharmacy, \\ Complutense University of Madrid, Madrid, Spain
}

Background: Family history of hypertension (h-HT) is associated with an increased risk of high levels of blood pressure (BP) in childhood. In addition to the genetic component, several studies have shown a relationship between sodium and potassium intake and BP. The WHO recommends a urinary sodium-topotassium molar ratio of 1.0. Objectives: To determine the sodium/potassium ratio and the levels of BP in Spanish schoolchildren according to the presence of h-HT. Methods: 289 schoolchildren (134 girls) aged 711 years from five Spanish provinces were studied. Parents completed a questionnaire with health information. Children with h-HT (at least one of the parents had hypertension) were compared to those with no history of hypertension (nh-HT). Children BP was measured with an OMRON Monitor Tensiometer. Sodium and potassium were assessed through 24-hour urine samples. The sodium/potassium ratio was calculated by dividing urinary sodium by potassium expressed as $\mathrm{mmol} / \mathrm{mmol}$. For statistical analysis, Student's t-test, Mann Whitney's U test and Z test were used. Results: The mean sodium/potassium ratio was $2.79 \pm 1.13$. Schoolchildren with $\mathrm{nh}-\mathrm{HT}(\mathrm{n}=263)$ had a lower sodium/potassium ratio $(2.75 \pm 1.06$ vs. $3.24 \pm 1.61 ; \mathrm{p}<0.05)$ and DBP $(67.05 \pm 10.28$ vs. $70.73 \pm 7.22 \mathrm{mmHg} ; \mathrm{p}<0.05)$ than children with $\mathrm{h}-\mathrm{HT}(\mathrm{n}=26)$, without differences in SBP. Girls with nh-HT had lower levels of DBP than those with h-HT (67.38 \pm 10.20 vs. $72.41 \pm 7.21 \mathrm{mmHg} ; \mathrm{p}<0.05)$. Boys with nh-HT had lower levels of SBP than boys with h-HT $(102.7 \pm 11.9$ vs. $109.6 \pm 11.2 \mathrm{mmHg} ; \mathrm{p}<0.05$ ). Also $80 \%$ of boys with $\mathrm{h}-\mathrm{HT}$ had a sodium/potassium ratio above 3 compared to $44.3 \%$ of boys with no h-HT $(\mathrm{p}<0.01)$ and no differences were found in girls. Conclusions: Children with hHT showed higher sodium/potassium and BP levels, which could be related to an inadequate parents intake of sodium and potassium. Boys with h-HT could be especially affected by a higher sodium/potassium ratio. Conflict of interests: The authors declare that there is no conflict of interest. 


\title{
Relationship between Physical Activity Level and Healthy Eating Index in Spanish schoolchildren: sex differences
}

\author{
A. Peral-Suárez ${ }^{a}$, E. Cuadrado-Soto ${ }^{a}$, L.M. Bermejo-López ${ }^{a, b}$, A.M. López-Sobaler ${ }^{a, b}$, A.M. Lorenzo-Mora ${ }^{a}, B$. \\ Navia $^{a, b}$, J.M. Perea-Sánchez ${ }^{c}$, R.M. Ortega ${ }^{a, b}$
}

${ }^{a}$ Departmen of Nutrition and Food Science. Faculty of Pharmacy at Complutense University of Madrid. Madrid, Spain.

${ }^{\mathrm{b}}$ Research group VALORNUT-UCM (920030). Faculty of Pharmacy at Complutense University of Madrid. Madrid, Spain.

${ }^{\mathrm{C}}$ Faculty of Health Science. University Alfonso X El Sabio. Madrid, Spain.

Background: Adopting a healthy lifestyle since childhood have an important role in preventing overweight and obesity and derived diseases in the future. This healthy lifestyle includes the practice of physical activity and a properly balanced diet. Objective: The aim of the study was to relate the physical activity practice of schoolchildren with their diet quality. Methods: A sample of 359 children (178 girls) aged 7-11 years from 11 schools of 6 Spanish provinces was studied. A daily physical activity questionnaire was used to obtain their Physical Activity Level (PAL). Children were divided into two groups depending on their PAL using the median as a cut-off point: Low activity (LA) (PAL $\leq 1.44$ ) or high activity (HA) (PAL>1.44). To assess the diet quality, the children completed a 3-days food record with their parents help, including one weekend day. DIAL software was used to process this questionnaire and estimate macronutrients intake and Healthy Eating Index (HEI). For statistical analysis, Student's T test and Mann Whitney's U test (non-parametrical variables) were used to compare means, and Spearman's Rho was used for correlations. Results: Boys presented higher PAL value than girls $(1.46 \pm 0.07$ vs. $1.44 \pm 0.07 ; \mathrm{p}<0.05)$. The higher PAL in girls the higher HEI $(r=0.188 ; p<0.05)$. Furthermore, HA girls had higher HEI $(59.80 \pm 9.23$ vs. $56.32 \pm 10.03 ; p<0.05)$ and consumed less energy from fats $(39.35 \pm 4.52 \%$ vs. $40.84 \pm 4.84 \%$; $<<0.05)$ and saturated fats $(13.25 \pm 1.90 \%$ vs. $14.07 \pm 2.28 \%$; $\mathrm{p}<0.05$ ) than LA girls. These results were not found in boys. Conclusions: Girls are less active than boys. However, more active girls follow a healthier diet than those less actives. This relationship was not shown in boys. These results show the need of personalized strategies of nutritional education focus on sex differences. Conflict of interests: The authors declare no conflicts of interest. Acknowledgement: Financed by Complutense University of Madrid through projects PR6/13-18866, GR58/08, GR3/14, GR15/17 and FEII16/127.

\section{4}

\section{Involvement of microRNAs in the improvement of glycaemic control induced by a grape seed extract in patients with metabolic syndrome}

\author{
A. Léniz Rodríguez ${ }^{a, b}$, D. Martínez-Maqueda ${ }^{c}$, A. Fernández-Quintela $^{b, d}$, J. Pérez-Jiménez ${ }^{c}$, M.P. Portillo ${ }^{b, d}$ \\ ${ }^{a}$ Araba Integrated Health Care Organization. Basque Health Service (Osakidetza), Araba, Spain \\ ${ }^{b}$ Nutrition and Obesity Group, Department of Nutrition and Food Science, Faculty of Pharmacy and Lucio \\ Lascaray Research Center, University of the Basque Country (UPV/EHU), Vitoria, Spain. \\ 'Department of Metabolism and Nutrition, Institute of Food Science, Technology and Nutrition (ICTAN- \\ (SIC), Madrid, Spain. \\ ${ }^{d}$ CIBER Fisiopatología de la Obesidad y Nutrición (CIBERObn), Instituto de Salud Carlos III (ISCIII), Madrid, \\ Spain.
}

Background: The metabolic syndrome (MS) is characterized by hypertension, high circulating glucose and triglyceride levels, low high-density lipoprotein levels and abdominal obesity. It affects up to $30 \%$ of adult population in western countries and it is an important determinant for the appearance of type 2 diabetes or cardiovascular diseases. In several pre-clinical studies, grape polyphenols have demonstrated to improve insulin sensitivity, which is one of the main alterations appearing in MS. Interestingly, microRNAs (miRNAs) are small(19-25 nucleotide length) non-coding RNAs that bind to the untranslated region of their target mRNAs modifying their expression. They can be found in peripheral blood and they are known to play a key role in glucose metabolism. Objectives: The objective of our study is to analyze whether the positive response in glycaemic control observed in patients with metabolic syndrome treated with a grape seed 
extract is mediated by circulating miRNAs. Methods: 49 subjects aged 18-70 years and diagnosed with MS were recruited. Their usual diet was supplemented with $8 \mathrm{~g} /$ day dried and milled grape pomace. Blood samples were collected after 6 weeks of treatment, and plasma was obtained by centrifugation at $1000 \mathrm{~g}$ for 15 min. The fasting glucose and insulin concentrations were measured by using commercial kits. A decrease after treatment of $10 \%$ was taken as reference to classify subjects between responders and non-responders. The miRNA profiling of both groups was assessed by next generation sequencing, and the expression of circulating miRNAs was confirmed by qRT-PCR. Statistical analysis was performed using SPSS v23. Results: A significant decrease in insulin levels after treatment $(8.5 \mathrm{vs} 5.5 \mu \mathrm{U} / \mathrm{mL})$ was observed, without changes in glucose levels. Before supplementation,miR-30c-5p,miR-23a-3p,miR-222-3p,miR-let7a-5p,miR-151a5p,miR-181a-5p and miR-10a-5p were shown to be differentially expressed between responders and nonresponders. After qRT-PCR analysis, statistically significant differences in miR-222-3p were observed, as well as a tendency in miR-let7a-5p with higher expressions in the responder group. Conclusions: The obtained results suggest that the beneficial effects of grape polyphenols on glucose metabolism could be mediated by microRNAs, in particular by miR-222-3p. Conflict of interests: The authors declare no conflicts of interest.

\title{
Validity of a choice-modeling Google-form questionnaire to implement Exercise is Medicine initiative by a multidisciplinary lifestyle team in healthcare settings
}

\author{
S. Calonge-Pascual ${ }^{a, b}$, F. Fuentes-Jiménez ${ }^{a, b}$, F. Novella-María-Fernández ${ }^{a, c, d}$, D. Villalvilla-Soria ${ }^{e}$, J.A. \\ Casajús-Mallen $^{b, f, g}$, R. Arnal-Selfa ${ }^{h}$, S. Belmonte-Cortés ${ }^{i}$, M. González-Gross ${ }^{a, b, g}$
}

${ }^{a}$ ImFINE Research Group, Department of Health and Human Performance, Faculty of Physical Activity and Sport Sciences-INEF. Universidad Politécnica de Madrid, Spain.

${ }^{b}$ Red Española de Investigación en Ejercicio Físico y Salud en Poblaciones Especiales (EXERNET), Spain

${ }^{c}$ Centro de Salud Rosa Luxemburgo. San Sebastián de los Reyes, Spain.

dUniversidad Europea de Madrid, Spain.

${ }^{e}$ Centro de Salud Alcalá de Guadaira, Spain.

fGENUD Research Group. Department of Physiatrist and Nursing, Faculty of Health Sciences. University of Zaragoza, Zaragoza, Spain.

${ }^{9}$ Biomedical Research Centre of Physiopathology of obesity and nutrition, CIBERObn, B12/03/30038. Carlos III Health Institute, Spain.

hDirección Asistencial Enfermería DA Norte. Gerencia Asistencial de Atención Primaria. Comunidad de Madrid, Spain.

'Dirección General de Salud Pública. Consejería de Sanidad. Comunidad de Madrid, Spain.

Background: Physical inactivity and unhealthy dietary lifestyles are two leading morbi-mortality risk factors. In contrast, healthy lifestyles are a cost-effective resource trying to be implemented by different worldwide strategies. Objectives: To validate a choice-modeling Google-form questionnaire and to assess the self-perception of Primary healthcare (PHC) staff in regard to the inclusion of nutritionists and sports scientists as health providers in PHC of the Region of Madrid. Methods: A Google-form questionnaire was developed following a previous content-analysis processing of two verbatim transcribed focus group sessions. Once designed, the questionnaire was sent to 20 experts for validation. 10 nurses and 10 sports medicine physicians answered 30 questions in a 1-5 points Likert scale. Aiken's $V$ coefficient values $>0.75$ were considered to validate each item, using Visual Basic 6.0 software. Afterward, the questionnaire was sent to all Madrid PHC nurses and physicians via email. Pearson's chi squared test analysis was performed with IBM SPSS statistics software. Results: All 10 sports medicine physicians and 10 nurses, with a mean of $28.40 \pm 5.10 \mathrm{y}$ and $16.3 \pm 11.8 \mathrm{y}$. experience in public health promotion, respectively, validated the questionnaire ( 0.77 to 1.00 Aiken-V values). The firsts 258 physicians ( 198 females) and 253 nurses (222 females) who answered anonymously had a mean of $22.27 \pm 8.73 \mathrm{y}$. and $15.35 \pm 9.51 \mathrm{y}$ of PHC experience, and agreed practically in total to the health benefits of physical activity. PHC professionals were willing to collaborate in physical activity promotion with sports scientists (61.60\%) and nutritionists (60.90\%). Besides, PHC nurses and physicians also agreed to collaborate with sports scientists (64.20\%) and nutritionists $(56.00 \%, \mathrm{P}<0.05)$ for exercise prescriptions, as a multidisciplinary PHC team. Conclusions: The results of this study can help to implement healthy-lifestyle promotions in the national PHC System 
according to the Exercises is Medicine initiative. Conflict of interests: The authors declare no conflicts of interest.

\title{
Relationship between physical exercise, obesity and the risk of depression in the general population: Results from the PISMA-ep study
}

\author{
M. Rivera ${ }^{a}$, A. Porras-Segovia ${ }^{b}$, D. López-Chávez ${ }^{c}, E$. Molina $^{d}$, B. Gutiérrez ${ }^{e}$, J. Cervilla $^{e}$ \\ aDepartamento de Bioquímica y Biología Molecular II, Facultad de Farmacia, Universidad de Granada, \\ Granada, España. \\ ${ }^{\mathrm{b}} \mathrm{H}$. U. Fundación Jiménez Díaz Madrid, España. \\ ${ }^{\mathrm{C}}$ Facultad de Medicina, Universidad de Granada, Granada, España. \\ ${ }^{d}$ Facultad de Ciencias de la Salud, Universidad de Granada, Granada, España. \\ eDepartamento de Psiquiatría, Facultad de Medicina, Universidad de Granada, Granada, España.
}

Background: Depression is one of the most prevalent and disabling mental disorders. Sedentarism and obesity are recognized risk factors for depression. Physical exercise has shown beneficial effects on mental health and there is an increasing awareness of its potential as a therapeutic and preventive tool for depression. No epidemiological studies have explored the role of physical activity and obesity as potential correlates of depression in the Spanish population. Objectives: i) to explore the association between depression and two strongly linked variables (physical exercise and obesity), and ii) to test the possible influence that the intensity of the physical exercise may have on depression. Methods: The PISMA-ep is a cross-sectional community-based study of mental disorders based on a sample of 4,507 individuals representative of the adult population of Andalusia. A diagnosis of depression was ascertained using the MINI interview. The information on physical exercise covers the type of exercise and its intensity. We tested the association between body mass index (BMI) and BMI categories, and depression. The association between depression and physical exercise was tested comparing the frequency of physical exercise between individuals with depression and controls, and testing if there was a linear association between depression and an increasing level of intensity of exercise. Results: Increases in BMI and obesity were significantly associated with depression. The practice of exercise was associated with a lower rate of depression showing a decrease of $40 \%$ in the frequency of depression in participants who practise exercise. We also found a lineal significant association showing the greatest intensity of exercise and the lowest probability of depression. Conclusions: Depression is associated with higher BMI and reduced physical activity. Besides it shows the importance of exercise intensity, so that at higher intensity, less presence of depression. This study also highlights the importance of including physical exercise in the treatment of depression. Conflict of interests: The authors declare no conflicts of interest.

\section{Relationship between body composition, nap and night-time sleep in people aged 65 and over}

\author{
D. Escobar-Toledo ${ }^{a, b}$, E. Gesteiro ${ }^{a, b}$, A. Gómez-Cabello ${ }^{c}, J$. Jiménez ${ }^{a, b}$, S. Vila-Maldonado ${ }^{d, e}$, J. Pérez-Gómez ${ }^{f}$, \\ G. Villa-Vicente ${ }^{c, g}$, I. Ara-Royo ${ }^{d, h}$, M. González-Gross ${ }^{a, b, g}$
}

amFINE Research Group, Department of Health and Human Performance, Faculty of Physical Activity and Sport Sciences-INEF. Universidad Politécnica de Madrid, Spain.

${ }^{\mathrm{b}}$ Red Española de Investigación en Ejercicio Físico y Salud en Poblaciones Especiales (EXERNET), Spain.

'GENUD Research Group. Department of Physiatrist and Nursing, Faculty of Health Sciences. University of Zaragoza, Zaragoza, Spain.

${ }^{\mathrm{d}}$ GENUD (Growth, Exercise, Nutrition and Development) research group. Universidad Castilla La Mancha. Toledo. España.

eBiomedical Research Networking Center on Frailty and Healthy Aging (CIBERFES). Madrid. España.

fDepartamento de Didáctica de la expresión musical, plástica y corporal. Universidad de Extremadura. Badajoz. España. 
${ }^{9}$ Biomedical Research Centre of Physiopathology of obesity and nutrition, CIBERObn, B12/03/30038

${ }^{h}$ Facultad de Ciencias del Deporte. Universidad de Castilla-La Mancha. Toledo. España.

Background: Sleep habits are strongly linked to health during whole life (1). However, nap habit is hardly ever considered despite it is strongly rooted in the Mediterranean lifestyle. The last scientific findings show that short naps could be beneficial for general population (2). Otherwise, older people used to suffer from more somnolence and sleep disorders (3), therefore naps could be a symptom of disease instead of a healthy habit. Objectives: To assess the relationship between sleeping habits and fat mass (FM) in people aged 65 and over, focusing on nap habits. Methods: 829 (75,1\% female) elders from the third cross-sectional data gathering of the EXERNET-Elder 3.0 cohort were analyzed. The participants filled a validated questionnaire whereas body composition was assessed through bioimpedance and anthropometry. ANOVA and linear regression analysis were used to quantify the relationship between FM and sleep habits. Nap duration was divided by intervals $\left(0^{\prime}, 1^{\prime}-30^{\prime}, 31^{\prime}-60^{\prime},>60^{\prime}\right)$. Results: \%FM, body mass index (BMI) and waist circumference (WC) were different according to duration of nap in men but only \%FM in women $(\mathrm{p}<0.05)$. No differences were found regarding hip circumference (HC). The linear regression was corrected by age, physical activity habits, adherence to Mediterranean diet, time walking, diseases, medication and quantity and quality of night-time sleep. Even then, the differences were still significant in men but not in women (\%FM: $\beta=0.209$, $p=0.008$; IMC: $\beta=0.178, p=0.018$; WC: $\beta=0.210, p=0.004$ ), meaning that the longer the nap, the greater the FM in man. Older people who nap more than $1 \mathrm{~h}$ present higher FM compared to those referring shorter nap time $(\mathrm{p}<0.05)$. Conclusions: Our data show that short naps are not related to higher \%FM. However, older people who nap more than $1 \mathrm{~h}$ present higher FM compared to the other groups in every estimation. This means that could be a threshold as nap should be considered as a risk factor. Conflict of interests: The authors declare no conflicts of interest.

\title{
Inhibition of glucolipotoxicity-induced metabolic stress by metabolites from Hibiscus sabdariffa on hypertrophied 3T3-L1 adipocytes
}

\author{
M. Olivares-Vicente ${ }^{a}$, E. Roche ${ }^{b, c}$, M. Herranz-López ${ }^{a}$, V. Micol ${ }^{a}$
}

anstituto de Investigación, Desarrollo e Innovación en Biotecnología Sanitaria de Elche (IDiBE) and Instituto de Biología Molecular y Celular (IBMC). Miguel Hernández University (UMH). Elche. Spain.

${ }^{b}$ Department of Applied Biology-Nutrition. Institute of Bioengineering. Miguel Hernandez University, Alicante, Spain.

Institute for Health and Biomedical Research (ISABIAL-FISABIO Foundation). Alicante. Spain.

Introduction: Recent evidences show that polyphenols from Hibiscus sabdariffa (HS) may have relevant applications in diseases associated to obesity. These data suggest that the extract may modulate pathways related to energy management and inflammation, improving metabolic disturbances associated to obesity. Nevertheless, although there seems to be synergistic interactions between its components, compounds or metabolites responsible for such effects are still unknown. Objectives: Our aim was to study the effect of a polyphenol-enriched HS extract (PEHS) and several polyphenols and metabolites derived from HS on oxidative stress, triglyceride accumulation, inflammation and the expression of proteins that regulate energy metabolism in 3T3-L1 adipocytic cell line and pancreatic $\beta$-cells under glucotoxicity and lipotoxicity conditions. Methods: Oxidative stress and triglyceride content were determined by using fluorescent dyes, inflammation was determined by measuring secreted adipokines by ELISA and expression of metabolic proteins were analyzed by Western blot. Results: Our result highlighted that quercetins (quercetin and quercetin-3-glucuronide) could be the main responsible of bioactive effects of the whole PEHS extract to revert the glucolipotoxicity-induced metabolic stress in adipose tissue. Specifically, quercetin-3-glucuronide, which is a quercetin metabolite, was the more active compound among all the quercetins tested and it is postulated that it mediates these effects through modulation of proteins involved in energy and lipid metabolism such as AMPK, FASN and PPAR- $\alpha$. Conclusions: These findings may facilitate subsequent develop of polyphenolic mixtures from HS which can be used with greater guaranties in clinical and applied studies focusing on obesity and related disorders. Conflict of interests: The authors declare no conflicts of interest. Acknowledgement: This work was supported by the project AGL2015-67995-C3-1-R from Spanish Ministry of Economy and Competitiveness; scholarships ACIF/2016/230 and APOSTD/2017/023 from 
Generalitat Valenciana cofinanced with the European Social Fund; and CIBER (CB12/03/30038, Fisiopatologia de la Obesidad y la Nutricion, CIBERobn, Instituto de Salud Carlos III).

The authors María Herranz-López and Vicente Micol share author co-seniorship.

29

\title{
Spanish food composition database: presentation of the new implemented interface and the challenges in progress
}

\author{
M.A. Martínez Burgos ${ }^{a, b}$, A. Moreno Martínez ${ }^{b}$, I. Martínez-Victoria Carazo ${ }^{b}$, M.D. Yago Torregrosa ${ }^{a, b}$, M. \\ Mañas Almendros ${ }^{a, b}$, E. Martínez-Victoria Muñoz ${ }^{a, b}$ \\ ${ }^{a}$ Department Physiology, Faculty of Pharmacy. University of Granada, Granada, Spain. \\ ${ }^{b}$ Institute of Nutrition and Food Technology "José Mataix" (INYTA). Center for Biomedical Research \\ (CIBM). University of Granada, Granada, Spain.
}

Background: The food composition databases (BDCAs) are an integral part of nutrition and health, research, patient treatment, manufacturing processes and consumer information (Finglas et al., 2014). Food Composition Data (DCAs) can be obtained from different sources. There are several proposals to evaluate to evaluate its quality (Martínez-Victoria et al., 2015). BEDCA is incorporated into the EuroFIR-AISBL, through FoodExplorer, an innovative interface for DCAs, which allows users to search for information from a wide range of data, linking food and nutrients from different BDCAs. Objectives: Implementation of a new version of BEDCA with data quatity: Incorporation of new foods and components. Recipe and ration calculation systems. Coding of new foods with two standardised food classification and description systems: FoodEx2 (EFSA 2017) and LanguaL (version 2008). Sustainability parameters: water, carbon and ecological footprint. Nutritional services: Software. Support for food companies. Methods: FoodEx2 (EFSA 2017) and LanguaL (version 2008). Scientific literature. Excel. BEDCA (version 2.2). SPSS (version 22.0). Results: Inclusion, at the new version of BEDCA of: 950 foods, 48 Components: Macro and micronutrients, "Lost values" of micronutrients, Individual and trans fatty acids, Mono and disaccharides, Non-provitaminic carotenoids, List of foods with natural or added sugar, 310 analytical methods and associated references, Food classification with LanguaL and FoodEx2, Gluten-free food data collection, Inclusion of BEDCA in EVALFINUT, Analytical data of meat's industry, Data of content in FODMAPs of foods, Elaboration of a protocol of sustainability parameters. Conclusion: BEDCA is the only BDCA in Spain, built under European quality standards, proposed by EuroFIR. Conflict of interests: Interest conflict does not exist.

30

\section{Evolution of the habits in alimentation and physical activity of schoolchildren in Soria from 1999 to 2019}

C. Julián Adell ${ }^{a}$, D. Garrido Gonzalo ${ }^{a}$, JA. Rodriguez Diez ${ }^{a}$, R. Andrés Alcoceba ${ }^{a}$

${ }^{a}$ Campus Duques de Soria. Universidad de Valladolid. Soria, España.

Background: Childhood obesity is one of the most severe public health problems of the 21th century. It's estimated that more that 41 million children around the world are overweight or obese. Objectives: To know the habits of physical activity and alimentation in a sample of school children who attend the canteen of public schools in the capital of Soria and analyze their relationship with adherence to the Mediterranean diet. Methods: Accidental sample of school children between the ages of 5 and 15 who are enrolled during the 2018-19 school year in 4 public schools in Soria that eat at school and voluntarily accept to participate in the study. All participants have completed the KIDMED (Med Clin (Barc) 2003; 121 (19): 725-32) and physical activity (Ref) questionnaire. The KIDMED questionnaire analyzes the compliance with the Mediterranean diet by means of 16 questions. Test score from 0 to 12 and allows to classify the quality in three different groups: $\leq 3$ poor quality, 4-7 average quality and $\geq 8$ top-quality. To obtain the profile of the diet, the food record survey for 3 days (one holiday) has been used. The food intake has been transformed into macronutrient intake through the NutrIber v1.1.5 program. The physical activity questionnaire allows to classify the respondents in sedentary, with moderate activity/intense/very intense. Results: The 
preliminary results allowed us to observe that the adherence to the MD in school canteens in Soria can be better than previous decades with an average value of the KIDMED index of 7.73 also we observed that there is a positive correlation between adherence to the Mediterranean diet and degree of activity. Conclusion: The adherence to the MD in the school canteens of Soria is better than in previous decades. Conflict of interests: There is no conflict of interest that could compromise the impartiality and independence of this work.

31

\title{
Is the gut-metabolite urolithin A responsible for neuroprotective properties of pomegranate extracts?
}

\author{
G. Cásedas ${ }^{a}, F \cdot$ Les $^{a}$ V. López ${ }^{a}$
}

${ }^{a}$ Department of Pharmacy, Faculty of Health Sciences, Universidad San Jorge, Villanueva de Gállego, Zaragoza, Spain.

Background: Urolithin A is the major gut metabolite of elagitannins and ellagic acid, which are some of the main polyphenolic compounds found in pomegranate extracts and products. In recent years, numerous studies have corroborated the beneficial effects of urolithin A and their possible protective effects on diabetes and metabolic syndrome1. One major cause of these disorders is oxidative stress which is also involved in neurodegenerative pathologies. At this point, we hypothesize wheter urolithin A could also be of neurotherapeutic interest to prevent or treat certain disorders. Objectives: To evaluate the effects of urolithin $\mathrm{A}$ on in vitro pharmacological models in relation with neurodegenerative diseases. In vitro antioxidant activity, enzyme inhibition bioassays and neuronal cultures were performed in order to support the potential bioactivities of urolithin A. Methods: For this purpose, enzyme inhibition bioassays of tyrosinase, acetylcholinesterase, and monoaminoxidase A, all involved in neurodegeneration, were carried out in cell-free systems. Likewise, DPPH, ORAC and superoxide radical in vitro scavenging activities were measured. Finally, neuroblast (Neuro-2a) cells derived from mice were subjected to oxidative stress and the effects of the metabolite were evaluated. Results: Urolithin A dose-dependently inhibited the enzymes tyrosinase, acetylcholinesterase and monoaminoxidase A. Furthermore, the metabolite displayed high antioxidant activity while oxidative stress results in the Neuro-2a cells are still being performed. Conclusion: These results might explain from an in vitro perspective the potential applications of urolithin A in the prevention of neurodegenerative-associated disorders. Conflict of interests: The authors declare no conflicts of interest.

32

\section{Ketogenic diet and physical activity intervention to weight loss analyzing different FTO and AdipoQ polimorfisms}

\author{
E. Moraleda ${ }^{a}$, I. Martínez-Argudo ${ }^{b}$, I. Rodríguez-Gómez ${ }^{c}$, I. Ara ${ }^{d}$, S. Aznar
}

${ }^{a}$ Grupo de Investigación PAFS. Universidad de Castilla la Mancha. Toledo, España.

'Área de Genética. Universidad de Castilla la Mancha. Toledo, España.

'Grupo de Investigación GENUD.Universidad de Castilla la Mancha. Toledo, España.

${ }^{\mathrm{d}}$ Grupo de Investigación GENUD. Universidad de Castilla la Mancha. Toledo, España.

${ }^{\mathrm{e}}$ Grupo de Investigación PAFS. Universidad de Castilla la Mancha. Toledo, España.

Background: Obesity is a global epidemic that raises cardiovascular and metabolic problems. Many studies have shown that ketogenic diets (KD) are capable of inducing improvements in cardiovascular risk parameters. However, there's paucity of research in KD together with physical activity (PA). Objective: To ensure that this study wasn't biased by the genetic characteristics of the sample, the genetic analyzes of the AdipoQ and FTO genes were carried out at baseline, since these genes are associated with an increased risk of obesity.The aim was to evaluate the effectiveness of KD in changing body composition in a sample of 8 of overweight subjects who also undergo either a continuous or interval PA protocol. Methods: The sample was randomly assigned into two groups: 4 subjects with intermittent training (IT) vs 4 subjects with continuous training (CT). All of them undertook a KD for 4 weeks (baseline mean fat mass of subjects with IT was $24.05 \mathrm{Kg} \pm 5.2$ and $\mathrm{CT}$ subjects had a mean fat mass of $24.34 \mathrm{Kg} \pm 5.1$ ). Body composition parameters 
were measured using DXA scan pre and post intervention. Genotype of FTO and AdipoQ genes polimorfisms were measured to assess possible bias. Results: There was a significant improvement $(p<0.05)$ in weight loss $(-4.6 \mathrm{Kg})$ in CT group and $(-3.45 \mathrm{Kg})$ in IT group; and in the amount of body fat mass loss throughout the sample $(-3.4 \mathrm{Kg}$ in CT group vs $-2.5 \mathrm{Kg}$ in IT group). As to FTO gene study, all of IT subjects had TT Genotype but CT subjects showed variety of genotypes. In regards to AdipoQ, in IT subjects all had GG genotype, but within CT subjects showed different genotypes. Conclusion: In a convenience sample of 8 subjects it has been shown that a KD contributed in weight loss and fat loss significantly, either with IT or CT. The results of this study appear to have been unaffected by genetic makeup. Conflict of interests: The authors declare no conflicts of interest.

\title{
Assessment of beverage intake and its relation with fitness level in adolescents
}

\author{
R. Ramírez-Domínguez ${ }^{a}$, Rodríguez-Díez $^{a}$ \\ ${ }^{\mathrm{a}}$ Department of Public health, Campus Duques de Soria. Facultad de Fisioterapia. Universidad de \\ Valladolid, Spain.
}

Background: Water is the main constituent of the human body and therefore its adequate intake is essential to maintain a state of euhydration which facilitates an optimal level of health. Hydration is formed as a habit of daily life necessary and educable. Moreover, adolescents are an especially sensitive population to acquire healthy habits. At the same time, there is scientific evidence which indicates that an optimal state of hydration is related with an optimal performance. From here, it is intended to evaluate the intake of beverages by teenage and establish their relationship with the level of physical condition measured by V02max. Methods: 28 men an 51 women students of the CIP ETI Tudela and the Faculty of Nursery of Campus Duques de Soria age of $18.87 \pm 1.7$ years were measured to evaluate the intake of beverages through the questionnaire from the PREDIMED PLUS study (Adapted from Hendricks et al) and on the other hand, the level of physical condition with the Forest Service step Test, obtaining the value of V02max through indirect estimation and with the use of heart rate monitors. The statistical treatment of the data will be carried out with the statistical package SPSS 22.0 using as statistical significance value $(p<0.05)$. Results: Results show no significant in total beverage intake differences between men $(2796.42 \pm 968.01 \mathrm{~mL})$ and women $(2499.69 \pm 1080.19 \mathrm{~mL}) \mathrm{p}=0.085$. Physical fitness measured by V02max correlated with bottled water intake $(\mathrm{p}=0.014)$ and with sports drinks $(\mathrm{p}=0.000)$. Conclusion: Beverage intake of CIP ETI Tudela and the Faculty of Nursery of Campus Duques de Soria adolescents students are susceptible of improvement. Moreover, suggest that a higher physical fitness level may be influenced by a conscious beverage intake. Conflict of interests: The authors declare no conflicts of interest.

\section{4}

\section{The levels of plasma leptin in relation to hormonal changes during the menstrual cycle in elite women volleyball players}

\author{
E. Marquez Calle ${ }^{a}$, C. Navas Ferrer ${ }^{a}$, B. Samper Lamenca ${ }^{a}$, C. Moro Lagares ${ }^{a}$ \\ ${ }^{a}$ Campus Duques de Soria. Universidad de Valladolid, Soria, Spain.
}

Background: The aim of this study is to assess the plasma leptin in relation to hormonal changes during three menstrual cycles in 16 elite women volleyball players during tournament. Methods: The study involved 16 healthy, normal-weight women. Anthropometric parameters, body composition and levels of plasma leptin in adition to serum FSH, TSH LH, estradiol, prolactine and progesterone concentrations were measured during a morning fasting state six times, between days 8 and 10 of the follicular phase and between 21 and 23 of the luteal phase of each subject in three menstrual cycles. Results: Our results reveled variable levels of leptin and this effect was significantly associated with changes in the secretion of several sex hormones. Conflict of interests: The authors declare no conflicts of interest. 


\title{
University consumers' perception of calcium health claims from dairy products. Preliminary results
}

\author{
P. Romero Marco ${ }^{a}$, Y. Larriva González ${ }^{a}$ \\ ${ }^{a}$ Campus Duques de Soria. Universidad de Valladolid, Soria, Spain.
}

Background: The purpose of this work is to present the preliminary results of purchase intent of dairy products with calcium health claims among university consumers. Dairy products (e.g. milk, yoghourt or cheese) of different brands usually bring particular calcium health claims to inform consumer about specific health benefits (e.g. stronger bones). Consumer purchase intent of these products may be influenced by marketing strategies, their perception of healthiness, gender, position, nationality or age among others. Methods: To do so, a pilot internet-based survey was conducted for a group of Spanish university consumers. Then, a statistical conjoint-type analyses was performed to describe to which extend dairy product, brand and calcium health claims influence in Spanish university consumers according to several socio-demographic factors. Conclusion: Once the questionnaire and preliminary results are fully validated a large-scale cross-international internet based survey will be launched. Conflict of interests: The authors declare no conflicts of interest.

Pectin's control of intestinal microbiota prevents liver injury in a mouse model of NASH

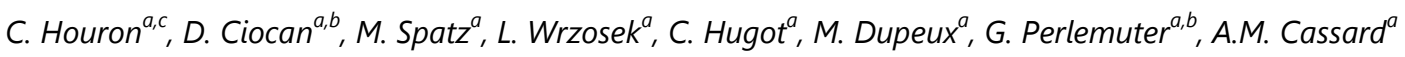

aINSERM U996, Inflammation Chemokines and Immunopathology, Faculté de Médecine-Univ Paris-Sud, Université Paris-Saclay, Clamart, France.

${ }^{\mathrm{b}} \mathrm{AP}-\mathrm{HP}$, Hepatogastroenterology and Nutrition, Hôpital Antoine-Béclère, Clamart, France

'Institut de recherche SERVIER, Suresnes, France.

Background: Non-alcoholic steatohepatitis (NASH) is a chronic liver disease with an unceasing prevalence increase, in line with the global obesity epidemic. If genetic and environmental components are linked to disease progression, it have been proved that intestinal microbiota plays a causal role in NASH pathogenesis. The various methods enabling control of intestinal microbiota, pre-, probiotics and faecal transplant still require adjustments to improve NASH management. Objective: With this in mind, it remains necessary to understand the mechanisms through which intestinal microbiota works. We have assessed the protective effect of pectin on liver damage in a mouse model fed with a high-fat diet (HFD) and we have correlated it with intestinal microbiota changes and gut damage. Methods: Five week-old C57BL6 male mice were fed with normal diet (ND) or high-fat diet (HFD) with or without pectine $(0.4 \%$ or $2 \%)$ for four months. During the experimental periods, the animals were maintained at $23^{\circ} \mathrm{C}$, with $12 \mathrm{~h}$ light/dark cycles and fed ad libitum with free access to water. At the end of the experimental period, liver damage was evaluated through determination of plasma levels of ALT, hepatic triglycerides dosage, histological features and mRNA expression of specific cytokines and chemokines. Liver damage were correlated to intestinal microbiota, analyzed by high-throughput sequencing targeting the 16s ribosomal DNA V3-V4 region and with intestinal barrier alterations assessed through histological analyses and qPCR. Results: Mice fed with HFD show steatosis, increased ALT and increased pro-inflammatory markers. These effects are prevented with pectin supplementation in a dose-dependent manner. Weight gain associated with HFD is classically associated with impairment of glucose tolerance. Assessment of glucose tolerance highlights the fact that HFD mice have decreased glucose tolerance when compared to ND mice, regardless of pectin supplementation. Mice fed with HFD + pectin $2 \%$ have an increased calorie intake compared to other groups of HFD mice, despite a similar weight gain. Histological analyses of brown adipose tissue of these mice reveal an increased activation process that may explain physiological differences between the groups. Global analysis of intestinal microbiota reveal that pectin influences intestinal microbiota in a dose-dependent manner, in particular by reversing the Firmicutes/Bacteroidetes ratio. In HFD mice, the gut epithelium is impaired and pectin supplementation seems to be able to prevent this damage by stimulating mucus and anti-bacterial peptides production leading to gut barrier restoration. Conclusion: Preliminary results highlight that pectin 
prevents the onset of liver injury in a mice model of NASH, notably through changes in intestinal microbiota and improvement of intestinal barrier. Faecal transplants have been performed using intestinal microbiota modified by pectin in order to determine of the beneficial effects of pectin on liver injury and gut barrier are only due to changes in intestinal microbiota. Conflict of interests: The authors declare no conflicts of interest.

Antioxidant and cardio-protective effects of EPA in early low-severity sepsis through UCP3- and SIRT3-upholding of the mitochondrial redox potential

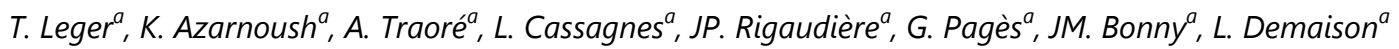 \\ ${ }^{a}$ Université Clermont Auvergne, INRA, UNH, Unité de Nutrition Humaine, CRNH, Auvergne, Clermont- \\ Ferrand, France.
}

Background: Sepsis still causes death, often through cardiac failure and mitochondrial dysfunction. Dietary $\omega 3$ polyunsaturated fatty acids are known to protect against cardiac dysfunction and sepsis lethality. Objectives: This study set out to determine whether early low-severity sepsis alters the cardiac mitochondrial function in animals fed a Western-type diet, and whether dietary eicosapentaenoic acid (EPA) administration protects the myocardium against the deleterious effects of sepsis, and if so to seek possible mechanisms for its effects. Methods: Rats were divided into two groups fed either a $\omega 3$ PUFA-deficient diet ("Western diet", DEF group) or an EPA-enriched diet (EPA group) for 5 weeks. Each group was subdivided into two subgroups: sham-operated rats and rats subjected to cecal ligation and puncture (CLP). In vivo cardiac mechanical function was examined, and mitochondria were harvested to determine their functional activity. Oxidative stress was evaluated together with several factors involved in the regulation of reactive oxygen species metabolism. Results: Sepsis had little effect on cardiac mechanical function, but strongly depressed mitochondrial function in the DEF group. Conversely, dietary EPA greatly protected the mitochondria through a decreased oxidative stress of the mitochondrial matrix. This last was probably due to an increased uncoupling protein-3 expression, already seen in the sham-operated animals. CLP rats in the EPA group also displayed increased mitochondrial sirtuin-3 protein expression that could reinforce the upholding of oxidative phosphorylation. Conclusion: Dietary EPA preconditioned the heart against septic damage through several modifications that protect mitochondrial integrity. This preconditioning can explain the cardio-protective effect of dietary EPA during sepsis. Conflict of interests: The authors declare no conflicts of interest.

38

\title{
Physical activity in modelling children's body composition
}

\author{
I. Iglesia Altaba ${ }^{a}$, P. Ferrer-Santos ${ }^{b}$, M.L. Miguel-Berges ${ }^{b}$, P. Flores-Barrantes ${ }^{b}$, P. Samper-Villagrasa ${ }^{c}$, G. Martínez- \\ Rodríguez ${ }^{c}$
}

anstituto de Investigación Sanitaria de Aragón, España.

${ }^{b}$ Growth, Exercise, Nutrition and Development (GENUD) Research Group, Universidad de Zaragoza, España.

'Department of Pediatrics, Universidad de Zaragoza, España.

Background: Physical activity may have an impact in children's body composition. Objectives: To elucidate the association of moderate-vigorous physical activity (MVPA) and body composition of children from Aragón accounting for socioeconomic, dietary and sedentary behavior effects. Methods: The longitudinal cohort 'Growth and Feeding during Lactation and Early Childhood in Children of Aragon (CALINA in Spanish)' included 1,629 born children from Aragon (Spain) in 2009. From those, after 7 years, 339 children (176 boys and 163 girls) were assessed. Body composition (using Dual X-ray absorciometry DXA-), diet quality index (DQI) (using a food frequency questionnaire-FFQ-), sedentary behaviours by questionnaire, and physical activity (using accelerometry-actigraph 3GTX-) were evaluated, as well as if they were or not rapid weight gainers during the first year of life, were also considered. Results: The longitudinal cohort 'Growth and Feeding during Lactation and Early Childhood in Children of Aragon (CALINA in Spanish)' included 1,629 born children from Aragon (Spain) in 2009. From those, after 7 years, 339 children (176 boys and 163 girls) were assessed. Body composition (using Dual X-ray absorciometry -DXA-), diet 
quality index (DQI) (using a food frequency questionnaire-FFQ-), sedentary behaviours by questionnaire, and physical activity (using accelerometry-actigraph 3GTX-) were evaluated, as well as if they were or not rapid weight gainers during the first year of life, were also considered. Conclusion: Still there is an important physical activity gap between boys and girls. In boys, physical activity is associated with both lean and fat mass while only with fat mass in girls. More research is required to elucidated the effects on health in children both of PA and body composition. Conflict of interests: None.

\title{
Healthy eating in Preschools and Elementary Schools: The SI! Program
}

\author{
P. Bodega ${ }^{a}$, G. Santos-Beneit ${ }^{a}$, C. Rodríguez ${ }^{a}$, X. Órrit $^{a}$, C. Carral ${ }^{a}$, D. Haro ${ }^{a}$, A. de Cos-Gandoy ${ }^{a}$, M. Miguel $^{a}$ \\ ${ }^{a}$ Equipo científico. Fundación SHE. Barcelona. España.
}

Background: The SHE Foundation has developed the SI! Program: an intervention to promote cardiovascular (CV) health in children through four components (diet, physical activity, human body and heart, and emotions). Objective: To describe the diet component of the SI! Program in Preschools (3-6 years) and Elementary Schools (6-12 years). Methods: The intervention includes 10 hours/year about healthy diet, providing tools to acquire healthy dietary habits through the knowledge of foods properties. In Preschools the intervention is based on playing, while in Elementary school the activities are based on experimentation. In both cases, most of the intervention is implemented in the classroom led by the teachers. An additional home activity is included to reinforce with the family the concepts learned in class. Results: The intervention in Preschools is based on promoting fruits and vegetables intake using healthy recipes and encouraging children to eat a variety of healthy foods (different colors). Moreover, they learn the importance of breakfast; the recommended servings from each food group and appropriate serving size. In Elementary schools the intervention focuses on reinforcing the concepts learnt in Preschool. Children also learn the relationship between salt, sugar and different types of fat and CV health. In both stages, the intervention aims for children to care for their CV health. Both programs are currently being implemented in 214 schools in Spain. All stages of the SI! Program are assessed with a randomized controlled trial currently ongoing (20142020). Conclusions: The promotion of heathy diet during childhood could be a great tool to prevent CV disease. It is essential to encourage the intake of fruit and vegetables and to adapt the intervention to age. The SI! Program for Preschoolers has shown to be effective to improve knowledge, attitudes and habits related to CV health and we hope to find similar results in Elementary Schools. Conflict of interests: The authors declare no conflicts of interest.

40

\section{Protective effect of hepatic fibroblast growth factor 21 (FGF21) on hepatic steatosis and liver damage}

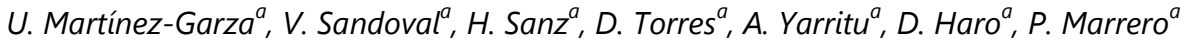

aDepartment of Nutrition, Food Science and Gastronomy, Faculty of Pharmacy and Food Science, Torribera Campus. University of Barcelona. Barcelona, Spain.

Background: Non-alcoholic fatty liver disease (NAFLD) is a multi-step chronic liver disease essentially characterized by an accumulation of lipids in the liver. It is strongly associated to obesity and metabolic disorders and goes from steatosis to various fibrosis states. The last stages of NAFLD cause serious damages in the liver and hepatic dysfunctions. It has been reported that FGF21 is capable to reduce the fat content in the liver and to decrease hepatic fibrosis and inflammation. Objective: The objective of this study was to analyze the effects of hepatic FGF21 on liver steatosis caused by tunicamycin administration. Tunicamycin is an inducer of the endoplasmic reticulum (ER) stress and an inhibitor of hepatic gluconeogenesis inhibitor, which causes an acute liver steatosis. Methods: Male mice C57BL6/J of 10 weeks of age with specific hepatic depletion of FGF21 (LFKO) and their respective littermates (LoxP) were subjected to tunicamycin administration $(1 \mathrm{mg} / \mathrm{kg}$ ) or vehicle (PBS) for $24 \mathrm{~h}$ prior to the liver extraction. Gene expression analysis was carried out by qPCR and protein activity by ELISA. The main genes involved in the lipid homeostasis and liver function were analyzed. Results: In the liver, tunicamycin causes an accumulation of triglycerides and 
an increase of FGF21 expression. Moreover, hepatic FGF21 induces the expression of immunoglobulin heavy chain-binding protein (BiP) and the C/EBP homologous protein (CHOP). These two proteins are both implied in the Unfolded Protein Response (UPR). The UPR is an adaptive cell response to excessive lipid accumulation and ER stress in liver. Also, hepatic FGF21 improves liver damage markers in serum, lowering circulating transaminases (ALT and AST). Conclusion: Altogether shown that hepatic FGF21 could prevent liver damage caused by tunicamycin by inducing the UPR, thus reducing the ER stress and finally improving the liver functionality. Conflict of interests: The authors declare no conflicts of interest.

41

\section{Effect of Moringa oleifera dry leaf powder on postprandial glucose and insulin response}

Nova Rebato, E. ${ }^{a}$, Gómez Martínez,S. ${ }^{a}$ Dueñas Martín ,M. ${ }^{a}$, Díaz Prieto,Le ${ }^{a}$, Vicente Castro,l. ${ }^{a}$, Picón César,MJ. ${ }^{b}$, Godino García,M. ${ }^{c}$, Marcos Sánchez,A. ${ }^{a}$

${ }^{a}$ Instituto de Ciencia y Tecnología de Alimentos y Nutrición (ICTAN)-CSIC, Madrid, España

bervicio de Endocrinología y Nutrición, Hospital Virgen de la Victoria, Málaga, España

'E.T.S. de Ingeniería de Montes, Forestal y del Medio Natural, Universidad Politécnica de Madrid, Madrid, España

Moringa oleifera (MO) is a plant rich in nutrients and phytochemicals, cultivated in tropical and subtropical areas and already introduced also in the Iberian Peninsula. Multiple bioactivities of plant extracts have been observed in in vitro assays and animal experiments have revealed a variety of medicinal purposes, including hypoglycemic activity. However, human studies are still very scarce.

This is a pilot study on the effect of different doses of MO of peninsular origin on postprandial glucose level in non-diabetic subjects.

Five adult subjects were included ( 2 with normal fasting glucose and 3 with pre-diabetic fasting glucose history). A standard breakfast, plus, respectively, one placebo capsule (PLC), or 1, 3 and 6 capsules containing 400mg of MO dry leaf powder (1-, 3- and 6-MO, respectively) were administered on 4 consecutive visits, one week apart. The basal and postprandial $(30,60,90,120,150$ and $180 \mathrm{~min})$ glucose and insulin values were measured. The areas under the curves (AUC) were calculated and compared for MO treatments vs. placebo (Wilcoxon test). Statistical significance was set at $\mathrm{p}<0.05$.

Differences were found between the AUC for glucose with 1-MO compared to PLC. No significant differences were found with 3-MO and 6-MO compared to PLC, probably because of the high variability among subjects in fasting glucose and postprandial response. However, mean postprandial glucose levels at all time points were lower with all MO doses compared to PLC. No differences were found either between AUC for Insulin with any of the 3 MO doses compared to PLC; however, Insulin peaks were higher and earlier with all MO doses vs. PLC.

This pilot study suggests that potentially beneficial postprandial effects of MO dry leaves in glucose control could be found in a study with higher sample size and with a more similar glucose metabolism among participants. Conflict of interests: There is no conflict of interest to declare.

42

\section{Consensus on sustainable palm oil and human health}

Gesteiro, E. ${ }^{a}$, Guijarro,L. , Sánchez-Muniz,FJ. ${ }^{c}$, Vidal-Carou,MC. ${ }^{d}$, Troncoso,A. ${ }^{e}$, Venanci,Ll. ${ }^{f}$ Jimeno,.$^{g}$, Quilez,J. ${ }^{h}$, Anadón, $A^{i}$, González-Gross, $M^{a}$

${ }^{a}$ ImFine Research Group. Departamento de Salud y Rendimiento Humano. Facultad de Ciencias de la Actividad Física y del Deporte-INEF. Universidad Politécnica de Madrid. Madrid, Spain.

${ }^{b}$ Departamento de Periodismo II. Facultad de Ciencias de la Información. Universidad Complutense de Madrid.

'Departamento de Nutrición y Ciencia de los Alimentos. Facultad de Farmacia. Universidad Complutense de Madrid. Madrid, Spain 
${ }^{\text {d} D e p a r t a m e n t ~ d e ~ N u t r i c i o ́, ~ C i e ̀ n c i e s ~ d e ~ I ' A l i m e n t a c i o ́ ~ i ~ G a s t r o n o m i a . ~ C a m p u s ~ d e ~ l ' A l i m e n t a c i o ́ n ~ d e ~}$ Torribera. Facultat de Farmàcia i Ciències de I'Alimentació. Universitat de Barcelona. Barcelona, Spain

eDepartamento de Nutrición y Bromatología, Toxicología y Medicina Legal. Universidad de Sevilla. Sevilla, Spain

${ }^{f}$ Asesor independiente

${ }^{9}$ Departamento de Producción Agraria. ETSI Agronómica, de Alimentación y Biosistemas. Universidad Politécnica de Madrid. Madrid, Spain

hDirector de Tecnología en Europastry. S.A.

'Departamento de Farmacología y Toxicología. Facultad de Veterinaria. Universidad Complutense de Madrid. Madrid, Spain.

Introduction. There is a great concern in the Spanish population regarding palm oil (PO) and its effects on health, non-communicable diseases (NCD), toxic contaminants, and deforestation among others.

Aims. To establish a consensus positioning about PO based on current scientific state of the art.

Methods. Ten experts attended a meeting for discussing about critical aspects of PO.

Results. The main issues were I) Sustainability: a) The roundtable for sustainable PO (RSPO) was created in 2004 making palm one of the sustainable crop leaders. b) The implementation of $100 \%$ sustainable PO in 2020 for Europe is a goal of the Amsterdam Declaration. c) Raising awareness on choosing sustainable products to improve environment and economy in producing countries is a priority need. II) Nutrition: a) The SFA/MUFA/PUFA ratio in PO is 4.7/3.8/1.0. The whole diet rather than an isolated food should be evaluated. b) Recent studies are re-evaluating the role of SFA in cardiovascular risk. c) There is no evidence supporting changes in the fat intake recommendations (20-35\%E, AGS>10\%E). III) Food industry. a) The use of certified sustainable PO is gradually increasing IV) Food safety. a) There is no evidence associating PO and human cancer incidence. b) JECFA and EFSA have established the Tolerable Daily Intake (TDI) for the toxic contaminants 3-monocloropropanodiol esters (3-MCPDE) and glycidil esters (GE). Consequently, the European Commission has modified its contaminant regulation for GE.

Conclusions: Palm is a high efficiency crop considering oil litres/Ha, and can be a good resource in order to feed a growing world population $>7500 \mathrm{M}$ people. PO is used in western countries as an ingredient of processed foods, whose intake is recommended to be sparing. There is a lack of studies analysing PO intake in Europe. Investigation, technology and communication should collaborate to assure an objective information flow. Conflict of interests: None. 


\title{
The food contaminant semicarbazide reduces body weight gain and adiposity in mice in a manner not reproduced by other amine-oxidase inhibitors
}

\author{
C. Carpéné ${ }^{a}$, N. Boulet ${ }^{a}$, A. Lasbories ${ }^{a}$, A. Bouloumie ${ }^{a}$, A. Briot ${ }^{a}$
}

${ }^{a}$ Eq1, INSERM U1048, Institut des Maladies Métaboliques et Cardiovasculaires \& I2MC, Université Paul Sabatier, Toulouse, France.

Background: Traces of semicarbazide have been found in food at doses ranging from 0.09 to $1.27 \mu \mathrm{g} / \mathrm{kg}$ (1) i.e. well below the safe levels estimated for this toxic food contaminant, the no-observed-adverse-effect-level of which ranges between 0.6 and $3.9 \mathrm{mg} / \mathrm{kg} /$ day in rats. However, semicarbazide is a marker of nitrofuran abuse, being a metabolite of the compound azodicarbonamide, used as a bleaching additive for flour treatment, or as a blowing agent for packaged foods including babyfoods (2). Semicarbazide is also a byproduct of the veterinary antibiotics nitrofurazone and furaltadone (3) and is considered as an endocrine disrupter (4). Objectives: Since it has been described that oral semicarbazide treatment limits fat accumulation in rodents at oral dose of $1000 \mathrm{ppm}$, or reaching $1.3 \mathrm{mmol} / \mathrm{kg}$ body weight $(\mathrm{bw})(5,6)$, and considering that semicarbazide inhibits copper-containing amine oxidases (CAO), abundant in adipose tissue and liver, it was investigated how other CAO inhibitors, especially of novel-generation, could limit adipose tissue expansion. Methods: BTT-2052, phenelzine, and pentamidine have been administered orally in the drinking water of $\mathrm{FVB} / \mathrm{n}$ male mice at doses one hundred or ten times lower than that of semicarbazide, in agreement with their higher capacity to inhibit CAO: 14, 136 and 136 vs $1360 \mu$ moles/kg bw/d for 8 weeks, respectively. Results: BTT 2052 and phenelzine - but not pentamidine - treatments did not notably alter food and water consumption during the 8-week oral treatment. The two former agents inhibited CAO activity in adipose deposits of mice, therefore reproducing the inhibitory action of semicarbazide. Pentamidine did not inhibit amine oxidase activity. However, no treatment reduced significantly weight gain and fat accumulation, indicating that $\mathrm{CAO}$ inhibition was not sufficient to limit fat deposition. Conclusion: Although our data suggest relationships between adipocyte CAO activity and energy storage, we propose that CAO inhibition is not the sole mechanism by which the food contaminant semicarbazide exhibits antiobesity effects. Conflict of interests: Authors declare no conflicts of interest. References: (1) Radovnikovic et al. Residue analyses and exposure assessment of the Irish population to nitrofuran metabolites from different food commodities in 2009-2010.Food Addit Contam Pt A. 2013;30:1858-69. (2) Maranghi et al. Effects of the food contaminant semicarbazide following oral administration in juvenile Sprague-Dawley rats Food Chem Toxicol. 2009;47:472-9. (3) Pereira et al. Implications of the use of semicarbazide as a metabolic target of nitrofurazone contamination in coated products. Food Addit Contam. 2004;21:63-9. (4) Maranghi et al. The food contaminant semicarbazide acts as an endocrine disrupter: Evidence from an integrated in vivo/in vitro approach. Chem Biol Interact. 2010;183:40-8. (5) Takahashi et al. A ninety-day toxicity study of semicarbazide hydrochloride in Wistar Hannover GALAS rats. Food Chem Toxicol 2009, 47, 2490-8. (6) Mercader et al. Oral administration of semicarbazide limits weight gain together with inhibition of fat deposition and of primary amine oxidase activity in adipose tissue. J Obes 2011, 2011, 475786.

\section{Endothelial damage biomarkers and metabolic health status in prepubertal children born to mothers with pregnancy hypertension}

\author{
A.I. Ruperez ${ }^{a, b}$, G. Pérez-Gimeno ${ }^{a, b}$, C.M. Aguilera ${ }^{c, d}$, M. Gil-Campo ${ }^{e, f}$, E.M. González-Gil ${ }^{c, d}$, L.A. Moreno ${ }^{a, b}$, R. Leis ${ }^{g, h}$, \\ G. Bueno $0^{i j}$ \\ ${ }^{a}$ Departamento de Fisiatría y Enfermería, Universidad de Zaragoza. \\ ${ }^{b}$ Growth, Exercise, Nutrition and Development (GENUD), Edificio SAl. Zaragoza. Spain. \\ 'Departamento de Bioquímica y Biología Molecular II, Instituto de Nutrición y Tecnología de los Alimentos, \\ Universidad de Granada. \\ ${ }^{d}$ Centro de Investigación Biomédica. Granada. Spain. \\ eUnidad de Investigación en Pediatría y Metabolismo, Hospital Universitario Reina Sofía. \\ fInstituto Maimónides de Investigación Biomédica (IMBIC). Córdoba. Spain.
}


9Departamento de Pediatría, Unidad de Investigación en Nutrición, Crecimiento y Desarrollo de Galicia.

hInstituto de Investigación Sanitaria de Santiago de Compostela (IDIS), Universidad de Santiago de Compostela.

Santiago de Compostela, Spain.

'Departamento de Pediatría, Universidad de Zaragoza. Zaragoza, Spain.

jHospital Universitario Lozano Blesa. Zaragoza, Spain.

Background: Children born to mothers with pregnancy hypertension (PH) are at increased risk of obesity and metabolically unhealthy status (MU status). However, it is unclear whether PH is associated with altered circulating biomarkers of cardiovascular disease. Objectives: To examine the plasma biomarkers of endothelial damage and inflammation in children born to mothers who had PH. Methods: 112 prepubertal children (54 females; 78 with overweight/obesity) aged 6-12 years were recruited in three Spanish cities. The presence of $\mathrm{PH}$ and general perinatal information was recorded, along with later offspring anthropometric variables, blood pressure (BP) and plasma biomarkers of endothelial damage and inflammation. Children were classified according to their BP, triacylglicerides, HDL-C, glucose and HOMA-IR age-, sex- (for BP, triacylglicerides and HDL-C) and height-specific (for BP) percentile values. Linear regression, adjusted for age, sex, recruitment center and birthweight, was used to compare biomarker concentrations between children born to mothers who had PH or not. The associations shown by matrix metalloproteinase-9 (MMP-9) and active plasminogen activator inhibitor-1 (aPAI-1) with other biomarkers were studied by correlation analysis and multiple linear regression. Results: PH was associated with children's risk of having $<10$ th percentile age- and sex-specific HDL-C values (OR=6.73, 1.45-31.20, $\mathrm{P}=0.015$ ), higher offspring plasma MMP-9 (111.8 $\pm 78.8 \mu \mathrm{g} / \mathrm{L}$ in PH-offspring vs. 74.1 $\pm 34.32 \mu \mathrm{g} / \mathrm{L}$ in non-PH-offspring; $\mathrm{P}=0.003)$ and lower glucose and aPAI-1 concentrations $(5.6 \pm 3.5 \mu \mathrm{g} / \mathrm{L}$ in PH-offspring vs. $13.6 \pm 16.2 \mu \mathrm{g} / \mathrm{L}$ in non-PH-offspring; $P=0.027)$. Plasma MMP-9 was positively associated with resistin $(\beta=0.27, P=0.012)$, HGF $(\beta=0.25, P=0.023), P H(\beta=0.20, P=0.031)$ and soluble vascular cell adhesion molecule- $1(\beta=0.26, P=0.036)$, whereas a-PAI1 was positively associated with total PAI-1 $(\beta=0.65, P<0.001)$ and leptin $(\beta=0.26, P=0.043)$. Conclusion: Prepubertal offspring of mothers who had PH show increased circulating concentrations of MMP-9, which was associated with other endothelial damage biomarkers. The prevention and treatment of maternal PH is essential to avoid offspring endothelial damage.

\title{
Interaction between host microRNAs and gut microbiota in obese subjects
}

\author{
T. Silveira Assmann ${ }^{a}$, A. Cuevas-Sierra ${ }^{a}$, F.I. Milagro ${ }^{a}$, J.I. Riezu-Boj ${ }^{a}$, J.A. Martínez $^{a}$
}

${ }^{a}$ Department of Nutrition, Food Science and Physiology; Center for Nutrition Research, University of Navarra, Pamplona, Spain.

Background: Obesity is resulting in a global epidemic and an independent risk factor for several metabolic disorders. Although variation in gut microbiome composition has been linked with obesity, the factors that mediate the interactions between obesity and the microbiome are poorly understood. MicroRNAs (miRNAs) are small non-coding RNAs that regulate gene expression and they are known to be associated with obesity and metabolic diseases. In addition, recent studies suggest that host miRNAs may also regulate bacterial growth and influence the composition of the gut microbiome. However, the role of miRNAs in hostmicrobiome interactions is still unknown, especially in the obesity conditions. Objectives: To investigate the interactions between circulating miRNA expression and microbiome composition in patients with obesity compared to normal weight individuals. Methods: The sample comprised 78 subjects with obesity (BMI: $30-40 \mathrm{~kg} / \mathrm{m} 2$ ) and 25 individuals with normal weight (BMI equal or less than $25 \mathrm{~kg} / \mathrm{m} 2$ ). The levels of 96 miRNAs were quantified in plasma using miRCURY LNA miRNA Custom PCR Panels (Exiqon). Fecal bacterial DNA sequencing was performed following the Illumina $16 \mathrm{~S}$ protocol, which is based on the amplification of the V3-V4 variable regions of the 16S rRNA gene. All statistical analyses were performed using SPSS 20.0. Results: A total of 26 miRNAs as differentially expressed in plasma of subjects with obesity compared to normal weight individuals were featured. Additionally, the expression of 14 miRNAs (miR-107, miR-103a3p, miR-142-5p, miR-222-3p, miR-221-3p, miR-183-5p, miR-183-5p, miR-130b-3p, miR-15a-5p, miR-33a5p, miR-210-3p, miR-144-3p, miR-185-5p, miR-130a-3p, and miR-21-5p) was significantly associated with the relative abundance of 4 bacterial species (Dorea longicatena, Banesiela intestinihominis, Bacteroides eggerthii, and Haemophillus parainfluenzae). miRNAs that correlated with obesity-associated bacteria regulate target genes that are relevant for energetic metabolism. Conclusion: This research characterized 
differential relationships between microbial community composition and miRNA expression in plasma of patients with obesity compared to normal weight individuals. Conflict of interests: No conflicts of interest.

\section{Histamine-degrading capacity of lyophilised legume sprouts}

O. Comas-Basté ${ }^{a}$ J. Rabell-González ${ }^{a}$, F. Pederzania , S. Sánchez-Pérez ${ }^{a}$, N.C. Muñoz-Esparza ${ }^{a}$, M.L. LatorreMoratalla ${ }^{a}$, M.T. Veciana-Nogués ${ }^{a}$, M.C. Vidal-Carou ${ }^{a}$

aDepartament de Nutrició, Ciències de I'Alimentació i Gastronomia. Facultat de Farmàcia i Ciències de I'Alimentació. INSA.UB. XaRTA. Universitat de Barcelona. Santa Coloma de Gramanet. Spain.

Background: Diamine oxidase (DAO), originally called histaminase, is the enzyme that performs the oxidative deamination of the primary amino group of histamine. In humans, intestinal DAO acts as a protective barrier towards exogenous histamine, specially from food. A deficiency of DAO may lead to excess the normal plasmatic levels of histamine and the subsequent appearance of the symptomatology of histamine intolerance. Certain legume sprouts have been described as a source of DAO, and thus could be a suitable component for the formulation of DAO supplements as a complementary treatment strategy for histamine intolerance. Objectives: To perform a screening of the in vitro histamine-degrading capacity of all commercial legumes and to study the stability of the enzymatic activity of the lyophilised product at different storage conditions. Methods: Lyophilised legume sprouts of 10 different species were obtained in our laboratory through the germination of the seeds and the subsequent freeze-drying. In vitro DAO activity was measured through an enzymatic assay coupled to UHPLC-FL analysis of remaining histamine. Results: Except for beans and mung beans, all analysed legume sprouts showed in vitro histamine-degrading capacity. Concretely, lyophilized pea and grass pea sprouts showed the greatest DAO activity $(0.40 \mathrm{mU} / \mathrm{mg})$. Freezing storage $\left(-20^{\circ} \mathrm{C}\right)$ of the lyophilized legume sprouts kept the enzymatic activity intact for at least 6 months. On the contrary, the storage in refrigerator $\left(2^{\circ} \mathrm{C}\right)$ or at room temperature $\left(\cong 20^{\circ} \mathrm{C}\right)$ supposed a marked decrease in the enzymatic capacity of the product. Conclusion: Lyophilised sprouts of certain legumes have been demonstrated to be a plant-origin source of DAO enzyme. This matrix could be a suitable component for the formulation of DAO enzymatic supplements to enhance histamine degradation at intestinal level. Further studies are needed to select the optimal formulation of the final product to guarantee the stability of its enzymatic capacity. Conflict of interests: Authors declare no conflicts of interest.

\section{Optimization of a non-invasive method of trancriptome analysis of human intestinal cells using fecal samples}

M. Sánchez-Campillo ${ }^{a}$, M.T. Pastor-Fajardo ${ }^{b}$, M. Sabater-Molina ${ }^{b}$, M.J. López-Andreo ${ }^{c}$, E. Morales $^{b}$, E. Larqué $^{a}$

aDepartment of Physiology, Faculty of Biology, University of Murcia, Spain.

${ }^{\mathrm{b}}$ Biomedical Research Institute of Murcia (IMIB-Arrixaca), Murcia, Spain.

'Biology Molecular Section (ACTI). University of Murcia, Spain.

Background: Gastrointestinal tract functionality is influenced by different factors which could affect the transcriptome of gut. Gut exfoliated cell gene expression from stool is closely matched with the transcriptome of the small gut mucosa. Since eukaryotic mRNA presents poly A sequence that distinguishes them from the prokaryotes transcriptome, we tested several methods to get a suitable one to conduct exfoliome analyses. Objectives: We aimed to optimize a non-invasive method for exfoliome analyses from feces. Methods: Stool sample of an adult was collected in RNALater®. Total RNA belonging to both intestinal and microbiota cells was purified from stool, and their integrity analyzed by Bioanalyzer. We treated RNA samples under different methods: using total isolated RNA (method 1); selective amplification using oligo dT in order to get a complementary cRNA from poly(A) RNA (method 2); using resin spin columns for binding poly A+ mRNA for washing and eluting bound eukariotic mRNA (method 3); method 3 plus method 2 (method 4). We performed RT-PCR and analyzed by qPCRthe expression of both GADPH (eukaryotic gene) and ribosomal 16S from Enterobacteriaceae (prokaryotic gene). Results: The method 1 showed the highest expression by $\mathrm{qPCR}$ of $16 \mathrm{~S}(\mathrm{Ct}=11.8 \pm 0.80)$ while the method 4 the lowest 
$(\mathrm{Ct}=28.27 \pm 0.04)$. The expression of GADPH was the highest in the method $2(\mathrm{Ct}=21.9 \pm 0.45)$ while the lowest using method $1(\mathrm{Ct}=29.78 \pm 0.17)$. The ratio of $\mathrm{Ct}$ from $\mathrm{GADPH} / 16 \mathrm{~S}$ to detect the highest eukaryote and lowest prokaryotic expression at the same time, showed method 4 as the most appropriate for subsequent microarray analyses (Ratio Ct GAPDH/16S; method 1=2.5, method 2=1.15, method 3=1.39, method 4=0.84).

Conclusion: The best method of purification eukaryotic mRNA for transcriptome analysis by microarray is isolation of total RNA from feces plus spin columns resin binding poly A+ followed by selective amplification using oligodT. We are evaluating the exfoliome by microarray in children under different growth pattern.

Conflict of interests: The authors declare no conflict of interests.

6

\title{
Dietary management of histamine intolerance: How do the presence of other amines influence on histamine degradation by the DAO enzyme?
}

\author{
S. Sánchez-Pérez ${ }^{a}$, F. Frongia ${ }^{a}$, O. Comas-Bastéa ${ }^{a}$ S. Hernández-Macías ${ }^{a}$, N.C. Muñoz-Esparza ${ }^{a}$, M.T. Veciana- \\ Nogués ${ }^{a}$, M.L. Latorre-Moratalla
}

aDepartamento de Nutrición, Ciencias de la Alimentación y Gastronomía, Facultad de Farmacia y Ciencias de la Alimentación, Universidad de Barcelona, España.

Background: Diamine-oxidase (DAO) is the main responsible for the metabolism of histamine and other diamines at the intestinal level. A DAO deficiency leads to an increase of histamine absorption from food and the appearance of symptoms associated with histamine intolerance (HI). Currently, the main strategy to avoid symptoms of $\mathrm{HI}$ is to follow-up a low-histamine diet, which excludes foods that patients relate to the onset of symptoms. The diets reported in the literature usually excluded foods rich in histamine but also other foods without or with very low levels of this amine. Objectives: To assess in vitro if the presence of other amines, such as putrescine or cadaverine, interfere on the degradation of histamine by DAO enzyme. Methods: The in vitro assay of DAO degradation was made with histamine alone and together with different concentrations of putrescine or cadaverine in equimolar proportion or at levels 4 times lower and higher. Histamine degradation was determined throughout 3 hours of enzymatic assay by UHPLC-FL. Results: Putrescine and cadaverine exerted an inhibitory effect on histamine degradation in vitro when were at equal or higher levels than that of histamine. Histamine was totally reduced at 90 minutes of the assay, whereas when concentrations 4 -fold higher of putrescine or cadaverine were added, the percentage of histamine degradation was reduced an $18 \%$ and $23 \%$, respectively. The presence of the other diamines delayed 30 minutes the total histamine degradation. Conclusion: The presence of putrescine and cadaverine reduces the degradation rate of histamine by DAO enzyme. Therefore, for the dietary management of HI, not only should be considered the foods with high levels of histamine but also those that could be an important source of these other amines, such as citrus, banana, sweet corn and green pepper. Conflict of interests: No conflict of interest.

\section{The Impact of Twelve Weeks of Tribulus Terrestris Supplementation on CrossFit ${ }^{\circledR}$ Performance and Hormonal Response in Recreational Athletes}

\author{
D. Fernández Lázaro ${ }^{a}$, J.F. Mielgo Ayuso ${ }^{b}$, A. Caballero García ${ }^{c}$, C.I. Fernández Lázaro ${ }^{d}$, A Córdova Martínez ${ }^{b}$
}

${ }^{a}$ Department of Cell Biology, Histology and Pharmacology, Faculty of Physiotherapy, University of Valladolid, Soria Campus, Soria, Spain.

${ }^{b}$ Department of Physiology, Faculty of Physiotherapy, University of Valladolid, Soria Campus, Soria, Spain. 'Department of Anatomy, Faculty of Physiotherapy, University of Valladolid, Campus of Soria, Soria, Spain.

${ }^{\mathrm{d}}$ Department of Preventive Medicine and Public Health, Faculty of Medicine, University of Navarra, Pamplona, Spain.

Background: Tribulus Terrestris extract (TTE) is an herbal nutritional supplement that appears to be a potent sport performance enhancer. CrossFit@ (CF) is a new but extremely popular method of exercise training that involves constantly varied functional movements performed at high intensity and strength. 
However, TTE information establishing its efficacy during sport practice is limited. Objectives: Determine the effects of TTE supplementation on CF performance on two common CF "Workouts of the Day" (WODs) and hormone response. Methods: Sixteen CF athlete's men (age: 38.4 \pm 3.8 years; body mass index 25.6 \pm 2.7 $\mathrm{Kg} / \mathrm{m} 2$ ) had at least 1 year of $\mathrm{CF}$ training experience were recruited from. Participants were randomized in a double-blind, randomized, crossover design. Athletes completed, on separate days, the WODs "Grace" (30 clean and jerks for time) and "CF Total" (1 repetition max: back squat, overhead press, and deadlift). Also, hormones as $\mathrm{T}$ and Cortisol (C) were assessed before and after 12 weeks of supplementation with TTE (750 mg) group treatment (GT) or a placebo (GC) immediately after training session. The percentage changes of the variables studied in each study group between the baseline (T1) and post-treatment (T2) tests were calculated as $\Delta(\%):[(\mathrm{T} 2-\mathrm{T} 1) / \mathrm{T} 1] \times 100$. Repeated-measures ANOVA with group factor were used to examine if the interaction of the different values was the same or different between phases $\mathrm{p}<0.05$. Results: Significant differences in favor of GT were observed in percentage changes of press (GC: $-8.52 \pm 5.54 \%$ vs. GT: $19 \pm 7.15 \% ; \mathrm{P}=0.044$ ) and T (GC $-21.85 \pm 11.10$ vs. GT: $-0.20 \pm 13,63)$. C levels we observed an incremental tendency in the CG $(28.65 \pm 43.19 \%)$ and a decrease in the GT $(-4.64 \pm 30.12 \%)$ but there were no significant differences between groups. Conclusions: Consuming TTE was effective for increasing T levels but did not improve of WODs in male CF athletes. Conflict of interests: The authors declare no conflicts of interest.

8

Fruits and vegetables home availability, accessibility and intake in European
preschoolers: ToyBox-study

P. de Miguel-Etayo ${ }^{a, b, c}$, I. Iglesia ${ }^{d, e}$, J. Santabárbara ${ }^{f}$, G. Cardon ${ }^{g}$, V. Iotova ${ }^{h},{\text { Koletzko } B^{i}, \text { A. Kotowska }, \text { Y. Mannis }}^{k}$

${ }^{a}$ Growth, Exercise, NUtrition and Development (GENUD) Research Group.

${ }^{b}$ CIBERObn, Instituto de Salud Carlos III. University of Zaragoza, Zaragoza, Spain.

'Child Health \& Care Research. VU University Medical Center, Amsterdam, Holland.

${ }^{\mathrm{d}}$ Growth, Exercise, NUtrition and Development (GENUD) Research Group.

éFundación Instituto de Investigación Sanitaria Aragón (IIS Aragón) y Red de Salud Materno-Infantil (SAMID), Universidad de Zaragoza, Zaragoza, Spain.

fDepartment of Microbiology, Preventive Medicine and Public Health, University of Zaragoza, Spain.

${ }^{9}$ Department of Movement and Sports Sciences, Ghent University, Belgium.

hMedical University Varna, Varna, Bulgary.

'Dr von Hauner Children's Hospital, University of Munich Medical Centre, Munich, Germany.

'The Children's Memorial Health Institute, Warsaw, Poland.

${ }^{k}$ Department of Nutrition and Dietetics, School of Health Science and Education, Harokopio University, Athens, Greece.

Background: In order to understand the etiology of obesity, the dietary habits need to be further investigated. Objectives: To assess the influence of fruits and vegetables availability and accessibility, as predictors of fruits and vegetables intake in European preschoolers. Methods: The present study included 6696 preschool children (52.1\% boys) from six European countries (Belgium, Bulgaria, Germany, Greece, Poland and Spain), 3.5 to 5.5 years of age, that participated in the ToyBox-study. Data on fruits and vegetables availability and accessibility (yes/no) and fruits and vegetables intake (portions/day) were collected via standardized proxy-administered questionnaires. Linear regression model was performed. The adjusted model included socioeconomic status and weight status. Results: In the adjusted model, boys who had fruits and vegetables available at home, consumed 0.34 portions of fruits $(p \leq 0.001)$ and vegetables $(\mathrm{p} \leq 0.001)$ more than the boys who didn't have fruits and vegetables available. Also, girls who had fruits and vegetables available, consumed $0.35(\mathrm{p} \leq 0.001)$ portions of fruits and 0.27 portions of vegetables more than their counterparts. Moreover, boys who had fruits and vegetables accessible, consumed $0.12(p \leq 0.001)$ portions of fruits and $0.08(\mathrm{p} \leq 0.001)$ portions of vegetables more than the boys who didn't have fruits and vegetables accessible. Girls who had fruits and vegetables accessible, consumed $0.08(\mathrm{p} \leq 0.001)$ portions of fruits and 0.06 portions of vegetables $(\mathrm{p}=0.016)$ more than the girls who did not have fruits and vegetables accessible. Finally, in boys the predictor effect of fruits and vegetables availability was higher than in girls in fruits and vegetables intake ( $2=22.8 \%$; $2=18 \%$; respectively). In girls, the predictor effect of fruits and vegetables accessibility was higher than in boys in vegetables intake $(\mathrm{R} 2=12)$. Conclusion: Food availability has a moderate effect on dietary habits in preschoolers. However, home environment characteristics such as 
family rules or parental role modelling should be considered as other potential factors related to food intake in preschoolers. Conflict of interests: Authors declare no conflicts of interest.

\title{
Relationship of bisphenol A (BPA) exposure with dietary quality in schoolchildren
}

\author{
M. Mariscal-Arcas ${ }^{a}$, M. Martínez-Bebia ${ }^{a}$, N. Giménez-Blasi ${ }^{b}$, J.A. Latorre ${ }^{a}$, D. Espino ${ }^{c}$, F. Olea-Serrano ${ }^{d}$ \\ ${ }^{a}$ Department of Food Technology, Nutrition and Food Science. School of Health Science. University of Murcia. \\ Lorca (Murcia), Spain. \\ ${ }^{\mathrm{b}} \mathrm{S}$ chool of Health Science. University of Isabel I. Burgos, Spain. \\ 'School of Physical Education and Sport Sciences, Autonomous University of Chihuahua. Chihuahua, Mexico. \\ ${ }^{\mathrm{d} D e p a r t m e n t}$ of Nutrition and Food Science. School of Pharmacy. University of Granada. Granada, Spain.
}

Background: Young children, whose growth and development are highly dependent on the endocrine system, are particularly vulnerable to endocrine disruptor exposure. Objectives: Measure BPA migration levels from cans, fruit juice bottles/packs, and microwave containers used for food/drinks consumed by a sample of schoolchildren in Spain and to estimate the relationship between their resulting BPA exposure and diet quality. Methods: Participants completed an encoded questionnaire with four sections: participant data; food frequency questionnaire (FFQ); 3xR24h; anthropometric variables; physical activity variables. Weight (kg) was determined using a floor scale (model SECA 872; Hamburg, Germany) barefoot and wearing light clothes. Height (m) was measured with a stadiometer (model SECA 214).Determination of BPA: Canned food, fruit juices, and plastic microwave containers were purchased in Spanish supermarkets. This study focused solely on BPA leaching from the packaging, given that the BPA content of the food itself can derive from processing and other environmental sources. The BPA monomer content was determined with a previously validated methodology. BPA was analysed by HPLC as previously reported and BPA was confirmed by gas chromatography-mass spectrometry (CG/MS) as previously reported for our research group. The limit of quantification for bisphenol A was $2.3 \mathrm{ng} / \mathrm{mL}$. Results: The mean BPA concentration was $11.8 \mathrm{ng} / \mathrm{mL}$ for vegetable cans, $22.1 \mathrm{ng} / \mathrm{mL}$ for pulse cans, $3.6 \mathrm{ng} / \mathrm{mL}$ for juice bottles/packs, and $1.2 \mathrm{ng} / \mathrm{mL}$ for microwave containers. Results revealed a significant association between the Mediterranean Diet and low BPA exposure of the children. BPA exposure below the median level was significantly associated with a higher score in both 7-8yrs $(\mathrm{P}=0.030)$ and $9-10 y r s(\mathrm{P}=0.0001)$ groups. Conclusion: In conclusion, children with a stronger adherence to a Mediterranean-like diet appear to be less exposed to BPA migrating from food packaging and microwave containers. Further research is warranted on the inadvertent exposure of children to endocrine disrupting chemicals from these sources. Conflict of interests: The authors report no conflicts of interest. Acknowledgement: This work was supported by grants from the Spanish Ministry of Health (FEDER-ISCIII PI14/01040).

10

Dietary exposure assessment of pregnant women to Bisphenol A (BPA)

\author{
M. Mariscal-Arcas ${ }^{a}$, J.A. Latorre ${ }^{a}$, M. Martínez-Bebia ${ }^{a},{ }^{\prime}$. Giménez-Blasi ${ }^{b}$, D. Espino ${ }^{c}$, F. Olea-Serrano ${ }^{d}$
}

aDepartment of Food Technology, Nutrition and Food Science. School of Health Science. University of Murcia. Lorca (Murcia), Spain.

bSchool of Health Science. University of Isabel I. Burgos, Spain.

'School of Physical Education and Sport Sciences, Autonomous University of Chihuahua. Chihuahua, Mexico.

${ }^{\mathrm{d} D e p a r t m e n t}$ of Nutrition and Food Science. School of Pharmacy. University of Granada. Granada, Spain.

Background: Bisphenol-A (BPA) has been detected in canned food, among other food containers. In mammal studies, BPA transferred from mother to fetus, caused abnormality of reproductive organs, and advanced female puberty. Objectives: The objectives of this study were to measure BPA migration levels from food cans and microwave containers and to estimate the consequent exposure of pregnant women in Southern Spain to BPA. Methods: BPA from canned food and microwave containers was analyzed using HPLC and gas chromatography mass spectrometry. Population was cohort of mother-son pairs established at the San Cecilio University Hospital of Granada. Frequency of food consumption (including canned food) was studied with a semi-quantitative questionnaire. Results: The most frequently consumed products were fish 
and juice cans, consumed $1-3$ times/week by $34.7 \%$ and $22.3 \%$ of the study population, respectively. The women made little use of polymer microwave containers, $52.8 \%$ never using them and $45.9 \%$ using them $<3$ times/month. Estimated mean (standard deviation) intake of BPA was 1.1(0.839) microgram/day. Conclusion: No relationship was found between BPA exposure and maternal socio-demographic variables or newborn characteristics. This study offers the first estimate of BPA dietary intake by pregnant women in Southern Spain. The consumption of canned foods and drinks by these women means that their exposure was lower than EFSA estimates for the European population. Nevertheless it remains of concern, given the proven undesirable effects of low-level exposure and higher susceptibility of pregnant women. Conflict of interests: The authors report no conflicts of interest. Acknowledgement: This work was supported by grants from the Spanish Ministry of Health (FEDER-ISCIII PI14/01040) and the European Union Commission (QLK4-1999-01422 and FOOD- CT-2003-506319).

11

\title{
Moderate but not high dietary Folic Acid supplementation prevents aging cerebellum alterations in rats
}

M.P. Garzón-Riveros ${ }^{a}$, T. Partearroyo ${ }^{b}$, N. Úbeda ${ }^{b}$, C. Maestro-de-las-Casas ${ }^{a}$, A. Peña-Melián ${ }^{a}$, G. Varela-Moreiras ${ }^{b}$, J. Pérez-Miguelsanz ${ }^{a}$

aDepartamento de Anatomía, Facultad de Medicina, Universidad Complutense, Madrid, España.

bepartamento de Ciencias Farmacéuticas y de la Salud, Facultad de Farmacia, Universidad CEU-San Pablo, Madrid. España.

Background: Cerebellum is a major brain structure particularly sensitive to folate metabolic disturbances, which contributes to the control of voluntary movements, posture, balance and motor learning, as well as cognitive and emotional functions. Objectives: The objective of this study was to investigate the effects of three dietary levels of folic acid (FA) on cerebellar architecture, organization and meningeal disposition in aged rats. Methods: Twenty-five male OFA rats (a Sprague-Dawley strain), 18 month old, were classified into the following dietary groups: FA supplemented ( $8 \mathrm{mg} / \mathrm{kg} \mathrm{FA}, \mathrm{n}=8$ ); FA supra-supplemented (40 mg/kg FA, $n=9$ ); and control ( $2 \mathrm{mg} / \mathrm{kg} \mathrm{FA}, \mathrm{n}=8$ ). Rats were fed ad libitum for 30 days. Results: Microscopic analysis revealed diverse types of defects: i) In the vermis zone, partial disappearance of primary and secondary fissures and/or molecular layer heterotopia. ii) In lateral zones, bilateral partial absence of folia due to the disappearance of the molecular layer. iii) The pia mater was absent in fissure heterotopia and/or delaminated in several layers with affectation of the molecular layer at the surface of the cerebellum. Iv) the presence of numerous dilated veins. The supra-supplemented diet group exhibited the highest incidence of abnormalities (88.9\%) compared with the supplemented diet group ( $\mathrm{p} \leq 0.05$ ). The primary fissure was predominantly affected, sometimes accompanied by defects in the secondary fissure, except in the supplemented group. The pia mater was more altered in the FA supra-supplemented group Conclusion: Our findings show time that moderate but not control or high FA supplementation prevented physiological cerebellum alterations of primary fissure in aged rats. The present study is the first to demonstrate that cerebellar morphological defects can arise from control, as well as high FA levels in the diet, proposing these alterations of the secunda fissure and pia mater be directly related to dietary FA levels. Conflict of interests: Authors declare no conflicts of interest.

12

Relationship between food preferences, parental disputes, hydration status and eating disorder risk in adolescents. UP\&DOWN substudy

\author{
J.A. Rodríguez-Laprast ${ }^{a}$, S. Gómez-Martínez ${ }^{a}$, A. Hernández ${ }^{a}$, V. Cabanas-Sánchez ${ }^{b}$, O.L. Veiga ${ }^{b}$, A. Marcos ${ }^{a}$ \\ anmunonutrition Group. Institute of Food Science, Technology and Nutrition. Spanish National Research \\ Council. Madrid. Spain. \\ ${ }^{\mathrm{b}}$ Department of Physical Education, Sport and Human Movement. Autonomous University of Madrid, Madrid, \\ Spain.
}

Background: Adolescence is a decisive period in which eating habits, including food preferences, are adopted. Likewise, an optimal hydration is essential within thenutritional status. In addition, the role of 
family is a key factor for overall development throughout the life-span and Eating Disorders (ED) are among the most common mental health problems in young population. Objectives: To assess possible relationships between food preferences, parental disputes, hydration status and ED risk. Methods: A cross-sectional substudy from the UP\&DOWN Study was performed. A total sample of 31 girls (mean age $15.44 \pm 1.44$ years) was included. ED risk was measured by the SCOFF questionnaire, a validated screening tool for ED assessment. A healthy food preferences pattern was created with a score choosing the following foods (legumes, fruits, vegetables, fish and dairy products) being positively rated in a 0-5 scale; 1 point was given for each healthy choice. Parental disputes were evaluated using the Lewis stressful events scale. Total Body Water [TBW (L)], related to weight of subjects,was measured by bioimpedance. Results: Almost 39\% of subjects showed ED risk.In a logistic regression model, food preferences (OR=0.49, $\mathrm{P}=0.05)$ and parental disputes $(\mathrm{OR}=4.68, \mathrm{P}=0.03)$ were associated with $\mathrm{ED}$ risk. However, there was no association between TBW and ED risk $(\mathrm{OR}=1.95, \mathrm{P}>0.05)$. A binomial probit model was calculated to study the effect of TBW (L), food preferences and parental disputes on ED risk. An association between unhealthy food preferences and ED risk $(\mathrm{P}=0.02)$ was observed. Conclusion: $\mathrm{n}$ view of these results, girls who showed unhealthy food preferences and felt more affected to parental disputes were more likely to develop ED risk. Therefore, further research concerning the study of food preferences together with specific stressful events such as parental disputes, particularly in female adolescents, would be required in order to prevent ED. Conflict of interests: The authors have no conflicts of interest.

13

\title{
Supplementation with a broccoli extract reduces metabolic syndrome disturbances in rats
}

\author{
P. Aranaz ${ }^{a}$, D. Navarro-Herrera ${ }^{a}$, A. Romo-Hualde ${ }^{a}$, M. Zabala ${ }^{a}$, M. López-Yoldi ${ }^{a}$, J.A. Martínez ${ }^{a}$, F.I. Milagro ${ }^{a}$ \\ ${ }^{a}$ Centro de Investigación en Nutrición, Universidad de Navarra, Pamplona, Navarra, España.
}

Background: The consumption of functional foods and plant-derived bioactive compounds as supplements has emerged as a strategy to manage metabolic syndrome disturbances. In this context, plants from the genus Brassica (Brassicaceae or Cruciferae) are considered attractive functional items for a healthpromoting diet. Objectives: The potential anti-obesity properties of an ethanolic broccoli extract (BE) in a diet-induced obesity model of Wistar rats were investigated. Methods: Two doses $(14$ and $140 \mathrm{mg} / \mathrm{kg}$ animal) of BE were evaluated in high fat/high sucrose diet (HFS)-induced obese Wistar rats. The effects of BE supplementation on body weight gain, food efficiency, adiposity, lipid metabolism, liver steatosis and glucose homeostasis were analysed, including the potential modulatory role on adipocyte metabolism. Results: After 10 weeks of BE supplementation, treated animals exhibited reduced body weight gain and food efficiency, decreased atherogenic index of plasma and improved glucose tolerance in comparison with non-supplemented rats. BE also reduced the fat mass and adipocyte size in the retroperitoneal depot, which was accompanied by the down-regulation of the adipocyte-specific genes Cebpa, Srebp1, Fasn and Adipoq. $\mathrm{BE}$ also decreased liver fat accumulation, which was accompanied by the up-regulation of the lipid-oxidation related genes Acot8 and Acox1, and the down-regulation of the lipogenesis-related genes Fasn, Fatp4 and Srebf1 in hepatocytes. Conclusion: Our data provide new knowledge about the potential role of broccoli components in the prevention and treatment of metabolic syndrome disturbances. Conflict of interests: Authors declare that they have NO affiliations with any organization or entity with financial or non-financial interests in this matter or materials reported in the current abstract.

Vegetarianism and veganism versus mental health and cognitive outcomes. A systematic review and meta-analysis

\author{
I. Azorín ${ }^{a}$, I. Huybrechts ${ }^{b}$, L.A. Moreno ${ }^{a}$, N. Michels ${ }^{c}$ \\ ${ }^{a}$ Growth, Exercise, Nutrition and Development (GENUD), Edificio SAI. Zaragoza, Spain.
}


b International Agency for Research on Cancer, World Health Organization, Lyon, France.

'Department of Public Health, Ghent University, Ghent, Belgium.

Background: Vegetarian and vegan diet become more popular. Although these diets are known to ameliorate health, certain deficiencies might put these people at higher risk. Cognitive and mental health are related diseases with high economic burden. Objectives: A meta-analysis on the relation of vegan or vegetarian diets with cognitive and mental health. Methods: PubMed, Scopus, Science Direct and Proquest databases were examined from the beginning to July 2018. Only original observational or interventional human studies on pure vegan/vegetarian diets (excluding case-reports, studies without omnivorous control group, multi-lifestyle interventions and eating disorders) were selected by two independent reviewers. Raw mean and standard deviation was taken for continuous outcomes while number of events for categorical outcomes. Results: From 1,249 hits, 13 articles were included on total 17,809 individuals. Most studies were cross-sectional, two prospective and three interventional. Six studies included vegetarians, two vegans and five both. Study quality was rather medium. No significant association was found for the continuous depression score $(n=9)$, stress $(n=5)$, well-being $(n=4)$ or cognitive impairment $(n=3)$. Vegans/vegetarians were at increased depression risk (odds ratio $=2.142[1.105,4.148], \mathrm{n}=2$ ) and had lower anxiety scores (mean difference $=-0.847[-1.677,-0.018], \mathrm{n}=7$ ). Heterogeneity was large, thus subgroup analyses showed a lot of contrasting significances with higher mental risks mainly in those under 26y and in higher quality studies but no difference whether vegans versus vegetarians were included. Conclusion: More studies (especially on cognitive health) with overall better quality (e.g. adjusting for confounders) are needed to make clear positive/negative associations.

15

\title{
Culinary Nutrition recipe reformulation process. "Cookies" as an example
}

\author{
M. Casillas-González ${ }^{a}$, B. Beltrán de Miguel $^{a}$
}

${ }^{a}$ Dpto. Nutrición y Ciencias de los Alimentos. Facultad de Farmacia (UCM). Madrid. España.

Background: The purpose of a Culinary nutrition recipe-reformulation is to change an existing recipe into a healthier and more nutritional version while maintaining its sensory attributes (Trakselis and Stein, 2014).

Objectives: Taking into account decreasing total sugar intake in diets is an universal health objective for non-comunicable diseases prevention, four reformulation modalities of a homemade recipe of "cookies" are proposed, with the objective of adapting it to the conditions of a person that requires decreasing sugars content in his diet. Methods: Four dietary reformulations (A, B, C and D) were proposed from the original recipe, focusing on the reduction of simple sugars by replacing white sugar with non-calorie sweeteners, milk chocolate with purer varieties and wheat flour with whole wheat or spelled flour. Results: In the four reformulated recipes a reduction of $48 \%$ in total carbohydrate composition and $85 \%$ in sugar content was achieved, increasing the possibility that people with a more exhaustive control of the consumption of simple sugars could consume cookies (in a moderated quantity). At the same time, an increase in the amount of fiber was also achieved, which tripled the amount present in the original cookie. In addition, total fats were $16 \%$ lower in recipe B compared to the others. In the rest of evaluated nutrients, there were no large differences. Conclusion: The culinary dietary adaptation of the recipes to different physiological or pathological situations and the acquisition by the consumer of the necessary food skills for doing this changes, is a promising tool of health and nutritional education. Conflict of interests: There are no conflicts of interest.

16

\section{Structure, quality and process evaluation of interventions to prevent childhood obesity.}

\author{
M. Seral Cortes ${ }^{a}$, P. De Miguel Etayo ${ }^{a, b, c}$, P. Zapata ${ }^{d}$, LA. Moreno Aznar ${ }^{a, b}$
}

${ }^{a}$ GENUD [Growth, Exercise, Nutrition and Development] Research Group, Facultad de Ciencias de la Salud, Universidad de Zaragoza, Zaragoza, Spain. 
bInstituto Agroalimentario de Aragón [IA2], Instituto de Investigación Sanitaria Aragón [IIS Aragón]. ${ }^{c}$ Centro de Investigación Biomédica en Red de Fisiopatología de la Obesidad y Nutrición (CIBERObn).

dUniversidad de Antioquia. Departamento de Nutrición y Dietética, Medellín, Colombia.

Background: Childhood obesity is one of the most serious global public health challenges of the 21st century. Health and scientific organizations demand early interventions, although their complexity generates difficulties for implementation. Objetivos: The aim of the present study is to evaluate the quality of the studies based on the intervention performed and its relationship to the process evaluation. Methods: We performed a systematic review of randomized control trials aiming to prevent childhood obesity. Interventions are classified either using a three components combination (3CC) of physical activity (PA), diet (D) and behavioral support (BS), a combination of two (2CC) or one component alone. The Cochrane tool for Systematic Reviews was used to assess and report methodological risk of bias. Each item was judged at high (HR), low (LR) or unclear (UN) risk of bias as per criteria. Key domains random sequence generation and allocation concealment domains were also assessed. Process evaluation (PE) was evaluated when at least one PE indicator was adequately implemented. Results: 3CC was the most used approach 16/41. Almost half of studies (17/41) scored HR (3CC 7/16; 2CC 5/9 and PA only 5/12). Only 5/41 studies reported LR (3CC 2/16; 2CC 2/9 and PA only 1/12). Concerning random sequence generation, all types of structures used had no differences (LR 3CC: 15/16; 2CC: 9/9 and PA only: 12/12). Concerning the allocation concealment (LR 3CC intervention (9/16), 2CC intervention (4/9) and PA only (4/12). PE was used in 7 papers (3CC intervention: 2/7; 2CC intervention: 2/7; PA only: 2/7 and BS only: 1/7). Conclusions: 2CC studies seem to have the highest proportion of LR of bias (22,2\%). 3CC showed the lowest LR (56.2\%) in allocation concealment. The 2CC showed the highest proportion of PE $(22,2 \%)$. There could be a relationship between the quality and PE performance. Conflict of interests: Authors declare no conflict of interests.

17

\title{
Relationship between lifestyle and Body Mass Index in mediterranean southeast Spanish university students from the School of Health Science of Lorca (Murcia-Spain)
}

\author{
M. Martínez Bebiáa ${ }^{a}$ N. Gimenez-Blasi ${ }^{b}$, JA. Latorre $^{\mathrm{a}}$, A. Reboredo-González ${ }^{\mathrm{a}}$, F. Olea-Serrano ${ }^{\mathrm{C}}$, M. Mariscal- \\ $\operatorname{Arcas}^{c}$ \\ ${ }^{a}$ Department of Food Technology, Nutrition and Food Science. School of Health Science. University of \\ Murcia. Lorca, Murcia. Spain. \\ ${ }^{b}$ School of Health Science. University of Isabel I. Burgos, Spain. \\ 'Department of Nutrition and Food Science. School of Pharmacy. University of Granada. Granada. Spain.
}

Background: Obesity and overweight are among the most important factors in the appearance of Non Communicable Diseases (NCDs). Knowing and assessing different populations, allows us to determine the differences in factors that affect to the health. Objetivos: To describe the relationship between lifestyle, gender and BMI through an observational, cross-sectional study. Methods: A specific questionnaire was designed, which included questions about lifestyle and exposure estimation to endocrine disruptors (ED). The population was formed by students of the University of Murcia in the degrees of Human Nutrition and Dietetics, and Nursing. The study population included 226 subjects, 164 women $(72.6 \%)$ and 62 men (27.4\%).Mean age was 22.93yrs (SD: 8.213) for women and 22.29yrs (SD: 4.440) for men.The statistical analysis included chi-square test (gender-lifestyle),t-test and ANOVA (lifestyle-BMI) with a confidence level of 95\%. Results: Statistically significant differences were found between gender for:Degree, beer consumption, drugs, low calorie foods, iodized salt, canned corn, microwavable pasta, juice container type, kind of displacement, activity when leaving the school, times brushing teeth and using brackets $(\mathrm{p} \leq 0.05)$.Statistically significant differences were found between lifestyle and BMI for:Sleep hours, consumption of low calorie products, have made a diet to lose weight, frequency of assistance to the dentist, reason for the last time, frequency of brushing, type of toothpaste, rinse with fluoride and use of brackets $(\mathrm{p} \leq 0.05)$. Conclusion: Individuals declare to practice healthy life habits. There are some important elements to consider (consumption of noodles for microwaves, different levels of physical activity between genders, oral care). The low calorie products, the diets, the hours of sleep and the oral care seem to be related to the BMI. Conflict of interests: Authors report no conflicts of interest. Acknowledgement: This work was supported by grants from the Spanish Ministry of Health (FEDER-ISCIII PI14/01040) 


\title{
Evolution of body composition in technified alpine skiers with a Mediterranean diet calorie profile
}

\author{
N. Velasco ${ }^{a}$, C. Monteagudo ${ }^{a}$, A. García-Nieto ${ }^{a}$, P. Moreno ${ }^{a}$, MJ. Jimenez-Casquet ${ }^{b}$, T. Navarro ${ }^{a}$, I. Purcell $^{a}$, \\ O. Dominguez ${ }^{a}$
}

${ }^{a}$ Dpt. Nutrición y Bromatología, Facultad de Farmacia, Universidad de Granada. España.

buniversidad de las Islas Baleares. España.

Background: Sport nutrition in high performance athletes is a discipline in which we try to apply all the nutritional, food science and food technology benefits in the optimization of sports performance through nutritional health of the athlete. The Mediterranean Diet (MD) for Mediterranean athlete should be their adequate nutritional pattern. Methods: Through a nutritional intervention with a recommended MD caloric profile, we pretend to observe evolution in body composition in alpine skiers of the Andalusian Federation of Winter Sports during 2018-2019 season. Results: Study population is $100 \%$ of Technified Alpine Skiers of the Andalusian Federation of Winter Sports with a mean age of 17.5yrs (SD:2.18),83.3\% men and 16.7\% women. Data collection was conducted from October 2018-April 2019. Informed written consent to participation was obtained from the parents/guardians. This sample of adolescents was previously investigated by our group approved by the Ethics Committee of the University of Granada.Weight,height,arm/leg perimeters and body skinfolds were measured according to the international consensus of ISAK.Through these data, we estimated BMI,\%fat, fat weight,\%MLG and MLG-Weight.Diets were carry out based on their personal energy and nutrient needs with a traditional MD caloric profile:55\% of energy from carbohydrates,10\% of energy from proteins and 35\%of energy from lipids.Descriptive study of all measured and estimated variables and Kendall's tau trends test to prove the presence of a trend over time. Mean weight was 67kg (SD:13.30), mean BMI of 21.74kg/m2(SD:2.67),mean \%fat of 11.3\%(SD:1.61). Correlation study of all data measured and estimated at different times of the season,negative correlation was observed $(\mathrm{p}<0.05)$ when comparing their time evolution for the triceps skinfold ( $\mathrm{r}=-0.365, \mathrm{p}=0.024)$, suprailiac $(\mathrm{r}=-0.329, \mathrm{p}=0.044)$, abdominal ( $\mathrm{r}=-0.337, \mathrm{p}=0.037)$, \%fat ( $\mathrm{r}=-$ $360, p=0.025)$. Conclusion: Correlation study of body composition in Mediterranean Technified Alpine Skiers shows a significant improvement carring out a balanced diet according to the traditional MD for such important parameters in an optimal sports performance as triceps, suprailiac and abdominal skinfolds and \%fat. Conflict of interests: Authors report no conflicts of interest. Acknowledgement: This work was supported by grants from the Andalusian Federation of Winter Sports.

\section{Effects of two polyphenol-rich food ingredients, obtained from apple and onion, in the prevention of hepatic steatosis in rats}

\author{
N. Romo-Miguel ${ }^{a}$, S. Gómez-Zorita ${ }^{a, b}$, A. Fernández-Quintela ${ }^{a, b}$, D. González-Peña ${ }^{c}$ B. de Ancos ${ }^{c}$, C. \\ Sánchez-Morenoc, M.P. Portillo ${ }^{a, b}$ \\ ${ }^{a}$ Nutrition and Obesity Group, Department of Nutrition and Food Science, Faculty of Pharmacy, University \\ of the Basque Country (UPV/EHU) and Lucio Lascaray Research Center, 01006 Vitoria, Spain. \\ ${ }^{b}$ CIBERObn Fisiopatología de la Obesidad y Nutrición, Instituto de Salud Carlos III, Madrid, Spain. \\ Institute of Food Science, Technology and Nutrition (ICTAN), Spanish National Research Council (CSIC), \\ ES-28040 Madrid, Spain.
}

Background: Hepatic steatosis, whose prevalence is increasing worldwide, is considered a benign condition, asymptomatic and reversible. However, it can lead to more severe pathologies, such as steatohepatitis, cirrhosis or even cancer. Dietary treatment is critical in the management of steatosis, and polyphenols have been considered as preventive or therapeutic tools. Objectives: To analyse the effect of two polyphenol-rich 
food ingredients (obtained from apple and onion) on hepatic steatosis in obese Zucker $(f a / f a$ ) rats, exploring their mechanism of action. Methods: 24 Zucker $\mathrm{fa} / \mathrm{fa}$ rats were fed a standard diet and divided into 3 groups (n=8): control (C), supplemented with 10\% of an apple ingredient (AI) or an onion ingredient (OI) for 8 weeks. Hepatic triglyceride content, fatty acid synthase (FAS) and carnitine palmitoyl transferase-1a (CPT1a) activities were assessed by spectrophotometry. Acetyl-CoA carboxylase (ACC), fatty acid transport protein 5 (FATP5), aquaporin 9 (AQP9) and mitochondrial transcription factor A (mtTFA) protein expressions were determined by western blot. Results: Hepatic triglyceride content was significantly reduced in OI group compared with C group (48.59 $\pm 0.80 ; 36.57 \pm 1.87)$. Regarding de novo lipogenesis, in AI group but not in OI group, FAS activity $(p<0.05)$ and AQP9 protein expression $(p<0.05)$ were higher compared with control rats, whereas the expressions of the other lipogenic proteins remained unchanged. Regarding fatty acid oxidation, rats fed the polyphenol food ingredient enriched diets showed a tendency to increased) CPT-1a activity $(p<0.1)$ when compared with $\mathrm{C}$ group. Finally, although no statistical differences were observed, the values of mtTFA roughly doubled control values. Conclusion: Onion ingredient, but not apple ingredient, could be useful in the prevention/treatment of hepatic steatosis likely due to the involvement of an increased fatty acid oxidation. Conflict of interests: The authors declare no conflict of interest.

\section{Relationship between energy intake and caloric profile in winter sport federated population}

C. Monteagudo ${ }^{a}$, A. García-Nieto ${ }^{a}$, P. Moreno ${ }^{a}$, MJ. Jimenez-Casquet $^{a}$, T. Navarro ${ }^{a}$, N. Velasco ${ }^{a}$, M. Medina ${ }^{a}$, A. Romero ${ }^{a}$, I. Purcell ${ }^{a}$, M. Mariscal-Arcas ${ }^{a}$

${ }^{a}$ Dpt. Nutrición y Bromatología, Facultad de Farmacia, Universidad de Granada. España.

Background: Adherence to the Mediterranean Diet (MD) of the Spanish population is decreasing nowadays. The aim of this study was to analyse the adherence to the MD in Spanish elite athletes. Methods: From an ongoing study, this is a preliminary analysis of the nutritional evaluation of the federated population in winter sports in Andalusia approved by the Ethics Committee of the University of Granada. 62 subjects ( $51.5 \%$ men, mean age $14.35+/-3.96$ years; $48.5 \%$ women, mean age $19.19+/-5.93$ years) were studied. Data collection was carried out between February and April 2019. For the estimation of energy and nutrients intake FFQ and $24 \mathrm{~h}$ recall were used. Statistical analysis: means, standard deviations, maximum, minimum and $t$ test for mean comparison by gender. Results: We found a mean men's energy intake of 1801.62Kcal/d (SD:421.42) and a mean women's energy intake of 1818.25Kcal/d (SD:599.98). Mean men's caloric profile was $41.24 \%$ energy intake from carbohydrates (SD:6.28), $19.47 \%$ (SD:3.13) from protein and 37.28\% (SD:6.61) from lipids. Women had 36.21\% (SD:7.96) energy intake from carbohydrates, 17.66\% (SD:3.45) energy intake from protein and 43.33\% (SD:6.70) energy intake from lipids. No statistically significant differences between gender were found ( $p>0.05)$. Conclusion: Mean energy intake between men and women was similar. Caloric profile was similar to the general Spanish population reported by other authors, where there is a very high percentage of energy intake from proteins and very low percentage from carbohydrates and can be described as a decrease in adherence to the Mediterranean Diet pattern. This study shows an unbalanced energy intake and caloric profile, not being appropriate for an adequate Mediterranean Diet. Conflict of interests: Authors report no conflicts of interest. Acknowledgement: This work was supported by grants from the Andalusian Federation of Winter Sports(FADI).

Epigenetic regulation in obesity: Interactions between microRNAs and DNA methylation.

F. Salas-Pérez ${ }^{a}$, T. Silveira Assmann ${ }^{a}$, Jl. Riezu-Boj ${ }^{a, b, c}$, ML. Mansego ${ }^{d}$, J. Alfredo Martínez ${ }^{a, b, c, e}$, Fl. Milagro $^{a, b, c}$

aDepartment of Nutrition, Food Science and Physiology, Center for Nutrition Research, University of Navarra, Pamplona, Spain. 
bIdiSNA, Navarra Institute for Health Research, Pamplona, Spain.

'CIBERObn, Fisiopatología de la Obesidad y la Nutrición, Carlos III Health Institute, Madrid, Spain.

${ }^{\mathrm{d}}$ Department of Bioinformatics, Making Genetics S.L, Pamplona 31002, Spain.

Institute IMDEA Food, Madrid 28049, Spain.

Background: Obesity is a multifactorial chronic disease, whose epidemic requires a better understanding of the underlying mechanisms. Epigenetic regulation of gene expression could be a relevant contributor to the obesity susceptibility. MicroRNAs (miRNAs) expression and DNA methylation are two epigenetic mechanisms that regulate gene expression. Moreover, these mechanisms play a pivotal role in the regulation of physiological processes that can vary due to metabolic disturbances. Objective: To analyze the interactions between circulating miRNA expression profile and DNA methylation levels to investigate possible epigenetic regulation of genes involved in metabolic pathways in obese and non-obese subjects. Methods: A total of 103 participants $\left(44.7 \pm 10.1\right.$ years) were grouped according to BMI in obese subjects $\left(30-40 \mathrm{~kg} / \mathrm{m}^{2} ; \mathrm{n}=78\right)$ and non-obese subjects $\left(<25 \mathrm{~kg} / \mathrm{m}^{2} ; \mathrm{n}=25\right)$, recruited in the Metabolic Unit of Nutrition Research Center at University of Navarra. DNA methylation profiles were measured in white blood cells using Infinium MethylationEPIC arrays (Illumina). The levels of 96 miRNAs were quantified in plasma using miRCURY LNA miRNA Custom PCR Panels (Exiqon). Potential target genes of differentially expressed miRNAs were identified in miRWalk3.0 database. Pathway analyses were performed using PathDIP. Statistical significance was accepted when FDR $<0.05$. Results: A total of 26 miRNAs were differentially expressed in plasma of obese subjects compared to non-obese individuals. Bioinformatic analysis of target genes showed that these 26 miRNAs can bind to 1,001 validated genes. On the other hand, a total of 7,467 CpG sites were associated with BMI (adjusted q-value<0.0001) residing in 5,425 genes. Interestingly, the comparison between these two analyses showed 325 common genes that participate in pathways of recognized relevance for metabolic disturbances, such as AMPK, insulin resistance, longevity, TGF-beta, TNF and MAPK signaling pathways.

Conclusion: This analysis evidenced differential miRNA expression profile and DNA methylation relationships of genes involved in metabolic pathways, suggesting a tight regulation in obese subjects, which appears as an interesting approach to find new potential candidate genes for obesity management. Conflict of interests: The authors declare no conflict of interest.

22

\title{
Evolution of body composition in technified beach voley players with a Mediterranean diet calorie profile
}

\author{
JA. Latorre Rodríguez ${ }^{a}$, M. Martínez-Bebiá ${ }^{\prime}$, A. Reboredo ${ }^{a}$, N. Giménez-Blasi ${ }^{b}$, F. Olea-Serrano ${ }^{c}$, M. Mariscal- \\ $\operatorname{Arcas}^{c}$ \\ ${ }^{a}$ Department of Food Technology, Nutrition and Food Science. School of Health Science. University of \\ Murcia. Lorca (Murcia), Spain. \\ ${ }^{b}$ School of Health Science. University of Isabel I. Burgos, Spain. \\ 'Department of Nutrition and Food Science. School of Pharmacy. University of Granada. Granada, Spain.
}

Backgound: Nutritional work on elite athletes includes the intake optimization to facilitate the achievement of an adequate body composition and to sustain the demands of training and competition sessions. This optimized intake can be achieved through the Mediterranean eating pattern. Objectives: To evaluate high level beach volleyball players' body composition evolution, in response to the introduction of intakes adjusted to the Mediterranean diet during 2018-2019. Methods: The evolution of body composition parameters of a female team Beach Volleyball Technification Centre on Lorca-Spain was studied. Mean age was 22.13yrs (SD: 3.13). Data collection was carried out from May 2018 to March 2019. Weight, height, body perimeters and skinfolds were measured according to the International Society for the Advancement of Kinanthropometry (ISAK) method. BMI, \%fat, body fat weight, \%MLG and MLG Weight were estimated. Diets adjusted to the energy and nutrient needs of each athlete were designed, based on the nutritional characteristics of the traditional Mediterranean diet (caloric and lipid profile and recommended food consumption frequency). Descriptive tests of the variables and Kendall's tau test were performed to assess the evolution of body composition parameters. Results: Mean weight was $80.03 \mathrm{~kg}$ (SD: 6.85), mean BMI was $23.33 \mathrm{~kg} / \mathrm{m} 2$ (SD: 0.82 ) and mean fat $\%$ was $13.51 \%$ (SD: 1.01$)$. Negative correlations were found $(\mathrm{p}<0.05$ ) 
for the evolution of the triceps and abdominal skinfolds, $(r=-0.317, p=0.044),(r=-0.522, p=0.001)$, and body fat $\%(r=-0.351, p=0.025)$. Conclusions: Positive evolutions on players' body composition were found when adjusting their intakes to the traditional Mediterranean diet principles. Conflict of interests: Authors report no conflicts of interest. Acknowledgement: This work was supported by Spanish Voleyball Federation (RFEVB).

Inflammatory adipokines are associated with total and abdominal adiposity as early as the prepubertal age in children with obesity

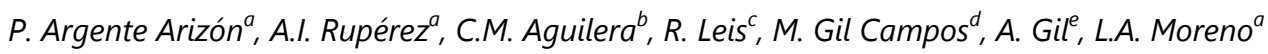 \\ ${ }^{a}$ Growth, Exercise, NUtrition and Development (GENUD) Research Group, Universidad de Zaragoza, \\ Zaragoza, España. \\ ${ }^{b}$ Departamento de Bioquímica y Biología Molecular, Universidad de Granada, Granada, España. \\ 'Departamento de Pediatría, Unidad de Nutrición, Crecimiento y Desarrollo, Universidad de Santiago de \\ Compostela, España. \\ 'Unidad de Investigación Pediatría y Metabolismo, Hospital Universitario Reina Sofía, Córdoba, España. \\ eDepartamento de Bioquímica y Biología Molecular, Universidad de Granada, Granada, España.
}

Background: Obesity is known to be associated with a low-grade inflammatory state, beginning during childhood. However, few studies show whether total and abdominal body fat are associated with proinflammatory biomarkers before puberty. An early identification of these biomarkers could be key to establish primary preventive interventions for the management of inflammation in paediatric obesity.

Objectives: To determine the association between total and abdominal adiposity and a set of inflammatory biomarkers and adipokines before and during puberty. Methods: 1089 children participated in an obesity case-control study (49.4\% males, $69.1 \%$ prepubertals, $25.9 \%$ overweight, $47.4 \%$ obese). The association between total fat (assessed using the BMI, the sum of skinfolds thickness (SF) and fat mass index (FMI) from bioelectrical impedance analysis) and waist circumference as a surrogate marker of abdominal obesity and plasma concentrations of leptin, adiponectin, interleukin 6 (IL-6), interleukin 8 (IL-8), tumour necrosis factor alpha (TNF- $\alpha$ ) and high sensitive C-reactive protein (hsCRP) was assessed with linear regression adjusted for age, sex and recruitment centre, separately for prepubertal children and pubertal children. Results: BMI SF, FMI and WC, were associated with leptin, TNF- $\alpha$ and hsCRP in prepubertal and pubertal children. IL-6 was associated with BMI in prepubertal children and FMI in pubertal children. IL-8 was associated with fat mass in prepubertal children.The strongest observed associations were those between FMI and leptin $(\beta$ 0.698; 95\%CI 1.755, 2.452; $p<0.0001)$ and WC and TNF- $\alpha(\beta$ 0.173; 95\%CI 0.015, 0.035; $\mathrm{p}<0.0001)$ in prepubertal children and FMI and hsCRP $(\beta 0.415 ; 95 \% \mathrm{CI} 0.182,0.472 ; \mathrm{p}<0.0001)$ in pubertal children. Adiposity markers showed a strong negative association with adiponectin in both prepubertal and pubertal children. Conclusion: Plasma concentrations of the analysed biomarkers are related to total and abdominal adiposity, suggesting adipose tissue dysfunction as early as in the prepubertal age. Conflict of interests: Authors report no conflicts of interest.

\title{
Associations between fiber consumption and short chain fatty acids levels and the gut microbiota in apparently healthy adults
}

N. Gonzalez Zancada ${ }^{a}$, N. Redondo Useros ${ }^{a}$, L.E. Díaz $^{a}$, A. Gheorghe ${ }^{a}$, B. Villavisencio ${ }^{a}$, S. Gomez ${ }^{a}$, E. Nova $^{a}$, A. $\operatorname{Marcos}^{a}$

${ }^{a}$ Departamento de Metabolismo y Nutrición Instituto de Ciencia y Tecnología de los Alimentos y Nutrición

(ICTAN), Madrid, Spain. 
Background: The promotion of beneficial commensal bacteria in the gut, along with an increased short chain fatty acid (SCFA) production, have been postulated as potential mechanisms involved in the beneficial effects of a high fiber intake on health. Objectives: To assess the influence of fiber consumption on SCFA levels and the gut microbiota in healthy adults. Methods: 261 adults aged between 25-50 y, not suffering chronic diseases or following medical treatment were included (51\% males) in this study. Fiber intake was assessed by a food frequency questionnaire, and the following fiber consumption (FC) groups were considered: Low (LFC; <15g/day), Medium (MFC; 15-25g/day) and High (HFC); $>25 \mathrm{~g} /$ day). SCFA levels were analyzed by gas chromatography, and gut microbiota composition through 16S rRNA gene amplicon sequencing (V3+V4 gene regions. MiSeq 2x270 Illumina) and taxonomic analysis. To evaluate fiber consumption effects, general linear models were used for SCFA analysis, Kruskal-Wallis test with Bonferroni correction using a false discovery rate (FDR) of 0.10 for microbiota analysis, and Spearman correlations for associations among variables. Results: The HFC group showed higher Clostridiaceae $(\mathrm{P}=0.003)$ and Clostridium levels $(\mathrm{P}=0.004)$ compared to the LFC group. Indeed, these bacterial groups showed a positive correlation with total fiber intake (all $\mathrm{P}<0.001)$, particularly with fiber from vegetables $(\mathrm{P}=0.001$ and $\mathrm{P}=0.005$, respectively) and fruits (only Clostridiaceae, $\mathrm{P}=0.002$ ). SCFA levels were not related to high fiber intake, but the MFC group showed higher acetic and butyric levels compared to the LFC group ( $\mathrm{P}=0.020$ and $\mathrm{P}=0.032$, respectively). Acetic and butyric acids were negatively correlated to Clostridiaceae levels $(\mathrm{P} \leq 0.001)$ but positively to Roseburia, Faecalibacterium and F. praunsnitzii levels (all $\mathrm{P}<0.001$ ). Conclusion: A high intake of fiber might influence Clostridiaceae and Clostridium levels, which seem to be dependent on the type of fiber source. Further research is needed to ascertain other factors related to SCFA production in healthy adults. Conflict of interests: Authors report no conflicts of interest.

\title{
Effect of comorbidity and weight in the influence of lifestyle in quality of life: a pilot study
}

\author{
R. Suarez del Villar Carrero ${ }^{a}$, D. Martinez Urbistondo ${ }^{a}$, M. Agud Fernández ${ }^{a}$, E. Fernández Alonso ${ }^{a}$, L. Prosper \\ Ramos $^{a}$, A. Dominguez Calvo ${ }^{a}$, A. Rodriguez Cobo ${ }^{a}$
}

${ }^{a}$ Dpto. Medicina Interna. HM Sanchinarro. HM Hospitales. Madrid. Spain.

Background: Quality of life (QoL) is a matter of concern in both healthy and diseased individuals. Lifestyle factors such as dietary intake, physical activity and sleep have a direct impact on QoL scales and body weight. In this context, interactions between lifestyle factors and QoL might be different in comorbid and non-comorbid patients. Objectives: To evaluate the influence of lifestyle on quality of life depending on comorbidity and body weight. Methods: Prospective collection of 132 outpatients from an Internal Medicine Service. Validated questionnaires were used to collect BMI, lifestyle and QoL (SF-36) data. Comorbidity was gathered afterwards by the treating physician using the Charlson Comorbidity Index. SPSS v20.0 was used for statistical analysis. As hypothesized, healthy subjects had higher QoL score than comorbid subjects $(\mathrm{p}<0.05)$. When categorizing QoL results in 4 groups $(0-25,26-50 \%, 51-75 \%$ and $76-100$ $\%)$, BMI was associated to a better QoL in both non-comorbid and comorbid groups $(\mathrm{p}<0.05)$. Physical activity and sleep were statistically significant and associated to a better QoL in comorbid patients $(\mathrm{p}<0.05)$. Interestingly, only in those subjects without comorbidities, an association was found between QoL, Mediterranean dietary pattern and physical activity $(\mathrm{p}<0.05)$. Results: A sensitivity analysis in noncomorbid subjects showed interactions of body weight concerning lifestyle features and modifying QoL. Indeed, physical activity and mediterranean diet adherence were directly correlated to a better QoL in non overweight subjects. Instead, in the overweight group, adequation to mediteranean dietary pattern and physical activity had an unconsistent distribution through subgroups of QoL. Conclusions: Comorbidities and overweight need to be assessed in order to interpret the interactions between lifestyle and QoL to provide precision nutrition and health counselling. Conflict of interests: Authors report no conflicts of interest. 


\title{
Fiber and fats intake is related to the gut microbiota composition in apparently healthy adults in a gender-dependent way
}

\author{
N. Redondo Useros ${ }^{a}$, N. González ${ }^{a}$, LE. Diaz ${ }^{a}$, A. Gheorghe ${ }^{a}$, B. Villavisencio ${ }^{a}$, S. Gómez ${ }^{a}$, JA. Celada ${ }^{a}$, A. \\ Marcosa $^{a}$, E. Nova ${ }^{a}$ \\ ${ }^{a}$ Departamento de Metabolismo y Nutrición. Instituto de Ciencia y Tecnología de los Alimentos y \\ Nutrición (ICTAN). Madrid. España.
}

Background: The study of particular nutrients with modulatory properties on the gut microbiota composition and functionality, such as fat and fiber, has gained special attention mainly due to the rise of metabolic diseases in apparently healthy adults on which dysbiosis and immune impairment are often established. Objectives: To assess the influence of fat and fiber consumption on anthropometric measurements, short chain fatty acids (SCFA) levels and gut microbiota in apparently healthy adults. Methods: 261 adults aged between 25-50 y, not suffering chronic diseases or following medical treatment were included ( $51 \%$ males). Fiber and fat intakes ( $\mathrm{g} /$ day) were assessed by a food frequency questionnaire, anthropometric parameters by bioimpendance analysis, SCFA levels by gas chromatography, and gut microbiota composition through 16S rRNA gene amplicon sequencing (V3+V4 gene regions. MiSeq 2x270, Illumina). Spearman correlations were used to evaluate possible associations among variables. Results: Total fiber intake was negatively associated with total dietary fat, saturated and monounsatured fatty acids $(\mathrm{P}<0.001)$. In addition, total fiber $(\mathrm{P}=0.001)$, fiber from vegetables $(\mathrm{P}=0.038)$ and fiber from fruits $(\mathrm{P}=0.004)$ were positively correlated to Coprococcus, which in turn was also negatively associated with total dietary fat $(\mathrm{P}<0.001)$. F. prausnitzzi negatively correlated to saturated fatty acids $(\mathrm{P}<0.001)$ and positively with fiber from grains $(\mathrm{P}=0.022)$ only in women. Body fat and visceral fat were negatively correlated to fiber $(\mathrm{P}=0.002$ and $\mathrm{P}=0.006)$ and positively to Lactobacillus in men $(\mathrm{P}=0.016$ and $\mathrm{P}=0.020)$ and with Clostridium in women $(\mathrm{P}=0.025$ and $\mathrm{P}=0.028)$. No correlations were found between SCFA and diet or anthropometric parameters. Conclusions: Fiber intake seems to be related to beneficial microbes for human health and to a lower fats intake. Further studies need to be separately performed in men and women in order to ascertain the role of particular microbes on body fat deposition. Conflict of interests: Authors report no conflicts of interest.

\section{Uric acid mediates the association between sugar-sweetened beverages consumption and blood pressure in Spanish children.}

G. Pérez Gimeno ${ }^{a}$, Al Rupérez ${ }^{a, b, c}, R$. Vázquez-Cobela ${ }^{d}, R$. Leis ${ }^{d, e}, M$. Gil-Campos ${ }^{e, f}, C M$. Aguilera ${ }^{e, g}, A . G^{e}{ }^{e, g}, L A$. Moreno $^{a, b, c, e}$

${ }^{a}$ GENUD Research group, Universidad de Zaragoza and Food and Agriculture Institute of Aragón (IA2), Zargoza, Spain.

${ }^{\mathrm{b}}$ Health Research Institute of Aragón (ISS Aragón), Zaragoza, Spain.

${ }^{\mathrm{c}}$ Food and Agriculture Institute of Aragón (IA2).

${ }^{\mathrm{d}}$ Galicia Nutrition, Growth and Human Development Research Unit, Pediatric Department; Instituto de Investigación Sanitaria de Santiago de Compostela (IDIS); Universidad de Santiago de Compostela, Spain. eSpanish Biomedical Research Centre in Physiopathology of Obesity and Nutrition (CIBERObn), Instituto de Salud Carlos III (ISCIII), Madrid, Spain.

${ }^{\mathrm{f}}$ Paediatric Research and Metabolism Unit, Hospital Universitario Reina Sofia; Instituto Maimónides de Investigación Biomédica (IMBIC), Córdoba, Spain.

${ }^{9}$ Department of Biochemistry and Molecular Biology II, Instituto de Nutrición y Tecnología de los Alimentos, Universidad de Granada; Instituto de Investigación Biosanitaria ibs, Granada, Spain.

Background: In the last decades, both sugar-sweetened beverages (SSB) consumption and blood pressure (BP) levels are on the rise. There is previous evidence on the association between SSB consumption and elevated BP, as well as with elevated uric acid (UA) serum concentrations. Objectives: For these reasons, the aim of the study is to ascertain whether UA acts as a mediator in the association between SSB consumption 
and BP. Methods: We studied 633 children (5-18 years old, (46.8\% male, 50.2\% prepubertal, $71.6 \%$ overweight/obesity). Food consumption data were obtained from a food frequency questionnaire (FFQ) and the total SSB consumption was calculated as a sum of all beverages that contained sugar. Weight and height were measured and serum UA was analyzed. BP was measured with an electronic manometer; to find a $28.4 \%$ of children with a systolic BP above 90th percentile and $16 \%$ with a diastolic BP above 90th percentile (Task Force 2017). To test the mediation effect of UA on the relationship between SSB consumption and BP levels, the macro PROCESS developed by Hayes was employed, adjusting for BMI Zscore and stratifying the analysis by sex and pubertal status (prepubertal and pubertal). Results: SSB consumption had a significant effect on UA concentrations in pubertal boys $(a=0.0124, S E=0.0059$, $\mathrm{p}=0.0365)$. Also, the mediator variable UA was significant predictor of elevated SBP in pubertal boys $(b=$ 3.1159, $\mathrm{SE}=0.9461, \mathrm{p}=0.0013$ ). In pubertal boys, the assessment of the indirect effect of UA on SSB consumption and SBP levels was significant (indirect effect, $=0.0385 ; 95 \%$ Confidence Interval 0.0080 0.0968). No indirect effect was found in prepubertal or pubertal girls neither in prepubertal boys. Conclusions: UA appears as a mediator in the association between SSB consumption and systolic PB in pubertal boys. Supporting the interest of UA as a marker of diet-induced BP complications. Conflict of interests: Authors report no conflicts of interest.

28

\title{
Body mass index and body fat distribution are related to Firmicutes and Bacteroidetes genera in apparently healthy adults
}

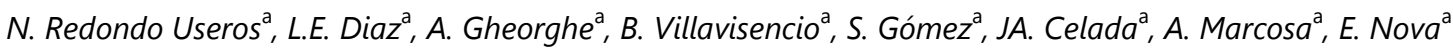 \\ ${ }^{a}$ Departamento de Metabolismo y Nutrición. Instituto de Ciencia y Tecnología de los Alimentos y \\ Nutrición (ICTAN). Madrid. España.
}

Background: The ever more frequent increase of overweight and obesity among apparently healthy adults raises the need to study the interactions among diet, gut microbiota and metabolism in order to prevent metabolic disturbances in the long term. Objectives: To evaluate associations between the body mass index (BMI) and the gut microbiota and short chain fatty acid (SCFA) levels in apparently healthy adults. Methods: The study included 152 adults (51\% males) aged between 25-50 years, not suffering chronic diseases or following medical treatment, who were divided by BMI status into three groups: 1 ) Normoweight ( $\mathrm{n}=57$, $B M I=20-25 \mathrm{~kg} / \mathrm{m} 2) ; 2)$ Overweight $(\mathrm{n}=79, \mathrm{BMI}=25-30 \mathrm{~kg} / \mathrm{m} 2)$ and 3) Obese $(\mathrm{n}=16, \mathrm{BMI}=30-35 \mathrm{~kg} / \mathrm{m} 2)$. Body fat and visceral fat were analyzed by bioimpendance analysis, SCFA levels by gas chromatography and the gut microbiota through $16 \mathrm{~S}$ rRNA gene amplicon sequencing (V3+V4. MiSeq 2x270, Illumina). Firmicutes and Bacteroidetes phyla and genera were studied. Differential abundance among groups was analyzed by Kruskal Wallis and Man-Whitney U tests (q values by the Storey method), SCFA differences by general linear models and variable associations by Spearman tests. Results: Firmicutes and Bacteriodetes abundance was similar among BMI groups. However, obese subjects showed lower levels of the Firmicutes members Lachnospira and Lysinobacillus ( $\mathrm{q}=0.027$ and $\mathrm{q}=0.009$ ), and higher Lactobacillus and Weissella (both $q=0.006)$ compared to normoweight. The Bacteroidetes Hymenobacter $(q=0.005$ and $q=0.010)$, Pedobacter $(\mathrm{q}=0.004$ and $\mathrm{q}=0.006)$ and Candidatus Amoebophilus ( $\mathrm{q}=0.010$ and $\mathrm{q}=0.020$ ) were lower in overweight and obese compared to normoweight. Bacteroidetes members, Lachnospira and Lysinobacillus negatively correlated to visceral fat and waist circumference (all $\mathrm{P}<0.05$ ), whereas Lactobacillus was positively associated to them $(\mathrm{P}=0.003$ and $\mathrm{P}=0.008$, respectively). Obese subjects showed a trend to lower levels of butyric and propionic acids compared to normoweight $(\mathrm{P}<0.1)$. Conclusions: Specific Firmicutes and Bacteroidetes genera are related to visceral fat and a deeper study at species level is warranted. Conflict of interests: Authors report no conflicts of interest. 


\title{
Update of a food composition database of cereal-based gluten-free products commercialized in Spain
}

\author{
MP. González González ${ }^{a}$, V. Fajardo ${ }^{a}$, M. Martínez ${ }^{a}$, L. Samaniego-Vaesken $^{a}$, M. Achón- Tuñón ${ }^{a}$, N. Úbeda $^{a}$, \\ E. Alonso-Aparte ${ }^{a}$
}

${ }^{a}$ Department of Pharmaceutical and Health Sciences, Faculty of Pharmacy, San Pablo-CEU University. Madrid, Spain.

Background: Gluten-free products' manufacturing and consumption are substantially growing, albeit data on their nutritional composition is still limited. Objectives: To update a nutritional composition database including cereal-based gluten-free products, commercialized in Spain. Methods: The original database was constructed in 2016. The update was conducted between December 2018 and March 2019. Products were included when cereal based and carrying the cross-grained symbol. Main commercial and distribution brands were selected. Data were obtained from manufacturer's web pages and food labels. According to declared labels, products were categorized based on LanguaLTM Thesaurus 2017 (EUROFIR classification).

Results: The database includes 730 cereal-based gluten-free foodstuffs, more than three-fold those recorded in 2016. A $10 \%$ of them are no longer available. Products were classified into 8 subgroups. The main one was "fine bakery ware" (N=226; 31.1\%), followed by "prepared food products" ( $\mathrm{N}=155 ; 21.3 \%)$, "bread and similar products" ( $\mathrm{N}=110 ; 15.1 \%)$. Minor categories found were "alcoholic beverages" ( $\mathrm{N}=14 ; 1.9 \%)$, "imitation milk products" $(\mathrm{N}=10 ; 1.4 \%)$ and "frozen dairy desserts" $(\mathrm{N}=9 ; 1.2 \%)$. The subgroup that presented a higher amount of energy, total fats, saturated fats and sugars per $100 \mathrm{~g}$ was "fine bakery ware", with $435.8 \pm 75.5 \mathrm{~g} / 100 \mathrm{~g}, 21.0 \pm 10.7 \mathrm{~g} / 100 \mathrm{~g}, 8.6 \pm 6 \mathrm{~g} / 100 \mathrm{~g}$ and $22.39 \pm 12.38 \mathrm{~g} / 100 \mathrm{~g}$, respectively. Highest fiber, protein and salt values were found in the "bread and similar products" group, with $4.87 \pm 2.77$ g/100 g, $7.6 \pm 3 \mathrm{~g} / 100 \mathrm{~g}$ and $1.5 \pm 0.64 \mathrm{~g} / 100 \mathrm{~g}$, respectively. Micronutrient content was not declared on the label in hardly any gluten-free product. Conclusion: In accordance to the current nutrition labelling from cereal-based gluten-free products, the macronutrient profile can be known, but there is no information on micronutrients. It is urgent to provide vitamin and mineral contents, for a better nutritional assessment of the celiac population. Conflict of interests: Authors report no conflicts of interest.

\section{Olive Pomace oil: an unforgotten source of vitamin E}

\author{
S. González-Rámila ${ }^{a}$, B. Sarriá Ruiz ${ }^{a}$, J. Gómez Cordero ${ }^{a}$, L. Bravo Clemente ${ }^{a}$, R. Mateos Briz ${ }^{a}$ \\ aDepartamento de Metabolismo y Nutrición, Instituto de Ciencia y Tecnología de Alimentos y Nutrición \\ (ICTAN-CSIC).
}

Background: Olive-pomace oil (OPO), obtained from the solid by-product generated during olive oil extraction, is nutritionally relevant due to its high oleic acid (C18:1) content and a wide range of minor bioactive components, such as the powerful antioxidant vitamin E. In Europe, EFSA established the recommended intake of vitamin $E$ for adults at 13 and $11 \mathrm{mg} /$ day for men and women, respectively. As worldwide dietary values are below these recommendations ${ }^{1}$, strategies to cover the daily recommendations of this vitamin are essential. Therefore, this situation represents an opportunity for OPO. Methods: We have carried out a human study to evaluate the possible beneficial role of OPO on cardiovascular health and associated pathologies (hypertension, diabetes and obesity) in comparison with high oleic sunflower oil (HOSO). Volunteers were recruited attending to their cholesterol levels and then, a randomized, cross-over, controlled clinical trial was carried out in 64 subjects $[34$ normocholesterolaemic $(<200 \mathrm{mg} / \mathrm{dL})$ and 30 hypercholesterolaemic (200-280 mg/dL) men and women, aged $18-55 y$ with BMI $\left.18-30 \mathrm{~kg} / \mathrm{m}^{2}\right]$, consisting in two 4-week interventions with OPO and HOSO preceded each by 3-week run-in/wash-out steps. Participants consumed daily $50 \mathrm{~mL}$ of OPO or HOSO, containing 357 and $420 \mathrm{ppm}$ vitamin E, respectively, to meet the nutritional requirements of fat intake. Dietary suggestions will be provided to help volunteers achieve the recommended intake of monounsaturated fat equivalent to $20 \%$ ( $50 \mathrm{~mL}$ of OPO dairy). The consumption of nuts, avocado, olives and other dietary fat different from that the provided in the study was restricted. Dietary and lifestyle habits will be controlled during the study. Intake of macronutrients and micronutrients 
(vitamin E) was analyzed using DIAL software (Faculty of Pharmacy, Complutense University of Madrid). Results: Results showed an increased average vitamin E consumption from $9.61 \mathrm{mg} /$ day in the run-in period to $17.76 \mathrm{mg} /$ day and $18.45 \mathrm{mg} /$ day after OPO and HOSO, respectively. Sensory analysis showed that consumers preference for OPO was higher than that for HOSO. Conclusions: OPO was well accepted and, thus, constitutes an important dietary source of vitamin E covering the recommended daily intake. It is worth considering OPO as part of a healthy diet. Conflict of interests: Authors report no conflicts of interest. Acknowledgement: This study has been funded by the Interprofesional del Aceite de Orujo de Oliva (ORIVA).

31

\title{
Silicon upregulates the expression of tight junction proteins improved the diabetes- induced intestinal barrier dysfunction in a rat model
}

\author{
M. Hernández-Martin ${ }^{a}$, A. Garcimartín ${ }^{a}$, A. Macho-González ${ }^{b}$, MJ. González-Muñoz ${ }^{c}$, S. Batida ${ }^{b}$, J. Benedí, \\ FJ. Sánchez-Muniz ${ }^{b}$
}

aDepartamento de Farmacología, Farmacognosia y Botánica. Facultad de Farmacia. Universidad Complutense de Madrid. Madrid. Spain.

${ }^{\mathrm{b}}$ Departamento de Nutrición y Ciencia de los Alimentos. Facultad de Farmacia. Universidad Complutense de Madrid. Madrid. Spain.

'Departamento de Ciencias Biomédicas. Unidad Docente de Toxicología. Universidad Complutense de Madrid. Madrid. Spain.

Background: The precise regulation of the intestinal barrier is mandatory for the maintenance of intestinal homeostasis and the body health. The impairment of intestinal barrier function due to the altered assembly of epithelial cell tight junction proteins (T) has been observed in type 2 diabetes (DM), resulting in increased permeability to luminal endotoxins. The endotoxin moves into the blood stream leading to a systemic immune response. Silicon ( $\mathrm{Si}$ ) is an essential micronutrient which exerts various physiological benefits including anti-oxidant, anti-inflammatory and anti-diabetic effects. Objectives: The present study evaluates the positive effects of $\mathrm{Si}$ on colonic barrier integrity by measuring the ocluddin (OC) and zonulin-1 (ZO-1) protein expressions in the colonic mucosa in type 2 diabetic rats. Methods: A saturated-fat hypercholesterolemic diet combined with low-dose Streptozotocin plus nicotinamide was used to induce DM. Morphometric and histological analysis of proximal colonic mucosa were carried out by haematoxylineosin staining. The expression of both OC and ZO-1 was determinate by immunohystochemistry. Results: Streptozotocin-induced diabetes in DM group generated pronounced decreases of the colon weight, cryptlength and increases of the wall thickness, wall cross-sectional area and crypt-width in the proximal colon. Si supplementation led to enhance of intestinal crypt/mucosa structure and the morphometric parameters reaching non-diabetic values. Si increased the immunoreactivity of the both OC and ZO-1 protein expression, improving the protective function of mucosal barrier. Also we found a differentiate immunolocation of these TJ along crypts. The OC and ZO-1 translocation in the crypt could mainly result in a decrease permeability which could avoid endotoxin increase in plasma. Conclusion: these results demonstrate the increase in TJ protein assembly and intestinal TJ integrity by Si. Dietary supplementation of Si could be advantageous as a new therapeutic target to reverse metabolic disorders, such as DM, associated with intestinal barrier dysfunction. Conflict of interests: Authors report no conflicts of interest. 


\title{
Assessment of dairy consumption in 7 years old children in Zaragoza, Aragón, Spain
}

V. Vucinic ${ }^{a}$, I. Iglesia ${ }^{a, b, c}$, M.L. Miguel-Berges ${ }^{a, b}$, P. Flores $^{a b}$, L.A. Moreno ${ }^{a, b, d}$, G.Rodríguez-Martínez ${ }^{a, b, c, e, f}$,

${ }^{a}$ Growth, Exercise, Nutrition and Development (GENUD) Research Group, Universidad de Zaragoza.

Zaragoza. Spain.

${ }^{\mathrm{b}}$ Instituto de Investigación Sanitaria Aragón (IIS Aragón). Zaragoza. Zaragoza. Spain.

${ }^{c}$ Red de Salud Materno Infantil y del Desarrollo (SAMID), Instituto de Salud Carlos III. Madrid. Spain.

${ }^{d}$ Ciber Fisiopatología de la Obesidad y Nutrición (CIBERObn), Instituto de Salud Carlos III, Madrid. Spain.

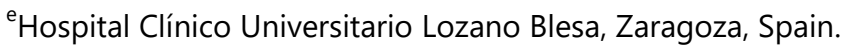

fDepartment of Pediatrics, Universidad de Zaragoza, Spain.

Background: Dairy products are an important dietary source of multiple nutrients for children whereas their consumption is decreasing in recent years. Objectives: To assess differences in consumption of dairy products between girls and boys in children of $7 \mathrm{y}$ with contribution to the percentage of children meeting or not daily dairy recommendations. Methods: In 2016 and 2017 a subsample of 363 children (52\% boys) from the original 1,659 belonging to the birth longitudinal cohort study Growth and Feeding during Lactation and Early Childhood in Children of Aragon (CALINA) was evaluated in a follow up study. Dietary intake was assessed by a semi-quantitative food frequency questionnaire for children (FFQ) filled in by parents. Dairy products were sorted into 6 categories. Results: In total, 26\% $(n=96)$ consume less than 2.0 servings of dairy products per day, which is under the recommendations (2-4 servings/day in Spain). Boys drank significantly more milk compared to girls $(\mathrm{p}<0.029)$. When comparing boys between group meeting and not meeting the recommendations significant difference was not found in the consumption of yogurt $(p<0.101)$ and comparing girls within the groups significant differences were not found between the consumption of yogurt and cheese $(\mathrm{p}<0,055 ; \mathrm{p}<0,646)$. When comparing boys and girls within groups, boys not meeting the recommendations drank significantly more milk than girls $(\mathrm{p}<0.055)$, and in the group reaching the recommendations, boys consumed significantly more cheese $(\mathrm{p}<0.00)$ and girls significantly more sweetened dairy $(\mathrm{p}<0.039)$ than their counterparts. Conclusion: Every child consumed at least one dairy product per day. However, one third of the sample does not meet the current dairy recommendations. When considering all the sample boys consumed more milk than girls, while boys meeting the recommendations consumed more cheese and less sweetened dairy than girls from the same group. Further research is needed to clarify later health implications. Conflict of interests: Authors report no conflicts of interest.

\section{Relationship between screen time and other lifestyle factors of Spanish schoolchildren}

\author{
A. Peral-Suárez ${ }^{a}$, E. Cuadrado-Soto ${ }^{a}$, A.M. López-Sobaler ${ }^{a, b}$, M. Bermejo-López ${ }^{a, b}$, M.C. Torquemada ${ }^{a}$, J.M. \\ Perea-Sánchez ${ }^{b, c}$, R.M. Ortega $a^{a, b}$ \\ ${ }^{a}$ Department of Nutrition and Food Science. Faculty of Pharmacy at Complutense University of Madrid. \\ Madrid. Spain. \\ ${ }^{\mathrm{b}}$ Research group VALORNUT-UCM (920030). Faculty of Pharmacy at Complutense University of Madrid. \\ Madrid. Spain. \\ ${ }^{\mathrm{C}}$ Faculty of Health Science. University Alfonso X El Sabio. Madrid. Spain.
}

Background: Since technology development, some sedentary behaviours such as recreational use of electronic devices has become more popular between children. Time spent in this kind of activities may negatively affect other aspects of children lifestyle. Objectives: The aim of this study was to relate children screen time to other factors of lifestyle, such as sleep, active play or diet quality. Methods: A sample of 359 children (178 girls) aged 7-11 years from 6 Spanish provinces was studied. A daily physical activity questionnaire was used to obtain the daily screen time (ST), sleep duration (SD) and active play time. Children were divided into two groups depending on their adherence to Canadian sedentary guidelines: Non-adherence (NA) (ST $>2 \mathrm{~h} /$ day) or good adherence (GA) (ST $\leq 2 \mathrm{~h} /$ day). To assess the diet quality, the children completed a 3-days food record with their parents help, including one weekend day. DIAL software 
was used to process this questionnaire and estimate the Healthy Eating Index (HEI). For statistical analysis, Student's T test and Mann Whitney's U test (non-parametrical variables) were used to compare means, and Spearman's Rho was used for correlations. Results: The mean ST was $2.41 \pm 1.16 \mathrm{~h} /$ day and the percentage of adherence to sedentary guidelines was $58.05 \%$ with no significant differences among sexes. ST of children correlated negatively to HEI $(\mathrm{r}=-0.129 ; \mathrm{p}<0.05)$ and SD $(\mathrm{r}=-0.125 ; \mathrm{p}<0.05)$. Furthermore, GA group had higher HEI $(58.54 \pm 9.75$ vs. $56.22 \pm 9.95 ; p<0.05)$ and higher SD $(9.86 \pm 0.52$ vs. $9.73 \pm 0.59 ; p<0.05)$ than NA group. No differences were found in case of active play time. Conclusions: Screen time is related to lower diet quality and lower sleep duration in children, which can result in a less healthy lifestyle. Conflict of interests: Authors report no conflicts of interest.

34

\section{Relationship between sleep duration and sodium intake assessed by urinary excretion over $\mathbf{2 4} \mathrm{h}$ in Spanish schoolchildren}

E. Cuadrado-Soto ${ }^{a}$, A. Peral-Suárez ${ }^{a}, E_{\text {. Rodriguez-Rodríguez }}{ }^{b, c}$, L.M. Bermejo-López $^{a, b}$, M.D. Salas-González ${ }^{a}$, A. Aparicio $^{a, b}$, R.M. Ortega ${ }^{a, b}$, A.M. López-Sobaler ${ }^{a, b}$.

${ }^{a}$ Department of Nutrition and Food Science, Faculty of Pharmacy, Complutense University of Madrid, Madrid, Spain.

${ }^{b}$ Research Group VALORNUT-UCM (920030), Faculty of Pharmacy, Complutense University of Madrid, Madrid, Spain.

'Department of Chemistry in Pharmaceutical Sciences, Analytical Chemistry, Faculty of pharmacy, Complutense University of Madrid, Madrid, Spain.

Background: Excessive sodium intake and short sleep duration have been associated with cardiovascular risk factors in children. However, it is unknown whether both variables are related to each other. Objectives: To determine the relationship between sodium intake assessed by urinary excretion of sodium over 24-h and sleep duration in Spanish schoolchildren. Methods: 289 schoolchildren (134 girls) aged 7-11 years from five Spanish regional communities were studied. Sleep duration was collected using a daily activity questionnaire. Sodium intake was estimated by measuring sodium excretion in urine for $24-\mathrm{h}$. The population was stratified into two groups taking into account whether the duration of sleep was $\leq 10 \mathrm{~h} /$ day or higher (>10h/day). Spearman's Rho test (rS) was used for correlation, Mann Whitney's U test for means comparison and a logistic regression model to calculate the corresponding odds ratio (OR) and 95\% confidence intervals (CI). Results: $86 \%$ of schoolchildren had sodium intakes greater than $2000 \mathrm{mg}$ $(3113 \pm 1138 \mathrm{mg} /$ day) and $49.3 \%$ of schoolchildren reported sleeping less than $10 \mathrm{~h} /$ day on weekdays $(9.7 \pm 0.6 \mathrm{~h} /$ day). Analyzing weekdays, sodium intake was lower in the group of children with longer sleep duration $(3268 \pm 1101$ vs. $2965 \pm 1169 \mathrm{mg} /$ day; $\mathrm{p}<0.05)$. Furthermore, there was a negative correlation $(\mathrm{rS}=-$ $0.126 ; \mathrm{p}<0.05$ ) between sodium intake and sleep duration. Sleep less than $10 \mathrm{~h} /$ day on weekdays was associated with an increased risk of excreting sodium over $3000 \mathrm{mg} /$ day after adjusting for sex and age (OR: 1.911; 95\% CI: 1.182-3.089; p<0.01). These differences did not appear on weekend days, as children sleep duration was longer $(10.1 \pm 0.8 \mathrm{~h} /$ day $)$. Conclusions: The present findings show that sodium intake is negatively associated with sleep time on weekdays in schoolchildren. Investigation of the mechanisms involved and the potential impact of this relationship on health is warranted. Conflict of interests: Authors report no conflicts of interest. 


\title{
Assessing the potential synergistic effect of soluble dietary fiber and polyphenols in overweight/obese subjects
}

\author{
L. Bravo Clemente ${ }^{a}$, B. Sarriá Ruiz ${ }^{a}$, J. Sarriá Ruiza ${ }^{a}$ S. González Rámila ${ }^{a}$, R. Mateos Briz ${ }^{a}$ \\ ${ }^{a}$ Departamento de Metabolismo y Nutrición, Instituto de Ciencia y Tecnología de Alimentos y Nutrición \\ (ICTAN-CSIC), Madrid, España.
}

Background: Obesity is a major public health concern worldwide, with increased prevalence of overweight/obesity, up to $17 \%$ of European adults being obese (OCED/EU 2016). Strategies to lose weight mostly relay on caloric restriction and increased physical activity, which not always are easily adhered to by overweight/obese subjects and are of dubious long-term efficiency. Nutraceuticals and functional foods might be an alternative, coadjutant strategy to help lose weight and reduce associated cardiometabolic risk factors in affected subjects. There are different nutraceuticals/functional foods containing dietary fibre (DF) or plant extracts rich in polyphenols (PP), but to our knowledge, there is no nutraceutical combining both bioactive compounds. Objectives: The objective of the present study was to assess the efficiency on different risk makers of a dietary supplement combining a soluble DF (oat beta-glucan -BG-) and a green coffee bean extract (GCBE) rich in PP in overweight/obese subject. Methods: A randomized, parallel, blind, doseresponse study was performed in 60 overweight/obese subjects divided into four groups $(\mathrm{n}=15)$ who consumed during 6 weeks two servings per day of one of four nutraceutical formulations containing low (3 $\mathrm{g} / \mathrm{d}$ ) or high (5 g/d) doses of BG of different purity (35 \& 70\%) and a fixed amount of GCBE providing 600 $\mathrm{mg} / \mathrm{d}$ PP. Food intake, anthropometry and different cardiometabolic markers were assessed at the beginning and end of the intervention. Results: Results show reduced levels of total cholesterol ( $p=0.0001)$, LDLcholesterol $(\mathrm{p}=0.01)$, VLDL-cholesterol $(\mathrm{p}=0.014)$ and triglycerides $(\mathrm{p}=0.023)$, with the $35 \%$ BG being more effective than the $70 \%$ BG reducing lipid plasma levels. Although body weight and body mass index were not significantly modified, percentage of body fat significantly decreased after the intervention, the $70 \% \mathrm{BG}$ being more efficient than the 35\% BG. Conclusions: Combination of DF and PP seems an effective strategy to reduce cardiometabolic risk factors in overweight/obese subjects. Conflict of interests: The authors declare no conflicts of interest. Acknowledgement: Funded by AGL2015-69986-R.

36

Nutritional intervention in students of Obligatory Secondary Education based on the educational project "Mission X: Train like an astronaut"

A García-Carro ${ }^{a, b}$, D. Escobar-Toledo ${ }^{a, b}$, R. Torres-Peralta ${ }^{a, b}$, D. Escutia ${ }^{c}, D$. Cañada-López ${ }^{a, b}$, M. GonzálezGross $^{a, b, d}$

${ }^{a}$ ImFINE Research Group, Department of Health and Human Performance, Faculty of Physical Activity and Sport Sciences-INEF. Universidad Politécnica de Madrid, Spain.

${ }^{b}$ Red Española de Investigación en Ejercicio Físico y Salud en Poblaciones Especiales (EXERNET), Spain.

${ }^{\mathrm{C}}$ ESERO Spain. Eureopan Space Agency (ESA) - Parque de las Ciencias. Granada. Spain.

${ }^{\mathrm{d}}$ Biomedical Research Centre of Pathophysiology of obesity and nutrition, CIBERObn, B12/03/30038.

Carlos III Health Institute, Spain.

Background: "Mission X: Train like an Astronaut" (MX) launched by NASA and European Space Agency (ESA) in 2011 is an educational project. Astronauts were proposed as a role model of healthy lifestyle for children aged 8-12. Present in 24 countries, ImFINE is the Spanish Partner alongside ESERO Spain. To expand the project to adolescents is desirable as their physical inactivity and wrong eating habits are increasing issues. Objectives: 1 . To adapt MX to the Obligatory Secondary Education (OSE) curriculum and develop a Teaching Unit (TU) based on original resources provided. 2. To increase the educational offer in relation to nutritional education (NE). 3. To facilitate physical education (PE) teachers access to nutrition material. Methods: 1 . Analysis of the original project, objectives and resources. 2. Adaptation of project to OSE's curriculum 3. Elaboration of TU on nutritional intervention (NI) in OSE. Results: MX materials have been developed considering strategic lines within OSE curriculum: health education and STEM. An interdisciplinary approach of PE and science is proposed. Key points are hydration and caloric output related 
to PE subject. The present work increases curricular offer on healthy nutritional habits. It complements the contents of Biology on nutrients, food, elaboration of diets, caloric intake, causes and prevention of diseases related to food (obesity) and identification of organs involved in nutrition. In addition, it presents a TU on NI divided into seven lessons and proposes guidelines for its application. Conclusions: PE and science teachers have a responsibility for improving nutritional habits among students together with physical activity as healthy lifestyles related to good health. There is need of materials and educational projects for PE programs. The new NE materials will soon be available online at https://www.stem.org.uk/missionx. and http://esero.es/recursos/, and through specific teacher training courses along the country developed by ESERO Spain in collaboration with ImFINE. Conflicts of interest: None.

37

\title{
Longitudinal associations between physical activity and sedentary behaviours and dairy consumption. Results from the IDEFICS study
}

\author{
A. Santaliestra-Pasías ${ }^{a, b, c, d}$, E. González-Gill ${ }^{a, b, c, d}$, V. Pala, , T. Intemmann ${ }^{f}$ A. Hebestreit ${ }^{f}$ P. Russo ${ }^{g}$, C. Van \\ Aart $^{h}$ \\ ${ }^{a}$ GENUD (Growth, Exercise, NUtrition and Development) Research Group, Universidad de Zaragoza, Spain. \\ bInstituto Agroalimentario de Aragón (IA2). \\ 'Instituto de Investigación Sanitaria Aragón (IIS Aragón). \\ ${ }^{d}$ Centro de Investigación Biomédica en Red de Fisiopatología de la Obesidad y Nutrición (CIBERObn). \\ e Department of Research. Epidemiology and Prevention Unit. Fondazione IRCCS Istituto Nazionale dei \\ Tumori, Milan, Italy. \\ 'Leibniz Institute for Prevention Research and Epidemiology - BIPS, Bremen, Germany. Institute of \\ Statistics, University of Bremen, Bremen, Germany. \\ Institute of Food Sciences, National Research Council, Avellino, Italy. \\ ${ }^{h}$ Department of Public Health, Ghent University, Ghent, Belgium.
}

Background: The knowledge about the association of physical activity (PA) and sedentary behaviours (SB) with dairy consumption in childhood is limited. Objectives: To assess the longitudinal relationship between specific lifestyle behaviours (PA and SB) and dairy consumption in a sample of European children. Methods: In this analysis 1688 (50.8\% boys) children from the IDEFICS study were included. Two measurements, with 2 years' interval (T0 and T1), were conducted: dietary information were measured by a 24-hourdietary-recall via parental reporting. SB was also assessed at both time points. Objective estimation of PA was obtained by accelerometers. Different groups were defined according to the children's behaviour (PA and SB) over time, and international recommendations were based on the cut-offs of 2 hour/day of SB and 2 hour/day of moderate to vigorous PA. Multilevel ordinal logistic regression models were used to assess the group's association with dairy consumption (milk, yogurt and cheese), adjusted for potential confounders (sex, body mass index zscore, intervention versus control region, parental education level, dietary quality index, energy intake and the consumption by each dairy group at T0). Results: Nine groups of meeting or not both recommendations (SB and PA) were obtained. Those who meeting both lifestyle recommendations at both measurement points, had higher probability to consume more milk and yogurt and less cheese (p. Conclusions: These results suggest that European children with a healthy lifestyle regarding PA and SB over time, consumed more milk and yogurt in comparison with other children. Conflict of interests: The authors declare no conflicts of interest. 


\title{
Cross-sectional and longitudinal risk of metabolic syndrome and inflammation in European children: the IDEFICS study
}

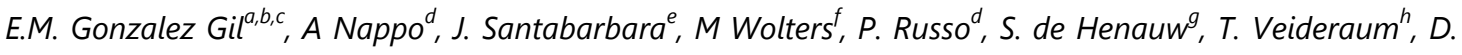 \\ Molnar ${ }^{i}$ \\ ${ }^{a}$ GENUD (Growth, Exercise, Nutrition and Development) Research Group, University of Zaragoza, \\ Zaragoza, Spain. Zaragoza. España. \\ ${ }^{b}$ Institute of Nutrition and Food Technology. Department of Biochemistry and Molecular Biology II. \\ ${ }^{c}$ Center of Biomedical Research. University of Granada. Spain. \\ dUnit of Epidemiology and Population Genetics, Institute of Food Sciences, National Research Council, \\ Avellino, Italy. \\ e Department of Preventive Medicine and Public Health, University of Zaragoza, Zaragoza, Spain. \\ fLeibniz Institute for Prevention Research and Epidemiology, Bremen, Germany. \\ ${ }^{9}$ Department of Public Health, Faculty of Medicine and Health Sciences, Ghent University,Ghent, Belgium. \\ ${ }^{h}$ National Institute for Health Development, Center of Health and Behavioral Science, Tallinn, Estonia. \\ 'Department of Pediatrics, Medical School, University of Pécs, Pécs, Hungary.
}

Background: The metabolic syndrome (MetS) has been describe as a cluster of cardiovascular risk factors. In 2014, Ahrens et al, published reference standards for a paediatric MetS score. Objectives: The aim of this study is to assess the relationship between the risk of MetS and high-sensitivity C-reactive protein (hsCRP), in a sample of European children. Methods: 2913 children (2-9 years) of the IDEFICS Study were included. Children with high density lipoprotein (HDL) cholesterol, triglycerides (TG), waist circumference (WC), diastolic and systolic blood pressure (DBP, SBP), glucose and insulin; and hs-CRP, at baseline (T0) and two years later (T1) were included. hs-CRP was categorized. Children with a MetS score $>90$ th percentile were considered at a risk level. Chi-square test was used and logistic regression adjusted by age, sex, body mass index (BMI), education level and country was performed. Results: At T0, 58\% children at risk of MetS were in the highest category of hs-CRP ( $\mathrm{p}<0.001)$ while at T1, $71.8 \%$ children at risk of MetS were in this category. Logistic regression showed that being at risk of MetS significantly increased the probability of having the highest category of hs-CRP cross-sectionally ( $\mathrm{OR}=2.07$ for $\mathrm{T} 0$ and $\mathrm{OR}=1.88$ for $\mathrm{T} 1)$. Finally, being at risk of MetS at T0 significantly increased in a $40 \%$ the probability of having the highest category of CRP in T1 $(\mathrm{OR}=1.40)$. Conclusions: Being at risk of MetS increased the probability of having an inflammatory state and this could be explained as both are risk factors that share metabolic pathways of cardiovascular diseases (CD). Improvement of the components of the MetS, already from childhood, are needed in order to prevent an inflammatory state that can lead to future CD. Conflict of interests: The authors declare no conflicts of interest.

39

\section{Menus offered in public schools of the Community of Madrid. Fitting recommendations of food groups (2007-2008)}

\author{
T. Valero-Gaspar ${ }^{a}$, A. Valenciano-Montellano ${ }^{b}$, E. Ruiz Moreno ${ }^{a}$, P. Rodríguez-Alonso ${ }^{a}$, J.M. Ávila-Torres ${ }^{a}$, G. \\ Varela-Moreiras ${ }^{a, c}$
}

aspanish Nutrition Foundation. Madrid, Spain.

bUniversity of Granada, Spain.

'Department of Pharmaceutical and Health Sciences, Faculty of Pharmacy. CEU San Pablo University. Madrid, Spain.

Introduction: School canteens must provide healthy foods for the population group they direct towards. In order to do this, dietary menu offers must achieve the standards of frequency and variety of food groups established by regional or national guides that go along with school meals programs. Objectives: To review the compliance of the recommendations by food groups included in the Technical Terms of Reference (PPT) for the school canteen service performance and to evaluate the visits carried out in the School Meals 
Programme of Madrid during the academic year 2017-2018. Methods: 34 visits were made to randomly select public centers without previous announcement. The monthly menu planning was collected and the number of portions per food group was counted, considering a full portion the main food of the dishes, as a half portion the garnish and a quarter of portion the secondary ingredients of the dishes. The recommendations were made for 20 school days. In case of fewer days, rations were adjusted to the school days of the menu's rotations. Results: 17 schools had 20-day menu's rotation, 4 schools (17 days), 12 schools (16 days), and 1 school (13 days). The average portions offered per month were: legumes (5); meat and derivatives (9); fish and seafood (6); eggs (3.5); fresh fruit (13) and dairy (4). Almost all centres (97\%) fulfilled the recommendations of legumes and $70 \%$ of fish and seafood. By contrast, $53 \%$ of schools exceeded the meat and derivatives recommendations and only $50 \%$ fulfilled the recommendations of vegetables daily. The compliance of the recommendations of eggs and fresh fruit $(32 \%$ and $29 \%$ respectively) was far below. Conclusions: According to the PPT guidelines, it is still necessary to improve the offer of food groups in menu's rotation of public schools, mainly those which are less consumed in children's diet (vegetables and fruits). Declaration of interests: The authors declare no conflict of interests

40

\title{
Data consumption on fruit juices: findings from the ANIBES Study
}

\author{
P. Rodríguez Alonso ${ }^{a}$, A. Trabanco LLano ${ }^{a}$, E. Ruiz Moreno ${ }^{a}$, T. Valero Gaspar ${ }^{a}$, J. Ibarra Morato ${ }^{a}$, J.M. Ávila \\ Torres $^{a}$, G. Varela-Moreiras ${ }^{a, b}$
}

${ }^{a}$ Spanish Nutrition Foundation (FEN). Madrid, Spain.

${ }^{b}$ Department of Pharmaceutical and Health Sciences, Faculty of Pharmacy. CEU San Pablo University. Madrid, Spain.

Background: Currently, there is a lot of controversy about the consumption of juices. In order to make appropriate recommendations, accurate data regarding preferences, consumption and contribution to the diet are needed. Objective: To know the consumption of the different juices and nectars per age groups and their energy contribution to the diet. Methods: The ANIBES Study includes a representative sample of the Spanish population between 9 and 75 years, with 2,009 participants. The random sample plus additional participants includes 2,285 participants, being representative to all age groups. The ANIBES Study consisted of a 3-day dietary recall ( 2 weekdays and 1 weekend day), fulfilled with a tablet device, where descriptions of consumed food, and photographs before and after consumption were carried out. Ingredients and grams were codified by dieticians. Results: Juices and nectars variants are consumed by $31.2 \%$ of ANIBES sample. With increasing age range, there is a reduction in the juice consumption. Regarding the type of juices, children and teenagers are the highest consumers of "juice and milk mixtures" (23.5\%, 20.4\%) followed by bottled juices $(22.1 \%, 19.9 \%)$ and nectars $(20.2 \%, 18 \%)$. Instead, home-made juices and bottled juices are more consumed by adults and elderly people (home-made juices:10.9\%, 8.3\%) (bottled juices:10.5\%, 6.3\%). This subgroup of food represents $1.3 \%$ of the daily energy intake $(2,83 \%$ in children; $2,46 \%$ in teenagers; $1.23 \%$ in adults; and $0.91 \%$ in elderly people). The average juice intake is $130.3 \mathrm{~g} / \mathrm{person} /$ day (145.8 $\mathrm{g} /$ person/day for men and $114.8 \mathrm{~g} /$ person/day for women). Conclusion: Almost one third of the population consumes some kind of juice. However, the contribution of this food group to the total energy intake is low. It is essential to conduct studies where juice categories are separated; thereby, a better diet assessment and specific recommendations can be made. Acknowledgement: The study was financially supported by CocaCola Iberia through an agreement with the Spanish Nutrition Foundation (FEN).

41

\section{Calcium and Vitamin D intake and its evolution in Spanish households according to the Food Consumption Survey (2004-2017)}

\author{
P. Rodríguez Alonso ${ }^{a}$, A. López de Subijana ${ }^{a}$, T. Valero Gaspar ${ }^{a}$, E. Ruiz Moreno ${ }^{a}$, J.M. Ávila Torres ${ }^{a}$, G. \\ Varela-Moreiras ${ }^{a, b}$ \\ aspanish Nutrition Foundation (FEN). \\ ${ }^{b}$ Department of Pharmaceutical and Health Sciences, Faculty of Pharmacy. CEU San Pablo University. \\ Madrid, Spain Madrid, Spain.
}


Background: There is a continuous need of updating dietary patterns and trends. The Food Consumption Survey (FCS) contains information about food purchases from Spanish households. Objectives: To evaluate current Calcium and Vitamin D intake and its main sources (food groups) in the Spanish diet. Methods: The data obtained from the FCS allows the estimation of the grams/person/day consumed from each and every food item. Introducing this data into the VD-FEN 2.1 software gives nutrient intake. Results: Daily Calcium intake has decreased during the assessed years (861.4 mg-2004; 807.6 mg-2011; $793.71 \mathrm{mg}$ ) in Spanish population. Instead, daily Vitamin D intake has increased (3.68 $\mu \mathrm{g}$-2004; $3.9 \mu \mathrm{g}-2011 ; 3.92 \mu \mathrm{g}-2017$ ). Daily Recommended Intake is $1000 \mathrm{mg}$ for Calcium and $15 \mu \mathrm{g}$ for Vitamin D; both current intakes are under the recommendation. In 2017, 58\% of the Calcium was obtained from dairy products, $8 \%$ from vegetables, $8 \%$ from cereals and their by-products, $5 \%$ from fruits and $4 \%$ from fish. $67 \%$ of the Vitamin D was obtained from fish, $11 \%$ from cereals and their by-products, $9 \%$ from eggs, $8 \%$ from pre-cooked meals and $2 \%$ from dairy products. The main source of Calcium was milk and dairy products. There has been a decrease in whole milk and yoghourt consumption and an increase in semi-skimmed milk, cheese and other product (especially ice-cream) consumption. Regarding the main source of vitamin D, a decrease in the fish group consumption can be observed. Conclusion: Probably calcium intake has dropped due to a descent in milk consumption. Vitamin D intake has increased, but there has been a drop in fish consumption. Both nutrient intakes are under the recommendation; there is the need to develop public health strategies in order to increase them. Conflict of interests: The authors declare no conflict of interest.

42

\title{
Adherence to Mediterranean diet and lifestyle in consumers and not-consumers of oats
}

\author{
E. Ruiz Moreno ${ }^{a}$, T.Valero Gaspar ${ }^{a}$, P. Rodríguez Alonso ${ }^{a}$, JM. Ávila Torres ${ }^{a}$, G. Varela-Moreiras $^{a, b}$ \\ ${ }^{a}$ Spanish Nutrition Foundation (FEN), Madrid, Spain. \\ ${ }^{b}$ Department of Pharmaceutical and Health Sciences, Faculty of Pharmacy, CEU San Pablo University, \\ Madrid, Spain.
}

Background: The Mediterranean Diet and lifestyle are example of healthy habits. Oats is a whole grain that would meet the characteristics food of DM. Objective: To assess Mediterranean Diet and lifestyle in consumers and not-consumers of oats. Methods: 1,832 participants were studied on through an online survey panel (CAWI System), in a random sample representative nationwide ( $>18$ years) (September 2018) of "consumers" and "non-consumers" of oats, by Nielsen area, sex and age quotas. Sample error: $\pm 2.3 \%$ for global data (95.5\% confidence interval $(\mathrm{p}=\mathrm{q}=50 \%)$.We defined "consumers" as participants who had consumed oat monthly $(\mathrm{n}=628)$ and "non-consumer" who had consumed oat annual or not eaten oats $(n=1,204)$.The Questionnaire includes 71 questions distributed into four thematic blocks: recruitment, breakfast habits, oatmeal consumption and adherence tests (MEDLIFE Test, that measures the adherence to the Mediterranean lifestyle and PREDIMED Test, that measures adherence to the Mediterranean Diet). Results: Consumers obtained higher scores in the MEDLIFE Test (score of 0-28) (13.82 \pm 2.88 ) vs nonconsumers $(12.27 \pm 3.06)$. $56.1 \%$ of consumers got 14 or more points compared to $34.8 \%$ of non-consumers. Consumers obtained higher scores in the PREDIMED Test (score of 0-7) (7.16 \pm 1.87 ) vs non-consumers $(6.03 \pm 1.85) .64 .6 \%$ of consumers got 7 or more points compared to $38.3 \%$ of non-consumers. Consumers versus non-consumers have a higher consumption of whole grains $(69.4 \%$ vs $32.9 \%)$, nuts and olives $(60.7 \%$ vs $36.5 \%)$ and fruit and natural juices (55.9\% vs $35.9 \%)$. They also reach the recommendations for physical activity (30 min/day or $150 \mathrm{~min} / \mathrm{wk}$ ) (85.2\% vs 60.0\%). Conclusion: Participants which habitually include oats in their diet have a greater adherence to the Mediterranean Diet and life style. Acknowledgements: The study was financially supported by Quaker Oats Company through an agreement with the Spanish Nutrition Foundation (FEN). 


\title{
Lyophilized Maqui-berry (Aristotelia chilensis) ameliorates hepatic steatosis in mice fed with a high fat diet.
}

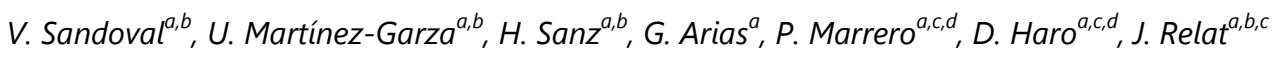 \\ ${ }^{a}$ Department of Nutrition, Food Sciences and Gastronomy. Faculty of Pharmacy and Food Science. Food \\ Campus. University of Barcelona. Barcelona-Spain. \\ ${ }^{b}$ Institute of Nutrition and Food Safety of the University of Barcelona (INSA-UB). Barcelona, Spain. \\ ${ }^{C}$ CIBER Physiopathology of Obesity and Nutrition (CIBERObn), Instituto de Salud Carlos III, E-28029 \\ Madrid, Spain. \\ ${ }^{d}$ Institute of Biomedicine of the University of Barcelona (IBUB). Barcelona, Spain.
}

Background: Hepatic steatosis is caused by an excessive accumulation of lipids in the liver. One of the main causes on the onset of hepatic steatosis is the high-fat diet (HFD)-induced obesity. The fruit of the Aristotelia chilensis commonly known as maqui-berry contains high amount of polyphenols specially anthocyanins that have shown beneficial effects on the metabolic impairment linked to obesity and its comorbidities among others. Objective: To study the effects of a lyophilized maqui-berry (LMB) in the prevention of a HFDinduced hepatic steatosis. Methods: C57BL6/J four-week-old male mice were randomized in two groups, both with a HFD but only one supplemented with LMB. The LBM was added to the beverage water and the nutritional intervention was maintained for 16 weeks. Body weight and food and drink intake were recorded every two days. Hepatic steatosis and metabolic profile were determined to define the effects of maqui supplementation. Histological analysis was performed by hematoxylin and eosin staining and gene expression analysis by qPCR. Results: Our data show that LMB reduces the body weight gain caused by HFD and the lipid droplet accumulation in the liver. Moreover,the gene expression analysis indicates that maqui supplementation changes the mRNA levels of genes involved in lipid metabolism, cholesterol homeostasis and de novo lipogenesis. Conclusion: Our evidence suggests that dietary supplementation with maqui could reduce the unhealthy effects of a HFD in the liver mainly through a reduction in the lipid accumulation and in consequence to the lipotoxicity associated to a HFD. The mechanism involved in these therapeutic effects of maqui-berry could be explained with the inhibit De Novo lipogenesis in liver. Conflict of interests: The authors declare that there are no competing interests regarding the publication of this paper. Acknowledgment: SAF2013-41093 and AGL2017-82417-R Ministry of Economy and Competitiveness, 2014SGR916 Generalitat de Catalunya, Ajut Associació Catalana de Diabetis a la recerca en diabetes 2017, National Commission of Scientific and Technological Research CONICYT-Chile, National Council of Science and Technology CONACYT-Mexico.

\section{Nutriplato ${ }^{\circledR}$, can it be a support for children's health?}

A. Marchetti ${ }^{a, b}$, E. Gesteiro $^{a, c}$, A. García-Zapico ${ }^{a, c, d}$, F. Zárate $^{e}$, M. González-Gross ${ }^{\mathrm{a}, c, f}$

amFINE Research Group, Department of Health and Human Performance, Faculty of Physical Activity and Sport Sciences-INEF. Universidad Politécnica de Madrid, Spain.

buniversità degli Studi di Milano, Milano.

${ }^{c}$ Red Española de Investigación en Ejercicio Físico y Salud en Poblaciones Especiales (EXERNET), Spain.

${ }^{d}$ Departamento de Didáctica de la Lengua, las Artes y la Educación Física, Facultad de Educación, Universidad Complutense de Madrid, Madrid, Spain.

EZaramedic CEO, Coordinador del Programa Escolar de Salud Cardiovascular (PESCA); Servicio de Pediatría Hospitales Quirónsalud Sur y Quirónsalud Toledo.

BBiomedical Research Centre of Physiopathology of obesity and nutrition, CIBERObn, B12/03/30038. Carlos III Health Institute, Spain. 


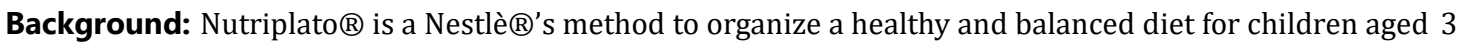
to 12. It is an adaptation inspired on the Harvard Healthy Eating Plate made for children by nutritionists from Nestlé and Sant Joan de Déu Hospital Pediatrics Nutrition Department. Objective: To evaluate if this tool can be a valid guide for both families and schools to reach and maintain an appropriate lifestyle starting from foods supply. Methods: Materials and recipes available at https://www.nutriplatonestle.es/ have been reviewed. Nutritional composition of 20 (from 30) recipes have been recalculated using the "Food Composition Database for Epidemiological Studies in Italy" (BDA). Caloric, macro and micronutrient supply have been compared with the "Dietary Reference Values (DRV) for Nutrients Summary Report" (EFSA). Results: Nutriplato ${ }^{\circledR}$ is divided into different chapters. It includes suggestions for a healthy life, tests to evaluate children's health and easy guidance to cook and serve meals according to the proportions given ( $50 \%$ vegetables and fruits, $25 \%$ healthy protein and $25 \%$ whole grains). Recipes in Nutriplato® are thought as guided examples for a balanced portion and distribution of different kinds of food in each age group (3-6 and 7-12). Macro and micronutrients supply cover a healthy daily intake from 90 to $98 \%$ of DRV considering each recipe as part of the global daily intake in 4-5 times. Conclusion: Nutriplato ${ }^{\circledR}$ can help children to get familiarized with healthier foods, understand how to balance them together and support families and schools when planning meals. Total caloric input, however, should be individually sized depending on the nutritional evaluation, according to age, gender, weight and PA level. Besides, it may be a friendly resource for pediatricians and clinics to make children's diet education. We propose to adapt Nutriplato® to adolescents aged 13 to 17 as they have different nutritional needs.

\section{Adherence to Mediterranean diet, body composition and physical fitness among Spanish adolescents}

E. Daolio ${ }^{a, b}$, A. García-Zapico ${ }^{a, c, d}$, S. Calonge-Pascual ${ }^{a, c}$, O. Gómez-Parro ${ }^{e}$, V. Martínez-Orga ${ }^{a}$, M. GonzálezGross $^{a, c, f}$, E. Gesteiro ${ }^{a, c}$

${ }^{a}$ ImFINE Research Group, Department of Health and Human Performance, Faculty of Physical Activity and Sport Sciences-INEF. Universidad Politécnica de Madrid, Spain.

bUniversità degli Studi di Milano, Milano.

${ }^{c}$ Red Española de Investigación en Ejercicio Físico y Salud en Poblaciones Especiales (EXERNET), Spain.

${ }^{d}$ Departamento de Didáctica de la Lengua, las Artes y la Educación Física, Facultad de Educación, Universidad Complutense de Madrid, Madrid, Spain.

e IES EL Pinar, Alcorcón, Madrid, Spain.

fBiomedical Research Centre of Physiopathology of obesity and nutrition, CIBERObn, B12/03/30038. Carlos III Health Institute, Spain.

Background: The nutritional transition that occurred in the last decades brought to modifications in the traditional Mediterranean Diet (MD) in Spain, towards a more western pattern, especially within the younger population. Objective: To evaluate the correlation between the adherence to the MD, body composition and physical fitness among Spanish adolescents. Methods: 124 adolescents (64 females) aged 12 to 19 attending a public secondary school in the Region of Madrid volunteered for the study. Weight and fat mass (FM) were measured by BIA (Tanita BF-350) and height with a stadiometer (Seca, $20-205 \mathrm{~cm}$ ). The KIDMED test was used to assess adherence to MD. Physical fitness was estimated with the ALPHA BATTERY. Handgrip strength was measured by dynamometry (Takey TKK 5101 Grip D), lower body muscular strength with the standing long jump test, cardiorespiratory fitness with the 20-m shuttle-run test, the agility, speed and coordination using the $4 \times 10$ test. Results: Most of adolescents (65\% of males and the $53 \%$ of females) had a medium adherence to MD, $21.7 \%$ of males and $28.1 \%$ of females had an optimal adherence and $13.3 \%$ of males and the $18.8 \%$ of females had a low adherence to MD. When analysed by age, high adherence to MD was more prevalent in those aged 12 to $14(32.1 \%)$ than in those aged 15 to 19 (19.7\%). Mean percentage of FM in males was 15.5\% (range 4.5-49\%), in females 26\% (range 7-43.8\%). Males performed better than females in all fitness tests. Higher FM was related to worse performance in all tests. Any statistical difference has been observed between adherence to MD, body composition and physical fitness in the whole group. Conclusion: Most of the adolescents showed a medium adherence to MD. Differences by sex and age could 
be observed. However, adherence to MD assessed with the KIDMED score, was not associated with body composition and physical performances. Conflict of interests: The authors declare no conflict of interest.

\title{
Eating out of home: current situation. A narrative review
}

\author{
E. García Díez ${ }^{a}$, E. Gesteiro ${ }^{a, b}$, M. González-Gross ${ }^{a, b, c, d}$ \\ ${ }^{\mathrm{a}}$ ImFine Research Group. Department of health and human performance. Universidad Politécnica de \\ Madrid. Madrid. Spain. \\ ${ }^{\text {b} C a ́ t e d r a ~ G r u p o ~ V I P S-U P M ~ d e ~ H a ́ b i t o s ~ y ~ a l i m e n t a c i o ́ n ~ s a l u d a b l e s . ~}$ \\ 'Biomedichal Research Centre of Physiopathology of obesity and nutrition (CIBERObn). Carlos III Health \\ Institute. Spain. \\ ${ }^{d}$ Red Española de Investigación en Ejercicio Físico y Salud en Poblaciones Especiales (EXERNET), Spain.
}

Background: In countries like Spain, eating out of home (EOH) is highly prevalent. Approximately, 33\% of Spanish people EOH very or quite frequently. The fact of regular EOH strongly determines the daily intakes of energy, macro and micronutrients. This turns EOH into a key element when evaluating dietary habits. Objective: To analyze what factors influence EOH and characterize the current situation. Methods: A narrative review of the scientific literature analyzing the situation of $\mathrm{EOH}$ was conducted. Literature searches were performed using the electronic database Pubmed and the search criteria was "out of home eating habits", in adults, without any geographical limit, published in the last 10 years, with full text, written in English or Spanish. Results: From 252 identified publications, only 34 met the selection criteria. They included countries in America, UE ( 3 in Spain) and Asia. A lack of consensus about the definition of EOH was observed. Most of the studies did not define EOH and those who did it, used different criteria. Most studies analyzed the relationship between $\mathrm{EOH}$ and socioeconomic, anthropometric and dietary intake variables; however, in some cases, physical activity habits, consumption places and weekdays were additionally included. Independently of the method followed, in the majority of studies an association between EOH and a higher BMI was observed; for that reason, all of them suggest interventions in order to promote healthier choices. Conclusion: This review provides data on EOH current situation. It can be a useful tool for describing the consumer profile in different countries and their preferences when EOH. Socioeconomic and anthropometric variables, along with dietary habits, influence significantly EOH, but other factors like why, where, when or how do people eat out of their homes in the general lifestyle context have to be taken into account in future researches. Conflict of interests: The authors declare no conflict of interest.

47

\section{Energy and nutrients intake analysis and performance of amateur master male athletes}

\author{
R. Torres-Peralta ${ }^{a, b}$, D. Escobar-Toledo ${ }^{a, b}$, E. García-Díez ${ }^{a}$, J. Benítez ${ }^{c}$, A. Guadalupe-Grau ${ }^{a, b}$, M. González- \\ Gross $^{a, b, d}$
}

amFINE Research Group, Department of Health and Human Performance, Faculty of Physical Activity and Sport Sciences-INEF. Universidad Politécnica de Madrid, Spain.

${ }^{\mathrm{b}}$ Red Española de Investigación en Ejercicio Físico y Salud en Poblaciones Especiales (EXERNET), Spain.

CLFE Research Group, Department of Health and Human Performance, Faculty of Physical Activity and Sport Sciences-INEF. Universidad Politécnica de Madrid, Spain.

dBiomedical Research Centre of Pathophysiology of obesity and nutrition, CIBERObn, B12/03/30038. Carlos III Health Institute, Spain.

Background. Athletes, even amateur ones, require a nutritionally balanced diet on a daily basis to maintain an adequate lifestyle and athletic performance. Periodic maximal capacity or health assessment through effort tests is recommended for master amateur athletes (MAA). Rarely are diet assessments included in these protocols. Objectives: 1 . To describe dietary intake of MAA. 2. To determine whether there is a 
relationship between nutritional patterns and maximal cardiorespiratory values $\left(\mathrm{VO}_{2} \mathrm{max}\right)$. Methods: Twenty-eight male runners (48 \pm 4.2 y.o., $174.1 \pm 6.6 \mathrm{~cm}$ height, $77.0 \pm 8.3 \mathrm{~kg}$ weight, BMI $25.2 \pm 2.0$ ) volunteered to participate in the study. Gas exchange was monitored and maximal performance was obtained during maximal incremental exercise test on a treadmill. Total body composition was assessed by DXA. Two 24-hour dietary recall interviews were done before and after maximal tests, one month apart. Meanwhile subjects continued with their habitual training program. Food and beverages consumed were analyzed for energy and nutrient content with the DIAL program. Results: Mean daily energy intake was $2611 \pm 10.1 \mathrm{Kcal}$ (median 2686; $\min 1659$, max 3326). Macronutrient contribution to energy intake for protein, carbohydrates and lipids was 18.9 $\pm 3.7 \%$ (median 18.7; min 11.2, max 27.0), 40.8 $\pm 9.1 \%$ (median 42.6; $\min 20.5$, $\max 62.0$ ) and $35.8 \pm 8.4 \%$ (median 35.7; $\min 20.0$, max 56.7), respectively. Body total Fat Mass was $20.3 \pm 5.9 \%$, Fat Free Mass $58.4 \pm 4.8 \mathrm{~kg}$, Bone Mineral Content $3.9 \pm 0.4 \mathrm{~kg}$, Bone Density $1.28 \pm 0.08$ $\mathrm{g} / \mathrm{cm}^{2}$. Mean $\mathrm{VO}_{2}$ max $54.8 \mathrm{ml} \cdot \mathrm{kg} \cdot \mathrm{min}^{-1}$, maximal speed $0.23 \mathrm{Km} \cdot \mathrm{h}^{-1} \cdot \mathrm{kg}^{-1}, 3.89 \mathrm{~W} \cdot \mathrm{kg}{ }^{-1}$. There was a negative correlation between percentage of lipid intake and $\mathrm{VO}_{2} \max (\mathrm{p}<0.05)$. Conclusions. Macronutrients contribution to energy intake differs from those recommended for athletes. Too low ingestion of carbohydrates together with too high ingestion of lipids suggests the need of nutritional intervention for this specific population of high physical activity level non-professional runners. The inverse linear correlation between percentage of lipid intake and $\mathrm{VO}_{2}$ max values supports the recommendation. Conflict of interests: The authors declare no conflict of interest.

48

\title{
PESCA project: Cardiovascular Health Program in school-aged children in Spain.
}

\author{
F. Zárate ${ }^{a}$, M. Ramón ${ }^{h}$, J. Martín de Carpi ${ }^{i}$, M. Sánchez', A. García-Zapico ${ }^{b, c, d}$, S. Aznar ${ }^{d, e}$, H. Schröder, M. \\ González-Gross ${ }^{b, g}$
}

${ }^{a}$ Zaramedic CEO, Coordinador del Programa Escolar de Salud Cardiovascular (PESCA); Servicio de Pediatría Hospitales Quirónsalud Sur y Quirónsalud Toledo.

${ }^{b}$ ImFINE Research Group, Department of Health and Human Performance, Faculty of Physical Activity and Sport Sciences-INEF. Universidad Politécnica de Madrid, Spain.

'Departamento de Didáctica de la Lengua, las Artes y la Educación Física, Facultad de Educación, Universidad Complutense de Madrid, Madrid, Spain.

${ }^{d}$ Red Española de Investigación en Ejercicio Físico y Salud en Poblaciones Especiales (EXERNET), Spain.

eGrupo de Promoción de la Actividad Física y Salud (PAFS). Facultad de Ciencias del Deporte, Universidad Castilla La Mancha.

fResearch Networking Center in Epidemiology \&Public Health (CIBERESP); IMIM-Hospital del Mar Medical Research Institute, Barcelona, Spain.

${ }^{9}$ Biomedical Research Centre of Pathophysiology of obesity and nutrition, CIBERObn, B12/03/30038.

Carlos III Health Institute, Spain.

${ }^{h}$ Cap Servei Endocrinología. Hospital Sant Joan de Déu. Barcelona.

'Jefe Servicio Gastroenterología, Hepatología y Nutrición Pediátrica. Hospital Sant Joan de Déu.

Barcelona.

${ }^{\mathrm{h}}$ Social And Health Research Center, Cuenca,Spain; School Of Education, University of Castilla-La Mancha, Ciudad Real, Spain.

Background: Recent data support that cardiovascular disease (CVD) and metabolic syndrome risk factors could already by present in overweight and obese children and adolescents. On the other hand, school has been identified as the best place to reach children for screening, prevention and intervention studies. Objectives: To develop a school-based, longitudinal 5-year study in which pediatricians and teachers collaborate in the prevention, early identification and treatment of cardiovascular (CV) risk factors in schoolaged children and adolescents. Methods: The study protocol includes annual collection of: a) Education of parents or guardians; b) Duration of pregnancy, lactation; c) family history of CVD; d) Sleep; e) Sedentarism; f) Physical activity; g) Body composition; h) Tanner stage; i) Handgrip strength; j) Cardiopulmonary auscultation; k) Weight and Height; h) Dietary habits; i) Physical fitness. FJD ethical committee has approved the study protocol. Results: A first study group has been performed in 620 subjects (aged 3 to 16) from 3 different schools at Madrid and Toledo. After informing all parents, they had to agree upon participation, so selection was not random. This may introduce some bias in the family socioeconomical background: $44 \%$ of 
mothers and $29,3 \%$ of fathers had university degree while $10 \%$ of mothers and $17,5 \%$ of fathers had only primary school studies. The designed algorithm identified $9 \%$ children at high risk, who were sent to hospital for specialized evaluation. The methodological approach seems feasible, as exploration at school did not exceed one-hour time per classroom. Conclusion: The importance of the primary care pediatricians and teacher's collaboration, specifically Physical Education teachers, has not been enough explored. PESCA study tries to fill in this gap and to develop tools and algorithms to ensure an early CV risk factors identification, in order to improve children's current and future health. Conflict of interests: None.

\section{Carob fruit extract improves the barrier integrity mucosa in the proximal colon of diabetic rats}

M. Hernández-Martín ${ }^{a}$, R. Redondo-Castillejo ${ }^{a}$, A. Macho-González ${ }^{b}$, A. Garcimartín ${ }^{a}$, M.J. González-Muñoz ${ }^{c}$ S. Bastida ${ }^{b}$, M.E. López-Oliva ${ }^{d}$

aDepartamento de Farmacología, Farmacognosia y Botánica. Facultad de Farmacia. Universidad Complutense de Madrid. Madrid. España.

${ }^{\mathrm{b}}$ Departamento de Nutrición y Ciencia de los Alimentos. Facultad de Farmacia. Universidad Complutense de Madrid. Madrid. España.

'Departamento de Ciencias Biomédicas. Unidad Docente de Toxicología. Universidad de Alcalá. Madrid. España.

${ }^{d}$ Sección Departamental de Fisiología. Facultad de Farmacia. Universidad Complutense de Madrid. Madrid. España.

Background: Type 2 Diabetes Mellitus (T2DM) is a chronic pathology characterized by hyperglycemia and insulin resistance. Alterations in the balance between the intestinal wall and the microbiota lead to secondary gastrointestinal disorders and/or chronic diseases such as T2DM. Metabolic endotoxemia, arising from the loss of barrier integrity, is thought to be a major factor that contributes to insulin resistance and T2DM. Plant polyphenols seem to have a role in the intestinal barrier regulation and several diseases prevention. Carob fruit extract (CFE) is a condensed source of polyphenols with various physiological benefits including anti-inflammatory, anti-oxidative and anti-diabetic effects. Objectives: To examine if the antioxidant capacities of CFE included in a restructured meat, as a functional food, might enhance the integrity of the barrier colonic mucosa in T2DM rats. Methods: The rats were fed a high saturated fat and cholesterol diet and treated with streptozotocin and nicotinamide to induce T2DM (65 mg/kg b.w. and 225 $\mathrm{mg} / \mathrm{kg}$ b.w., respectively). Rats were divided into two groups: DM group, diabetic with hypercholesterolemic diet and CFE-DM, diabetic with hypercholesterolemic diet and CFE. Hematoxylin-eosin (H\&E), the periodic acid-Schiff (PAS) and the Alcian blue (AB) staining were carried out on the proximal colon section according to routine methods. The immunolocalization of epithelial junction proteins, occludin and ZO-1, were evaluated by immunohistochemistry. Results: CFE supplementation increased the depth of crypts, the number of $\mathrm{AB}$ globet cells and the expression of inmunoreactivitiy of occludin along the crypts without changes in the ZO-1 immunoreactivity compared to those of the DM group. These results indicate that CFE increased the sulfomucin production and enhanced tight junction assembly by occludin, regulating the paracellular permeability and intercellular adhesion. Conclusion: CFE consumption, as a functional ingredient, through the improvement of gut barrier permeability, therefore could present an interesting strategy to treat metabolic disorders such as T2DM. Conflict of interests: The authors declare no conflict of interest.

\section{Body composition, physical activity and food intake analysis of amateur master male athletes}

L. Pantoja-Arevalo ${ }^{a}$, R. Torres-Peralta ${ }^{a, b}$, D. Escobar-Toledo ${ }^{a, b}$, M. Ugarte ${ }^{c}$, S. Calonge-Pascual ${ }^{a, b}$ GonzálezGross $M^{a, b, d}$ 
${ }^{a}$ ImFINE Research Group, Department of Health and Human Performance, Faculty of Physical Activity and Sport Sciences-INEF. Universidad Politécnica de Madrid, Spain.

${ }^{\mathrm{b}}$ Red Española de Investigación en Ejercicio Fisico y Salud en Poblaciones Especiales (EXERNET), Spain.

'Biomechanical laboratory. Faculty of Physical Activity and Sport Sciences-INEF. Universidad Politécnica de Madrid, Spain.

${ }^{d}$ Biomedical Research Centre of Pathophysiology of obesity and nutrition, CIBERObn, B12/03/30038.

Carlos III Health Institute, Spain.

Background: Athletes require a nutritionally balanced diet and non-sedentary habits in order to maintain a healthy lifestyle beyond the training sessions. Periodic health assessment through body composition analysis and diet analysis is recommended for master amateur athletes (MAA). Physical activity questionnaires are rarely part of these protocols for non-professional runners. Objectives: 1 . To describe the anthropometric profile, detailed dietary intake and total physical activity of MAA. 2. To analyze whether there is a relationship between nutritional patterns and body composition. 3. To assess relationships between energy and nutrient intake and total physical activity of this population. Methods: Twenty-eight male mature amateur runners ( $48 \pm 4.2$ y.o., $174.1 \pm 6.6 \mathrm{~cm}$ height, $77.0 \pm 8.3 \mathrm{~kg}$ weight, $25.17 \pm 2.0 \mathrm{~kg} \cdot \mathrm{m}-2$ body mass index, $\mathrm{VO}_{2} \mathrm{max} 54.8 \mathrm{ml} \cdot \mathrm{kg} \cdot \mathrm{min}^{-1}$ ) volunteered to participate in the study. Body composition was assessed by DXAs on fasting condition. Subjects answered International Physical Activity (IPAQ) and 24-hour dietary recall questionnaires. The study protocol was approved by the Ethical Committee of UPM. Results: Positive correlations between walking physical activity and total energy expenditure $(p<0.05)$; protein intake with total declared physical activity $(\mathrm{p}<0.05)$; lipids intake with moderate activity $(\mathrm{p}<0.05)$; fructose with water intake $(\mathrm{p}<0.01)$; insoluble fiber intake with lean mass $(\mathrm{p}<0.05)$. Inverse correlations were found between total lean mass and total protein intake $(\mathrm{p}<0.05)$; fructose intake with total body fat percentage $(\mathrm{p}<0.05)$; soluble fiber intake with total fat mass $(\mathrm{p}<0.05)$ and also water intake with total fat mass $(\mathrm{p}<0.05)$. Fiber intake also had a positive correlation with protein and carbohydrate intake $(p<0.05)$. Conclusion: Given the individualized approach applied to athletes regarding nutrition, it is always recommended to assess additional physical activity or body composition tests to help build a sport-specific nutritional profile.

Conflict of interests: None

51

\title{
Nutrients intake and blood biomarker status of amateur master male athletes
}

\author{
E. Gesteiro Alejos ${ }^{a, b}$, D. Escobar-Toledo ${ }^{a, b}$, S. Calonge-Pascual ${ }^{a, b}$, L. Pantoja-Arevalo ${ }^{a}$, A.E. Díaz Martínez ${ }^{a, d}$, \\ M. González-Gross ${ }^{a, b, c}$, R. Torres-Peralta ${ }^{a, b}$
}

${ }^{a}$ ImFINE Research Group, Department of Health and Human Performance, Faculty of Physical Activity and Sport Sciences-INEF. Universidad Politécnica de Madrid, Spain.

${ }^{b}$ Red Española de Investigación en Ejercicio Físico y Salud en Poblaciones Especiales (EXERNET), Spain.

'Biomedical Research Centre of Pathophysiology of obesity and nutrition, CIBERObn, B12/03/30038.

Carlos III Health Institute, Spain.

daboratorio Clínico. Centro de Medicina del Deporte. Consejo Superior de Deportes. Madrid, Spain.

Background: Amateur master athletes (MAA) is an increasing population due to physical activity promotion among adults. Energy and nutrient needs differ from those meant for the general population. Periodic health assessment through blood analysis is recommended for MAA. Seldom are detailed diet assessments included in these protocols. Objectives: 1 . To study the adequacy of some micronutrients intakes to the adequate daily intake (ADI). 2. To analyse nutritional status by blood biomarkers in MAA. Methods: Twenty-eight male runners (aged $48 \pm 4.2$, height $174.1 \pm 6.6 \mathrm{~cm}$, weight $77.0 \pm 8.3 \mathrm{~kg}$, BMI $25.2 \pm 2.0, \mathrm{VO}_{2 \mathrm{max}} 54.8 \mathrm{ml} \cdot \mathrm{kg} \cdot \mathrm{min}-1$ ) volunteered to participate in the study. Fasting blood samples were obtained to analyze basic biochemical parameters. Two 24-hour dietary recall interviews were done one month apart, subjects continued with their habitual training program. Food and beverages consumed were analyzed for energy and nutrient content with the DIALC program. Results: Mean daily energy intake $(34 \pm 7,2 \mathrm{kcal} / \mathrm{kg}$ ) was below the recommendations for athletes (45-50 kcal/kg bodyweight). Saturated fatty acids were 11,85\%E. Vitamin B12, vitamin C, vitamin E and iron intakes complied the ADI in most subjects but mean vitamin D $(6.23 \pm 9.13$ $\mu \mathrm{g}$ ) and riboflavin $(0.9 \mathrm{mg} / 1000 \mathrm{kcal})$ intakes were under recommendations and at least half the subjects were under the recommended folic acid, magnesium and zinc intakes. Thiamine $(0.78 \mathrm{mg} / 1000 \mathrm{kcal})$ and niacin $(19.8 \mathrm{mg} / 1000 \mathrm{kcal})$ intakes were higher than recommended. All the participants were under the UL 
(2000 $\mathrm{mg} / \mathrm{d})$ of calcium intake $(1063,4 \pm 363 \mathrm{mg} / \mathrm{d})$ and only half (15) ingested calcium over the recommended DRI of $1000 \mathrm{mg} / \mathrm{d}$. Biochemical and hematological parameters were in normal ranges, except for total cholesterol. Fifteen of the twenty eight volunteers had serum total cholesterol higher than 200 $\mathrm{mg} / \mathrm{dL}$. Conclusions: This population did not follow the recommended energy intake for athletes. In a general way, the studied micronutrients intake from diet was insufficient for vitamin D, calcium, folic acid, magnesium and zinc. Levels of total cholesterol in blood should be further studied in this population.

Conflict of interests: None.

52

\title{
How to get nutrition messages to the population? Testing a questionnarie
}

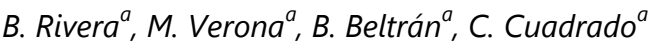

${ }^{a}$ Dpto. Nutrición y Ciencia de los Alimentos. Facultad de Farmacia. Universidad Complutense de Madrid. Madrid. Spain.

Background: Nutrition-related knowledge (NRK) and nutrition-related attitudes (NRAs) are necessary for dietary changes toward healthier dietary patterns. Objective: To know some aspects of the nutrition-related knowledge of a Spanish population. Methods: A questionnaire was developed by revising and expanding the validated survey of Parmenter K y Wradle J (2017). It included 88 items on general nutrition knowledge (e.g., dietetic recommendations, food groups, healthy decisions, health perception). For this work the first of the four sections included in this questionnaire is taken into account, 79 items were removed and the 9 referred to food were kept. Results: The $17.8 \%$ of the 45 participants were men and $82.2 \%$ were women, and they ranged in age between 18-25 years. The average of the score for first section is positive since above middle "9" of the maximum points "18". The participants achieved a higher score for the items related to the consumption of oil fish per week, consumption of alcoholic beverages per day and number of days per week that is recommended for breakfast. (Questions 5, 6 and 7) Conversely, the participants achieved a lower score for the items related to the type of milk recommended, fruit portions to the juices that are equivalent, and how much of the diet have to be provide by starch rich food. (Questions 4, 8 and 9). Conclusion: The questionnaire can be able to detect changes in nutrition knowledge over time. For this reason, it's necessary to use special emphasis in reinforcing the knowledge on items 4, 8 and 9, and to encourage to continue with the education and dissemination in the nutrition area of items 5, 6 and 7. Conflict of interests: The authors submit that there is no conflict of interest.

\section{Physical activity and Nutrition for the female athlete with polycystic ovary syndrome: An appraisal of scientific literature}

M. Pérez-Juana Cortes ${ }^{a}$, S. Calonge-Pascual ${ }^{b}$; F.M. Tobal ${ }^{a}$

${ }^{a}$ Department of Radiology, Rehabilitation, and Physiotherapy. Faculty of Medicine. Universidad Complutense de Madrid-UCM. Madrid, Spain.

${ }^{b}$ ImFINE Research Group, Department of Health and Human Performance, Faculty of Physical Activity and Sport Sciences-INEF. Universidad Politécnica de Madrid, Spain.

Background: Polycystic ovarian syndrome (PCOS) is an endocrinopathy that affects $5-13 \%$ of women in fertil age. Women with PCOS can improve if they change their lifestyle. Physical activity and nutrition are very important for this disease. Objectives: To review in the scientific literature, the physical activity and nutritional patterns and the effects of Inositol and Vitamin D supplementation in the symptoms of female athlete with PCOS. Methods: A systematic review of meta-analysis, systematic reviews and narrative reviews, from 2011 to date, has been carried out consulting PubMed database. The search terms used in combination were: "Insulin Resistance", "Hyperandrogenism", "Inositol", "Vitamin D" and "Physical Activity". Inclusion criteria were: Women (>13y. to 50y.) and PCOS patients diagnostized with insulin resistance, hyperandrogenism, overweight or obesity. Only reviews in Spanish and English language were selected. 
Results: From a total of 27 articles, were 19 finally selected as well as combinations out of these terms. This review has shown that women with PCOS improve insulin sensibility, lipid profile and body fat percentage by combined resistance training with high intensity interval training (HIIT). Besides, a healthy diet with supplementation of $2 \mathrm{~g}$ inositol, combinated of myo-inositol and D-chiro-inositol, gave better results in the ovarian stimulation protocol compared only with D-chiro-inositol. Vitamin D3 supplementation improve the common deficiency of this nutrient in women with PCOS. Conclusion: PCOS is a common endocrine disorder, with complex etiology and pathophysiology. This review shows that combined HIT and resistance training with a low carbohydrate diet $(<45 \%$ total energy intake) decrease body fat and improve insulin sensitivity. Supplementation with inositol and vitamin D improves symptomatology as irregular menstrual cycle, hirsutism and depression. Conflict of interests: The authors declare the research was conducted in the absence of any commercial or financial relationships that could be construed as potential conflict of interest.

\title{
Prevalence of sarcopenic obesity in a sample of overweight or obese subjects: Screening body composition phenotypes for a precision nutrition
}

\author{
F. Rojo Fernández ${ }^{a}$, R. de Cangas Morán ${ }^{a}$, G. Nicieza Forcelledo ${ }^{b}$, D. Zamarreño Ortiz ${ }^{c}$ \\ aDpto. Investigación en Nutrición de Precisión. Centro Salud Nutricional. Gijón (Asturias), España. \\ bDpto. de Cirugía General y del Aparato Digestivo. Hospital Universitario Central de Asturias (HUCA)- \\ Fundación Hospital del Jove. Gijón (Asturias), España. \\ 'Dpto. Urgencias. Hospital de Cabueñes. Gijón (Asturias), España.
}

Introduction: Precision nutrition gathers genotype and phenotype data and is framed within 5P medicine ${ }^{1}$. Obesity is a complex multifactorial disease ${ }^{2}$, which is defined, according to Gallagher D et al as a \%FM $\geq 30$ (men) and $\geq 40 \%$ (women) $^{3}$. Sarcopenia, a SMM disorder, characterized by a decrease in the quantity/quality/strength/fitness is associated with higher morbimortality ${ }^{4}$ and is defined, according to EWGSOP, as ASMI $<7 \mathrm{~kg} / \mathrm{m}^{2}$ (men) and $<6$ (women). BIA has been accepted to estimate ASMI ${ }^{5}$. Inbody 770 is a multifrecuency, octopolar, segmental BIA device, validated against a $4 \mathrm{C}$-model to estimate $\mathrm{FM}^{6}$ and $3 \mathrm{C}$ to estimate $\mathrm{LM}^{7}$. Objectives: To evaluate the prevalence of sarcopenic obesity by means of BIA, in a sample of patients who go to a Dietitian-Nutritionist (D-N) to lose weight. Methods: Cross-sectional study using a Spanish sample of $\mathrm{n}=91$ subjects $\left(45 \pm 14\right.$ years; $\left.\mathrm{BMI}=30 \pm 4.7 \mathrm{~kg} / \mathrm{m}^{2}\right)$, made up of 66 women ( $46 \pm 14$ years; $\left.\mathrm{BMI}=29.6 \pm 4.95 \mathrm{~kg} / \mathrm{m}^{2}\right)$ and 25 men $\left(43 \pm 13\right.$ years; $\left.\mathrm{BMI}=31.1 \pm 3.6 \mathrm{~kg} / \mathrm{m}^{2}\right)$. Inclusion criteria were adults $(>18$ years old) of both sexes, measured in the first attendance. Height was measured with a stadiometer SECA 220 (Seca Gmbh \& Co. kg.) and weight, \%FM and LMra, LMla, LMrl and LMll with Inbody 770 (Inbody Ltd; Co., Korea). The ASMI was calculated as $[\mathrm{EASM}=\mathrm{LMra}+\mathrm{LMla}+\mathrm{LMrl}+\mathrm{LMIl}] /[\mathrm{T}(\mathrm{m})]^{2}$. Results: The average \%FM and ASMI were $38.95 \pm 7.71$ (men: $32.22 \pm 6.96$; women: $41.5 \pm 6.36$ ) and $7.47 \pm 1.08 \mathrm{~kg} / \mathrm{m}^{2}$ (men: $8.82 \pm 0.68$; women: $6.96 \pm 0.68$ ). The prevalence of sarcopenic obesity was $2 \%$ (whole sample) and $3 \%$ (women: $n=2 ; 53$ and 68 years). No men presented sarcopenic obesity. Conclusions: Sarcopenic obesity might be a more common finding in the population $<60$ years old than expected, regarding that the age of one of the two women is below that threshold and the sample is small. Inbody 770 discriminates the subjects with sarcopenic obesity who could benefit from a precision nutrition in that disorder $(0.4 \mathrm{~g}$ protein $/ \mathrm{kg}$ weight/meal). Conflict of interest: The authors declare that there is no conflict of interest regarding the publication of this article. 